\title{
Exploring the BWCA (Bino-Wino co-annihilation) scenario for neutralino dark matter
}

\author{
Howard Baer, ${ }^{a}$ Tadas Krupovnickas, ${ }^{b}$ Azar Mustafayev, ${ }^{a}$ Eun-Kyung Park, ${ }^{a}$ \\ Stefano Profumo ${ }^{a, c}$ and Xerxes Tata ${ }^{d}$ \\ ${ }^{a}$ Department of Physics, Florida State University Tallahassee \\ FL 32306, U.S.A. \\ ${ }^{b}$ High Energy Theory Group, Brookhaven National Laboratory \\ Upton, NY 11973, U.S.A. \\ ${ }^{c}$ Division of Physics, Mathemathics and Astronomy, California Institute of Technology \\ Mail Code 106-38, Pasadena, CA 91125, U.S.A. \\ ${ }^{d}$ Department of Physics and Astronomy, University of Hawaii \\ Honolulu, HI 96822, U.S.A. \\ E-mail: baer@hep.fsu.edu, tadas@quark.phy.bnl.gov, mazar@hep.fsu.edu, \\ epark@hep.fsu.edu, profumo@caltech.edu, tata@phys.hawaii.edu
}

ABSTRACT: In supersymmetric models with non-universal gaugino masses, it is possible to have opposite-sign $\mathrm{SU}(2)$ and $\mathrm{U}(1)$ gaugino mass terms. In these models, the gaugino eigenstates experience little mixing so that the lightest SUSY particle remains either pure bino or pure wino. The neutralino relic density can only be brought into accord with the WMAP measured value when bino-wino co-annihilation (BWCA) acts to enhance the dark matter annihilation rate. We map out parameter space regions and mass spectra which are characteristic of the BWCA scenario. Direct and indirect dark matter detection rates are shown to be typically very low. At collider experiments, the BWCA scenario is typified by a small mass gap $m_{\widetilde{Z}_{2}}-m_{\widetilde{Z}_{1}} \sim 20-80 \mathrm{GeV}$, so that tree level two body decays of $\widetilde{Z}_{2}$ are not allowed. However, in this case the second lightest neutralino has an enhanced loop decay branching fraction to photons. While the photonic neutralino decay signature looks difficult to extract at the Fermilab Tevatron, it should lead to distinctive events at the CERN LHC and at a linear $e^{+} e^{-}$collider.

Keywords: Supersymmetry Phenomenology, Hadronic Colliders, Supersymmetric Standard Model. 


\section{Contents}

1. Introduction

2. Sparticle mass spectrum in the BWCA scenario 6

3. Dark matter in the BWCA scenario 14

3.1 Neutralino relic density in the BWCA scenario: a closer look 14

3.2 Direct and indirect detection of neutralino CDM 18

4. Neutralino radiative decay in the BWCA scenario 21

5. BWCA dark matter at colliders 24

$5.1 \widetilde{Z}_{2}-\widetilde{Z}_{1}$ mass gap in BWCA scenario 24

5.2 Fermilab Tevatron 27

5.3 CERN LHC 27

5.4 Linear $e^{+} e^{-}$collider 31

6. Conclusions 35

A. Effective theories in quantum mechanics 37

\section{Introduction}

In $R$-parity conserving supergravity models a stable neutralino $\left(\widetilde{Z}_{1}\right)$ is the lightest supersymmetric particle (LSP) over a large part of the parameter space of the model. A neutralino LSP is generally considered an excellent candidate to comprise the bulk of the cold dark matter (CDM) in the universe. The relic density of neutralinos in supersymmetric models can be calculated by solving the Boltzmann equation for the neutralino number density [1]. The central part of the calculation is to evaluate the thermally averaged neutralino annihilation and co-annihilation cross section times velocity. The computation requires evaluating many thousands of Feynman diagrams. Several computer codes are now publicly [2, 3] available to evaluate the neutralino relic density $\Omega_{\widetilde{Z}_{1}} h^{2}$.

From its analysis of the anisotropies in the cosmic microwave background radiation, the WMAP collaboration has inferred that the CDM density of the universe is given by [4,

$$
\Omega_{C D M} h^{2}=0.113 \pm 0.009
$$


Since the dark matter could well be composed of several components, strictly speaking, the WMAP measurement only implies an upper limit on the density of any single dark matter candidate. Nevertheless, even this upper bound imposes a tight constraint on all models that contain such candidate particles, and in particular, on supersymmetric models with a conserved $R$-parity [5].

Many analyses have been recently performed in the context of the paradigm minimal supergravity model [6] (mSUGRA), which is completely specified by the parameter set,

$$
m_{0}, m_{1 / 2}, A_{0}, \tan \beta \quad \text { and } \operatorname{sign}(\mu) .
$$

The mSUGRA model assumes that the minimal supersymmetric model (MSSM) is valid between the mass scales $Q=M_{G U T}$ and $Q=M_{\text {weak }}$. A common value $m_{0}\left(m_{1 / 2}\right)\left(\left(A_{0}\right)\right)$ is assumed for all scalar mass (gaugino mass) ((trilinear soft SUSY breaking)) parameters at $Q=M_{G U T}$, and $\tan \beta$ is the ratio of vacuum expectation values of the two Higgs fields that give masses to the up and down type fermions. The magnitude of the superpotential Higgs mass term $\mu$, but not its sign, is fixed so as to reproduce the observed $Z$ boson mass. The values of couplings and other model parameters renormalized at the weak scale can be computed via renormalization group (RG) evolution from $Q=M_{G U T}$ to $Q=M_{\text {weak }}$. Once these weak scale parameters that are relevant to phenomenology are obtained, sparticle masses and mixings may be computed, and the associated relic density of neutralinos can be determined.

In most of the allowed mSUGRA parameter space, the relic density $\Omega_{\widetilde{Z}_{1}} h^{2}$ turns out to be considerably larger than the WMAP value. Consistency with WMAP thus implies that neutralinos should be able to annihilate very efficiently. In the mSUGRA model, the annihilation rate is enhanced in just the following regions of parameter space, where the sparticle masses and/or the neutralino composition assume special forms.

- The bulk region occurs at low values of $m_{0}$ and $m_{1 / 2}$ [7, 8]. In this region, neutralino annihilation is enhanced by $t$-channel exchange of relatively light sleptons. The bulk region, featured prominently in many early analyses of the relic density, has been squeezed from below by the LEP2 bound on the chargino mass $m_{\widetilde{W}_{1}}>103.5 \mathrm{GeV}$ and the measured value of the branching fraction $B(b \rightarrow s \gamma)$, and from above by the tight bound from WMAP.

- The stau co-annihilation region occurs at low $m_{0}$ for almost any $m_{1 / 2}$ value where $m_{\tilde{\tau}_{1}} \simeq m_{\widetilde{Z}_{1}}$. The staus, being charged, can annihilate rapidly so that $\tilde{\tau}_{1} \widetilde{Z}_{1}$ coannihilation processes that maintain $\widetilde{Z}_{1}$ in thermal equilibrium with $\tilde{\tau}_{1}$, serve to reduce the relic density of neutralinos [9].

- The hyperbolic branch/focus point (HB/FP) region at large $m_{0} \sim$ several $\mathrm{TeV}$, where $|\mu|$ becomes small, and neutralinos efficiently annihilate via their higgsino components [10]. This is the case of mixed higgsino dark matter (MHDM).

- The $A$-annihilation funnel occurs at large $\tan \beta$ values when $2 m_{\widetilde{Z}_{1}} \sim m_{A}$ and neutralinos can efficiently annihilate through the relatively broad $A$ and $H$ Higgs resonances [11]. 
In addition, a less prominent light Higgs $h$ annihilation corridor occurs at low $m_{1 / 2}$ [12] and a top squark co-annihilation region occurs at particular $A_{0}$ values when $m_{\tilde{t}_{1}} \simeq m_{\widetilde{Z}_{1}}[13$.

Many analyses have also been performed for gravity-mediated SUSY breaking models with non-universal soft terms. Non-universality of soft SUSY breaking (SSB) scalar masses can, 1. pull one or more scalar masses to low values so that "bulk" annihilation via $t$-channel exchange of light scalars can occur [14, 15], 2. they can bring in new near degeneracies of various sparticles with the $\widetilde{Z}_{1}$ so that new co-annihilation regions open up [16, 15, 17, 3 . bring the value of $m_{A}$ into accord with $2 m_{\widetilde{Z}_{1}}$ so that funnel annihilation can occur [18, 15], or 4 . they can pull the value of $\mu$ down so that higgsino annihilation can occur [18, 19, 15]. It is worth noting that these general mechanisms for increasing the neutralino annihilation rate can all occur in the mSUGRA model. Moreover, in all these cases the lightest neutralino is either bino-like, or a bino-higgsino mixture.

If non-universal gaugino masses are allowed, then qualitatively new possibilities arise that are not realized in the mSUGRA model 20-23]. One case, that of mixed wino dark matter (MWDM), has been addressed in a previous paper [24]. In this case, as the weak scale value of $\mathrm{SU}(2)$ gaugino mass $M_{2}$ (weak) is lowered from its mSUGRA value, keeping the hypercharge gaugino mass $M_{1}$ (weak) fixed, the wino component of $\widetilde{Z}_{1}$ continuously increases until it becomes dominant when $M_{2}$ (weak) $<M_{1}$ (weak) (assuming $|\mu|$ is large). The $\widetilde{Z}_{1} \widetilde{W}_{1,2} W$ coupling becomes large when $\widetilde{Z}_{1}$ becomes wino-like, resulting in enhanced $\widetilde{Z}_{1} \widetilde{Z}_{1} \rightarrow W^{+} W^{-}$annihilations. Moreover, co-annihilations with the lightest chargino and with the next-to-lightest neutralino help to further suppress the LSP thermal relic abundance. Indeed, if the wino component of the neutralino is too large, this annihilation rate is very big and the neutralino relic density falls well below the WMAP value.

A qualitatively different case arises in supersymmetric models if the SSB gaugino masses $M_{1}$ and $M_{2}$ are of opposite sign. ${ }^{1}$ As we will see below, the transition from a binolike $\widetilde{Z}_{1}$ to a wino-like $\widetilde{Z}_{1}$ is much more abrupt as $-M_{2}$ (weak) passes through $M_{1}$ (weak). Opposite sign masses for $\mathrm{SU}(3)$ relative to $\mathrm{SU}(2)$ and $\mathrm{U}(1)$ gaugino mass parameters are well known to arise in the anomaly-mediated SUSY breaking (AMSB) model [25]. An opposite sign between bino and wino masses, which is of interest to us here, can arise in supersymmetric models with a non-minimal gauge kinetic function (GKF). In supergravity Grand Unified Theories (GUT), the GKF $f_{A B}$ must transform as the symmetric product of two adjoints of the GUT group. In minimal supergravity, the GKF transforms as a singlet. In SU(5) SUGRA-GUT models, it can also transform as a 24, 75 or 200 dimensional representation [26], while in $\mathrm{SO}(10)$ models it can transform as 1, 54, 210 and 770 dimensional representations [27, 28. Each of these non-singlet cases leads to unique predictions for the ratios of GUT scale gaugino masses, though of course (less predictive) combinations are also possible. The GUT scale and weak scale ratios of gaugino masses are listed in table 1 for these non-singlet representations of the GKF. If the GKF transforms as a linear combination of these higher dimensional representations, then essentially arbitrary gaugino masses are allowed. In this report, we will adopt a phenomenological approach as in ref. [24], and regard the three MSSM gaugino masses as independent parameters, with

\footnotetext{
${ }^{1}$ The sign of the gaugino mass under RG evolution is preserved at the one loop level.
} 


\begin{tabular}{|c|c|ccc|ccc|}
\hline \multicolumn{2}{|c|}{} & \multicolumn{3}{|c|}{$M_{\mathrm{GUT}}$} & \multicolumn{3}{c|}{$M_{Z}$} \\
\hline group & $F_{h}$ & $M_{3}$ & $M_{2}$ & $M_{1}$ & $M_{3}$ & $M_{2}$ & $M_{1}$ \\
\hline $\mathrm{SU}(5)$ & $\mathbf{1}$ & 1 & 1 & 1 & $\sim 6$ & $\sim 2$ & $\sim 1$ \\
$\mathrm{SU}(5)$ & $\mathbf{2 4}$ & 2 & -3 & -1 & $\sim 12$ & $\sim-6$ & $\sim-1$ \\
$\mathrm{SU}(5)$ & $\mathbf{7 5}$ & 1 & 3 & -5 & $\sim 6$ & $\sim 6$ & $\sim-5$ \\
$\mathrm{SU}(5)$ & $\mathbf{2 0 0}$ & 1 & 2 & 10 & $\sim 6$ & $\sim 4$ & $\sim 10$ \\
$\mathrm{SO}(10) \rightarrow G_{442}$ & $\mathbf{5 4}$ & 1 & -1.5 & -1 & $\sim 3$ & $\sim-1.3$ & $\sim-1$ \\
$\mathrm{SO}(10) \rightarrow \mathrm{SU}(2) \times \mathrm{SO}(7)$ & $\mathbf{5 4}$ & 1 & $-7 / 3$ & 1 & $\sim 3$ & $\sim-2.1$ & $\sim 0.42$ \\
$\mathrm{SO}(10) \rightarrow H_{51}$ & $\mathbf{2 1 0}$ & 1 & 1 & $-96 / 25$ & $\sim 3$ & $\sim 0.88$ & $\sim-1.6$ \\
\hline
\end{tabular}

Table 1: Relative gaugino mass parameters at $Q=M_{\mathrm{GUT}}$ and their relative values evolved to $Q=M_{Z}$ in various possible $F_{h}$ irreducible representations in SU(5) and SO(10) SUSY GUTs with a non-minimal GKF. Here, $G_{442}=\mathrm{SU}(4) \times \mathrm{SU}(2) \times \mathrm{SU}(2)$, and $H_{51}$ denotes the flipped $\mathrm{SU}(5) \times \mathrm{U}(1)$ symmetry group.

the constraint that the neutralino relic density should match the WMAP measured value. However, in this paper, we will mainly address the special features that arise when the $\mathrm{SU}(2)$ and $\mathrm{U}(1)$ gaugino masses have opposite sign.

Much work has already been done on evaluating the relic density in models with gaugino mass non-universality. Prospects for direct and indirect detection of DM have also been studied. Griest and Roszkowski first pointed out that a wide range of relic density values could be obtained by abandoning gaugino mass universality [29]. A specific form of gaugino mass non-universality occurs in AMSB models mentioned above, where the gaugino masses are proportional to the $\beta$-functions of the corresponding low energy gauge groups: $M_{1}: M_{2}: M_{3} \sim 3: 1:-10$. In this case the $\widetilde{Z}_{1}$ is almost a pure wino and so can annihilate very efficiently, resulting in a very low thermal relic density of neutralinos. This led Moroi and Randall [30] to suggest that the decay of heavy moduli to wino-like neutralinos in the early universe could account for the observed dark matter density. Corsetti and Nath investigated dark matter relic density and detection rates in models with non-minimal SU(5) GKF and also in O-II string models [31]. Birkedal-Hanson and Nelson showed that a GUT scale ratio $M_{1} / M_{2} \sim 1.5$ would bring the relic density into accord with the measured CDM density via MWDM, and also presented direct detection rates 32]. Bertin, Nezri and Orloff studied the variation of relic density and the enhancements in direct and indirect DM detection rates as non-universal gaugino masses are varied [33]. Bottino et al. performed scans over independent weak scale parameters to show variation in indirect DM detection rates, and noted that neutralinos as low as $6 \mathrm{GeV}$ are allowed [34. Belanger et al. have recently presented relic density plots in the $m_{0} v s . m_{1 / 2}$ plane for a variety of universal and non-universal gaugino mass scenarios, and showed that large swaths of parameter space open up when the $\mathrm{SU}(3)$ gaugino mass $M_{3}$ becomes small [35]: this is primarily because the value of $|\mu|$ reduces with the corresponding value of $M_{3}$, resulting in an increased higgsino content of the neutralino. Mambrini and Muñoz, and also Cerdeno and Muñoz, examined direct and indirect detection rates for models with scalar and gaugino mass nonuniversality [36]. Auto et al., auto proposed non-universal gaugino masses to reconcile the predicted relic density in models with Yukawa coupling unification with the WMAP result. 
Masiero, Profumo and Ullio exhibit the relic density and direct and indirect detection rates in split supersymmetry where $M_{1}, M_{2}$ and $\mu$ are taken as independent weak scale parameters with ultra-heavy squarks and sleptons [37.

The main purpose of this paper is to examine the phenomenology of SUSY models with non-universal gaugino masses and examine their impact upon the cosmological relic density of DM and its prospects for detection in direct and indirect detection experiments, and finally for direct detection of sparticles at the Fermilab Tevatron, the CERN LHC and at the future international $e^{+} e^{-}$linear collider (ILC). Towards this end, we will adopt a model with GUT scale parameters including universal scalar masses, but with independent $\mathrm{SU}(2)$ and U(1) gaugino masses which can be of opposite-sign. Indeed while some of the earlier studies with non-universal gaugino masses mentioned in the previous paragraph do allow for negative values of $M_{1} / M_{2}$, we are not aware of a systematic exploration of this part of parameter space. For the most part, we adjust the gaugino masses until the relic density matches the central value determined by WMAP. Whereas the case of same-sign gaugino masses allows consistency with WMAP via both bino-wino mixing and bino-wino co-annihilations (the MWDM scenario), the opposite sign case admits essentially no mixing between the bino and wino gaugino components. Agreement with the WMAP value can be attained if the LSP is bino-like, and the wino mass $M_{2} \simeq-M_{1}$ at the weak scale, so that bino-wino co-annihilation (BWCA) processes come into play and act to reduce the bino relic density to acceptable values.

The BWCA scenario leads to a number of distinct phenomenological consequences. For direct and indirect DM search experiments (except when sfermions are also very light), very low detection rates are expected in the BWCA scenario because $\mathrm{SU}(2)_{L} \times \mathrm{U}(1)_{Y}$ gauge invariance precludes couplings of the bino to gauge bosons. Regarding collider searches, the BWCA scenario yields relatively low $\widetilde{Z}_{2}-\widetilde{Z}_{1}$ mass gaps, so that two-body tree level neutralino decays are not kinematically allowed. However, when $m_{0} \lesssim .5-1 \mathrm{TeV}$, the loop-induced radiative decay $\widetilde{Z}_{2} \rightarrow \widetilde{Z}_{1} \gamma$ is enhanced, and can even be the dominant $\widetilde{Z}_{2}$ decay mode. This may give rise to unique signatures involving isolated photon plus jet(s) plus lepton(s) plus $E_{T}^{\text {miss }}$ events at the Fermilab Tevatron and the CERN LHC hadron colliders. At the ILC, $\widetilde{Z}_{1} \widetilde{Z}_{2}$ production can lead to "photon plus nothing" events, while $\widetilde{Z}_{2} \widetilde{Z}_{2}$ production can lead to diphoton plus missing energy events at large rates. It would be interesting to examine if these signals can be separated from SM backgrounds involving neutrinos, $e^{+} e^{-} \gamma$ and multiple gamma processes where the electron and positron, or some of the photons, are lost down the beam pipe. Potentially, the energy spectrum of the signal $\gamma$ may allow for the extraction of $m_{\widetilde{Z}_{2}}$ and $m_{\widetilde{Z}_{1}}$ via photon energy spectrum endpoint measurements.

The remainder of this paper is organized as follows. In section 2, we present the parameter space for the BWCA scenario, and show the spectrum of sparticle masses which are expected to occur. We also discuss some fine points of the BWCA relic density analysis. In section 3, we show rates for direct and indirect detection of DM in the BWCA scenario. These rates are expected to be below detectable levels unless some sfermions are very light or additional annihilation mechanisms can be active. In section 4, we present expectations for the radiative neutralino decay $\widetilde{Z}_{2} \rightarrow \widetilde{Z}_{1} \gamma$ in BWCA parameter space. In section 5, we 
examine the implications of the BWCA scenario for the Fermilab Tevatron, CERN LHC and the ILC. In section 6, we present our conclusions. In the appendix we adapt the idea of integrating out heavy degrees of freedom, familiar in quantum field theory, to quantum mechanics, and use the results to obtain simplified expressions for neutralino masses in the large $|\mu|$ limit where the higgsinos can be "integrated out".

\section{Sparticle mass spectrum in the BWCA scenario}

It is well known that a pure bino LSP can annihilate rapidly enough to give the observed relic density only if scalars are sufficiently light, as for instance in the so-called bulk region of the mSUGRA model. Our goal here is to explore SUGRA models with universal values of high scale SSB scalar masses and $A$-parameters, but without the assumption of universality on gaugino masses. In models without gaugino mass universality, the annihilation rate of a bino LSP may be increased in several ways, including

1. by increasing the higgsino content of the LSP, which may be achieved by decreasing the gluino mass relative to the electroweak gaugino masses [35];

2. by increasing the wino content of the LSP, by reducing the ratio $M_{1} / M_{2}$ as in the MWDM scenario which has been the subject of many investigations [32, 24];

3. by allowing co-annihilations between highly pure bino-like and wino-like states with comparable physical masses. We dub this the bino-wino coannihilation (BWCA) scenario.

The MWDM scenario is realized when the SU(2) gaugino mass parameter $M_{2}$ (weak) $\equiv$ $M_{2}\left(M_{\text {weak }}\right)$ approaches the $\mathrm{U}(1)$ gaugino mass parameter $M_{1}$ (weak). Since the $\widetilde{Z}_{1}$ in MWDM can annihilate very efficiently into $W^{+} W^{-}$pairs via its wino component, care must be taken to ensure that this wino component is not so large that the relic density falls below its WMAP value. ${ }^{2}$ The reader can easily check that if the electroweak gaugino masses are equal at the weak scale, the photino state

$$
\tilde{\gamma} \equiv \cos \theta_{W} \tilde{B}+\sin \theta_{W} \tilde{W}
$$

is an exact mass eigenstate of the tree level neutralino mass matrix, and has a mass equal to the common weak scale gaugino mass, $M$ 38]. Since the photino can readily annihilate to $W^{+} W^{-}$pairs via chargino exchange in the $t$ channel, we would expect that it gives a relic density that is smaller than the WMAP value: in other words, the wino content of the $\widetilde{Z}_{1}$ must be smaller than $\sin \theta_{W}$ in order to obtain the WMAP value of the relic density. We will see shortly that this is indeed the case.

In the MWDM scenario, treating the gaugino-higgsino mixing entries in the neutralino mass matrix, whose scale is set by $M_{W}$, as a perturbation, the signed tree level gaugino

\footnotetext{
${ }^{2}$ We emphasize that, while a neutralino relic density smaller than the WMAP value is not excluded, in this paper we confine ourselves to scenarios that accommodate this value.
} 
masses for the case $M_{1}($ weak $)=M_{2}($ weak $)=M$ are given (to second order) by,

$$
\begin{gathered}
M \text { (photino) } \\
M+\frac{1}{2} M_{Z}^{2}\left[\frac{(\sin \beta-\cos \beta)^{2}}{M-\mu}+\frac{(\sin \beta+\cos \beta)^{2}}{M+\mu}\right] \quad \text { (zino). }
\end{gathered}
$$

If instead of exact degeneracy between $M_{1}$ (weak) and $M_{2}$ (weak), we have $M_{1}$ (weak) = $M-\delta$ and $M_{2}$ (weak) $=M+\delta$, where $\delta$ is as small or comparable to the gaugino-higgsino mixing entries, these eigenvalues change to,

$$
M+\frac{1}{2}\left[g^{2}+g^{\prime 2} \mp \xi\right] v_{\text {pert }},
$$

where

$$
v_{\text {pert }}=\frac{1}{2} \frac{M_{Z}^{2}}{g^{2}+g^{\prime 2}}\left[\frac{(\sin \beta-\cos \beta)^{2}}{M-\mu}+\frac{(\sin \beta+\cos \beta)^{2}}{M+\mu}\right]
$$

and

$$
\xi^{2}=\left(g^{2}+g^{\prime 2}\right)^{2}+4\left(g^{2}-g^{\prime 2}\right) \frac{\delta}{v_{\text {pert }}}+4 \frac{\delta^{2}}{v_{\text {pert }}^{2}} .
$$

Clearly, these eigenvalues reduce to the masses (2.1) of the photino and zino states when $\delta \rightarrow 0$.

The generic case where the differences between $M_{1}$ (weak), $M_{2}$ (weak) and $\pm \mu$ are all much larger than the gaugino-higgsino mixing entries is much simpler to treat since we do not have to worry about degeneracies as in the $M_{1}$ (weak) $\simeq M_{2}$ (weak) case that we have just discussed. In the absence of gaugino-higgsino mixing, the bino and neutral wino are the gaugino mass eigenstates. Then, again treating the gaugino-higgsino mixing entries in the neutralino mass matrix as a perturbation, we see that mixing between wino and bino states occurs only at second order in $M_{W} / \mathcal{M}$, where $\mathcal{M}$ denotes a generic mass difference between the "unperturbed" higgsino and gaugino mass eigenvalues. This is in sharp contrast to the MWDM scenario where even the "unperturbed" gaugino states are strongly mixed because of the degeneracy of the eigenvalues when $M_{1}$ (weak) $=M_{2}$ (weak). It is simple to show that the signed masses of the bino-like and wino-like neutralino eigenstates take the form,

$$
\begin{aligned}
& M_{1}+\frac{1}{2} M_{Z}^{2} \sin ^{2} \theta_{W}\left[\frac{(\sin \beta-\cos \beta)^{2}}{M_{1}-\mu}+\frac{(\sin \beta+\cos \beta)^{2}}{M_{1}+\mu}\right] \text { (bino), } \\
& M_{2}+\frac{1}{2} M_{Z}^{2} \cos ^{2} \theta_{W}\left[\frac{(\sin \beta-\cos \beta)^{2}}{M_{2}-\mu}+\frac{(\sin \beta+\cos \beta)^{2}}{M_{2}+\mu}\right] \text { (wino), }
\end{aligned}
$$

where $M_{1}$ and $M_{2}$ in (2.5) denote their values at the weak scale. Note that for the special case $M_{1}$ (weak) $=-M_{2}$ (weak), the formulae (2.5) apply even though the physical masses of the two lighter neutralinos may be very close to one another. The main purpose of our discussion of the approximate masses and mixing patterns is that these will enable us to better understand the differences in phenomenology in the MWDM scenario, where $M_{1}$ (weak) is slightly smaller than $M_{2}$ (weak), and the BWCA scenario where $M_{1}$ (weak) $\simeq$ $-M_{2}$ (weak). 
Before proceeding to explore other sparticle masses in these scenarios, we first check that the qualitative expectations for neutralino mixing patterns and relic density discussed above are indeed realized by explicit calculation. Toward this end, we adopt the subprogram Isasugra, which is a part of the Isajet v $7.72^{\prime}$ event generator program [39]. ${ }^{3}$ Isasugra allows the user to obtain sparticle masses for a wide variety of GUT scale non-universal soft SUSY breaking terms. The sparticle mass spectrum is generated using 2-loop MSSM renormalization group equations (RGE) for the evolution of all couplings and SSB parameters. An iterative approach is used to evaluate the supersymmetric spectrum. Electroweak symmetry is broken radiatively, so that the magnitude, but not the sign, of the superpotential $\mu$ parameter is determined. The RG-improved 1-loop effective potential is minimized at an optimized scale to account for the most important 2-loop effects. Full 1-loop radiative corrections are incorporated for all sparticle masses. To evaluate the neutralino relic density, we adopt the IsaRED program [3], which is based on CompHEP [40] to compute the several thousands of neutralino annihilation and co-annihilation Feynman diagrams. Relativistic thermal averaging of the cross section times velocity is performed [41]. The parameter space we consider is given by

$$
m_{0}, m_{1 / 2}, A_{0}, \tan \beta, \operatorname{sign}(\mu), M_{1} \quad \text { or } \quad M_{2},
$$

where we take either $M_{1}$ or $M_{2}$ to be free parameters (renormalized at $Q=M_{G U T}$ ), and in general not equal to $m_{1 / 2}$. When $M_{1}$ is free, we maintain $M_{2}=m_{1 / 2}$, while when $M_{2}$ is free, we maintain $M_{1}=m_{1 / 2}$.

We show our first results in figure 1, where we take $m_{0}=m_{1 / 2}=300 \mathrm{GeV}$, with $A_{0}=0, \tan \beta=10, \mu>0$ with $m_{t}=178 \mathrm{GeV}$. We plot the neutralino relic density $\Omega_{\widetilde{Z}_{1}} h^{2}$ in frame $a$ ) versus variation in the $\mathrm{U}(1)$ gaugino mass parameter $M_{1}$. For $M_{1}=300 \mathrm{GeV}$, we are in the mSUGRA case, and $\Omega_{\widetilde{Z}_{1}} h^{2}=1.3$, so that this model would be strongly excluded by the WMAP measurement. For smaller values of $M_{1}$, the bino-like neutralino becomes lighter and two dips occur in the neutralino relic density. These correspond to the cases where $2 m_{\widetilde{Z}_{1}} \simeq m_{h}$ and $M_{Z}$ as one moves towards decreasing $M_{1}$, i.e. one has either light Higgs $h$ or $Z$ resonance annihilation ${ }^{4}$. Instead, as $M_{1}$ increases past its mSUGRA value, the $\widetilde{Z}_{1}$ becomes increasing wino-like and the relic density agrees with the WMAP value at $M_{1} \sim 490 \mathrm{GeV}$, where the MWDM scenario is realized [24]. For yet larger values of $M_{1}$, the wino content of $\widetilde{Z}_{1}$ is so large that the relic density falls below the WMAP value. Turning to negative values of $M_{1}$, we see that as $M_{1}$ starts from zero and becomes increasingly negative, the $Z$ and $h$ poles are again encountered. The relic density is again much larger than the WMAP bound until it begins decreasing for $M_{1}<-400 \mathrm{GeV}$. At $M_{1} \simeq-480 \mathrm{GeV}$, the relic density is again in accord with the WMAP value, while for more negative values of $M_{1}$, the relic density is too low, so that some other form of CDM or non-thermal production of neutralinos would be needed to account for the WMAP measurement.

\footnotetext{
${ }^{3}$ Isajet $7.72^{\prime}$ is Isajet v7.72 with the subroutine SSM1LP modified to yield chargino and neutralino mass diagonalization all at a common scale $Q=\sqrt{m_{\widetilde{Z}_{1}} m_{\widetilde{Z}_{2}}}$.

${ }^{4}$ For values of $\left|M_{1}\right| \lesssim 100 \mathrm{GeV}$, we have checked that the contribution of $\Gamma\left(Z \rightarrow \widetilde{Z}_{1} \widetilde{Z}_{1}\right)$ is always below limits from LEP on the invisible width of the $Z$ boson.
} 


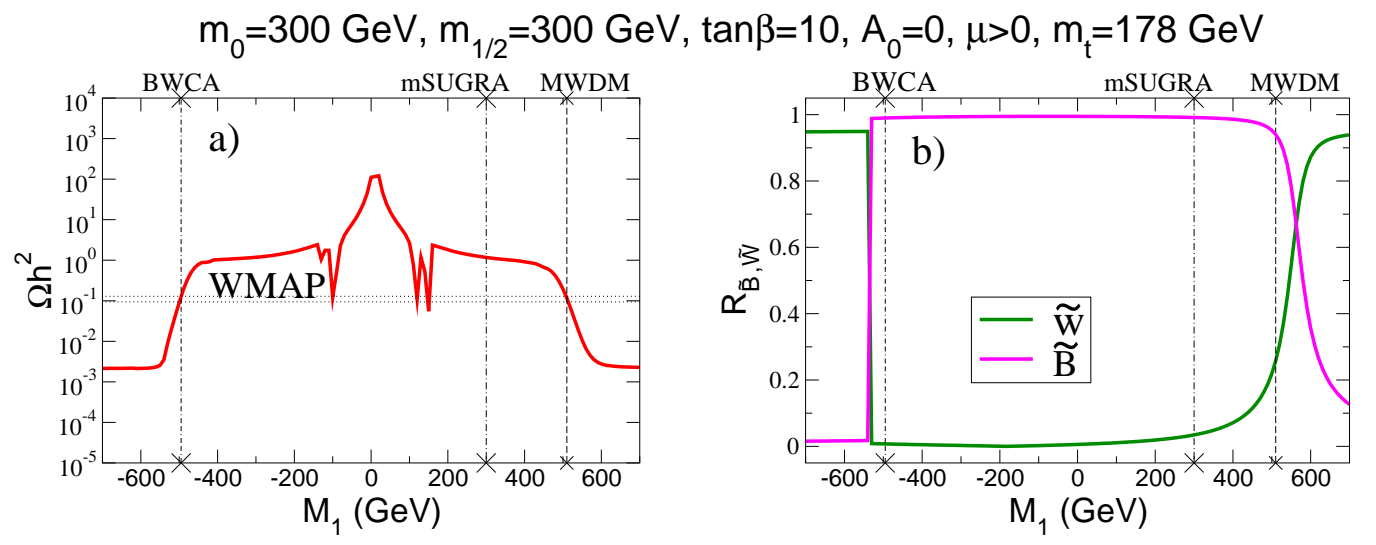

Figure 1: A plot of $a$ ) relic density $\Omega_{C D M} h^{2}$ and $b$ ) bino/wino component of the lightest neutralino as a function of $M_{1}$ for $m_{0}=300 \mathrm{GeV}, m_{1 / 2}=300 \mathrm{GeV}, A_{0}=0, \tan \beta=10, \mu>0$ and $m_{t}=$ $178 \mathrm{GeV}$.

In frame $b$ ), we show the amplitude, $R_{\widetilde{B}, \widetilde{W}}$, for the bino/wino content of the lightest neutralino $\widetilde{Z}_{1}$. Here, we adopt the notation of ref. [42, 38], wherein the lightest neutralino is written in terms of its (four component Majorana) Higgsino and gaugino components as

$$
\widetilde{Z}_{1}=v_{1}^{(1)} \psi_{h_{u}^{0}}+v_{2}^{(1)} \psi_{h_{d}^{0}}+v_{3}^{(1)} \lambda_{3}+v_{4}^{(1)} \lambda_{0},
$$

where $R_{\widetilde{W}}=\left|v_{3}^{(1)}\right|$ and $R_{\widetilde{B}}=\left|v_{4}^{(1)}\right|$. A striking difference between the positive and negative $M_{1}$ portions of frame $b$ ) is the shape of the level crossings at $R_{\widetilde{B}}=R_{\widetilde{W}}=1 / \sqrt{2}$ : while the transition from a bino-like to a wino-like LSP is gradual when $M_{1}>0$, it is much more abrupt for negative values of $M_{1}$. We had already anticipated this when we noted that for $M_{1}$ (weak) $=M_{2}$ (weak) the mass eigenstates are the photino, and aside from a small admixture of higgsinos, the zino, while the corresponding eigenstates were bino-like and wino-like as long as $\mid M_{1}$ (weak) $-M_{2}$ (weak)| was larger than the gaugino-higgsino mixing entries in the neutralino mass matrix. We also see that, for $M_{1}>0$, the MWDM scenario is realized for the value $M_{1}$ where $R_{\widetilde{W}}<\sin \theta_{W} \simeq 0.48$ as we also expected. Finally, again as we anticipated, for $M_{1}<0$, the BWCA scenario is obtained for $\left|M_{1}\right|$ just above the level crossing, when the LSP is mainly bino-like and close in mass to the wino-like $\widetilde{Z}_{1}$. For a bino-like LSP, annihilation to vector boson pairs is suppressed, and the only way to reduce the relic density in the case of large negative $M_{1}$ is by having rather small $\widetilde{Z}_{2}-\widetilde{Z}_{1}$ and $\widetilde{W}_{1}-\widetilde{Z}_{1}$ mass gaps, so that chargino and neutralino co-annihilation effects are large [43], and act to decrease the relic density. Of course, when $-M_{1}>M_{2}$, then the $\widetilde{Z}_{1}$ suddenly becomes pure wino-like, and very low relic density is obtained, as in the case of AMSB models.

Similar results are obtained by keeping $M_{1}=m_{1 / 2}$, and varying $M_{2}$, as shown in figure 2. The MWDM scenario is reached just to the right of the level crossing where $M_{2}$ (weak) is slightly larger than $M_{1}$ (weak), while the BWCA scenario is reached for $M_{2}=$ $-156 \mathrm{GeV}$, where $\mid M_{2}$ (weak) $\mid$ is again just above $\mid M_{1}$ (weak) $\mid$ and the $\widetilde{Z}_{1}$ is essentially a bino. Again, the $\widetilde{Z}_{2}-\widetilde{Z}_{1}$ and, as discussed just below, also the $\widetilde{W}_{1}-\widetilde{Z}_{1}$, mass gaps are very small, so that co-annihilation plays an essential role in reducing $\Omega_{\widetilde{Z}_{1}} h^{2}$ to an 
acceptable value. We see also that there is a region of mainly small negative $M_{2}$ values where $m_{\widetilde{W}_{1}}<m_{\widetilde{Z}_{1}}$, where a charged LSP is obtained. This is, of course, ruled out by the negative results for searches for charged stable relics from the Big Bang.

In both the MWDM and the BWCA scenarios, $\widetilde{Z}_{1}, \widetilde{Z}_{2}$ and $\widetilde{W}_{1}$ are the lightest sparticles. They will likely have a large impact upon the phenomenology of these models. Of particular interest is the ordering of the mass spectrum of these particles. We work this out in the so-called large $|\mu|$ approximation, where $|\mu| \gg M_{1}, M_{2}$ applicable in many models. The chargino sector, since it consists of just two states, is simple and $m_{\widetilde{W}_{1}}$ is given by a relatively simple and well known expression that we do not reproduce here (see e.g. ref. [38). The neutralino sector is much more complicated but, in the large $|\mu|$ approximation, it is possible to "integrate out" the higgsinos, and work with an effective low scale theory that only includes the neutral bino and wino as discussed in the appendix. The couplings of winos and binos to higgsinos in the original theory manifests itself as a mixing between winos and binos in the effective theory, where this mixing is suppressed by $1 /|\mu|$. To $\mathcal{O}\left(1 / \mu^{2}\right)$, the signed tree level neutralino masses are given (in terms of weak scale parameters) by, ${ }^{5}$

$$
\left(1+\frac{M_{W}^{2}}{\mu^{2}}\right) m_{\widetilde{Z}_{1,2}}=\frac{M_{1}+M_{2}+a \sec ^{2} \theta_{W}+M_{2} \eta \pm \kappa}{1(1+\eta)}+\mathcal{O}\left(\frac{1}{\mu^{3}}\right)
$$

where

$$
\begin{aligned}
& a=\frac{M_{W}^{2} \sin 2 \beta}{\mu} \ll\left|M_{1}\right|,\left|M_{2}\right|, \\
& \eta=\frac{M_{W}^{2}}{\mu^{2}}\left(-1+\tan ^{2} \theta_{W}\right) \ll 1,
\end{aligned}
$$

and

$$
\begin{aligned}
\kappa^{2}= & \left(M_{1}-M_{2}\right)^{2}+2 a\left(M_{1}+M_{2}\right) \sec ^{2} \theta_{W}+a^{2} \sec ^{4} \theta_{W}+ \\
& +2\left(M_{1}+M_{2}\right) M_{2} \eta-4 a\left(M_{1}+M_{2} \tan ^{2} \theta_{W}\right)-4 M_{1} M_{2} \eta .
\end{aligned}
$$

We emphasize that (2.8) is valid even when the gaugino masses are comparable to the gaugino-higgsino mixing terms in the neutralino mass matrix, and is a useful approximation as long as $|\mu|$ is large. If $M_{1}$ is not very close to $M_{2}$, we can simplify the expressions for $m_{\widetilde{Z}_{1,2}}$ by expanding $\kappa \simeq\left|M_{1}-M_{2}\right|$ plus terms that are suppressed by powers of $\mu$. Such an expansion then yields,

$$
\begin{aligned}
& m_{\widetilde{Z}_{1}}=M_{1}+\frac{M_{W}^{2}}{\mu} \tan ^{2} \theta_{W} \sin 2 \beta-M_{1} \frac{M_{W}^{2}}{\mu^{2}} \frac{\sin ^{2} 2 \beta \tan ^{2} \theta_{W}}{M_{1}-M_{2}}+\mathcal{O}\left(\frac{1}{\mu^{3}}\right), \\
& m_{\widetilde{Z}_{2}}=M_{2}+\frac{M_{W}^{2}}{\mu} \sin 2 \beta-M_{2} \frac{M_{W}^{2}}{\mu^{2}}-\frac{M_{W}^{4}}{\mu^{2}} \frac{\tan ^{2} \theta_{W}}{M_{1}-M_{2}} \sin ^{2} 2 \beta+\mathcal{O}\left(\frac{1}{\mu^{3}}\right), \\
& m_{\widetilde{W}_{1}}=M_{2}+\frac{M_{W}^{2}}{\mu} \sin 2 \beta-\frac{M_{W}^{2}}{\mu^{2}} M_{2}+\left(\frac{1}{\mu^{3}}\right),
\end{aligned}
$$

\footnotetext{
${ }^{5}$ We are abusing notation here in that we are using $m_{\widetilde{Z}_{1}}$ and $m_{\widetilde{Z}_{2}}$ to denote the signed neutralino mass, whereas everywhere else we use the same symbols to denote the physical (positive) neutralino masses.
} 

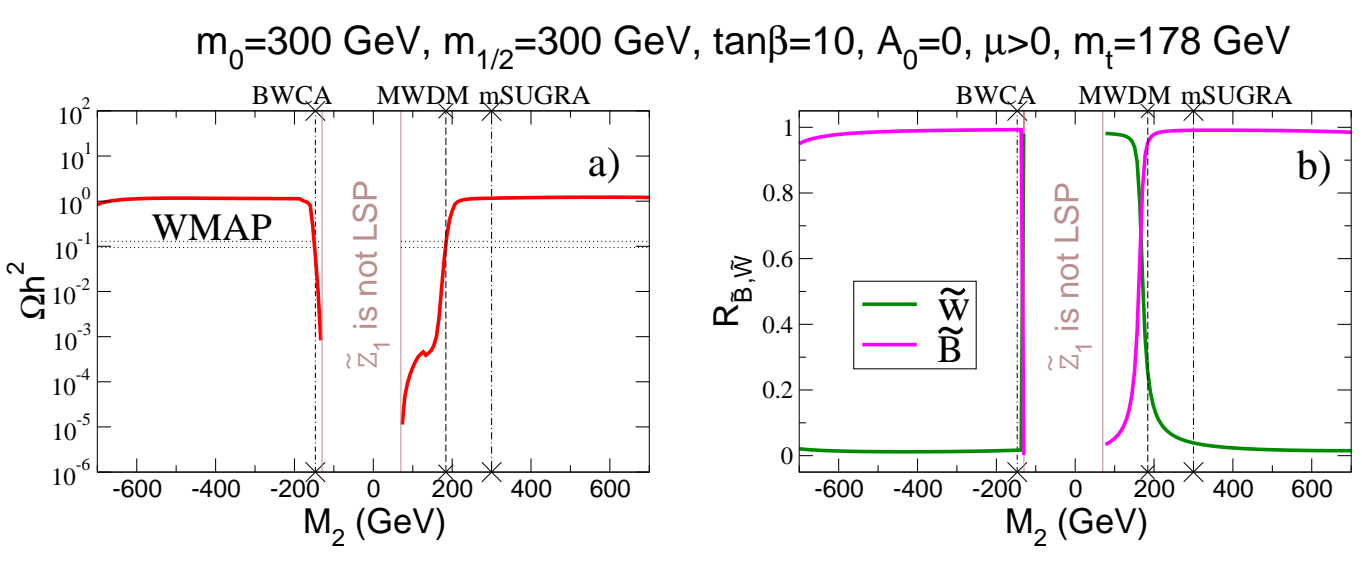

Figure 2: A plot of $a$ ) relic density $\Omega_{C D M} h^{2}$ and $b$ ) bino/wino component of the lightest neutralino as a function of $M_{2}$ for $m_{0}=300 \mathrm{GeV}, m_{1 / 2}=300 \mathrm{GeV}, A_{0}=0, \tan \beta=10, \mu>0$ and $m_{t}=178 \mathrm{GeV}$.

where we have assumed $\left|M_{1}\right|<\left|M_{2}\right|$ when we associated the bino-like state with $\widetilde{Z}_{1}$. Except for this, (2.9) are valid for all magnitudes and signs of $M_{1}$ and $M_{2}$, as long as $\left|M_{1}-M_{2}\right| \gg M_{W}^{2} /|\mu|$. In particular, we can use these expressions for the BWCA scenario, but for the MWDM case, we would have to use (2.8) to get the neutralino masses.

The ordering of the mass spectrum of the lightest SUSY particles in the same sign MWDM and opposite sign BWCA scenarios is different, as can be seen from figure 3, where we plot the $\widetilde{W}_{1}-\widetilde{Z}_{2}$ mass splitting as a function of the absolute GUT-scale value $\left|M_{1}\right| / m_{1 / 2}$. For the MWDM case, the lightest chargino is lighter than the next-to-lightest neutralino, while the opposite holds true in the BWCA case. As long as $\mid M_{1}$ (weak) $|<| M_{2}$ (weak)|, both $\widetilde{W}_{1}$ and $\widetilde{Z}_{2}$ are dominantly wino-like, and the tree-level mass splitting between them can be read off from (2.9), as long as the weak scale values of $M_{1}$ and $M_{2}$ are not too close. We then find that at tree-level [44],

$$
\Delta m_{\text {tree }} \equiv m_{\widetilde{W}_{1}}-m_{\widetilde{Z}_{2}} \approx \frac{m_{W}^{4} \tan ^{2} \theta_{W}}{\left(M_{1}(\text { weak })-M_{2}(\text { weak }) \mu^{2}\right.} \sin ^{2} 2 \beta
$$

for all combinations of signs of gaugino masses. For the BWCA case, the denominator $\mid M_{1}$ (weak) $-M_{2}$ (weak) $\mid$ is very large, so that the tree level splitting is negligible compared to the one-loop splitting; the latter is always positive, hence in the opposite sign BWCA case, when the LSP is bino-like, the lightest chargino is always heavier than the next-tolightest neutralino. In contrast, for the same-sign MWDM case, the tree level splitting (over the range of $M_{1}$ values where (2.10) is valid) is negative, and comparable to or larger than the one-loop splitting, so that the chargino is now usually heavier than $\widetilde{Z}_{2}$, as may be seen by the dashed line in figure 3 .

Various other sparticle masses are also affected by varying the gaugino masses, since these feed into the soft term evolution via the RGEs. In figure 4 , we show the variation of the sparticle mass spectrum with respect to the GUT scale ratio $M_{1} / m_{1 / 2}$ for the same parameters as in figure [1. In the mSUGRA case where $M_{1} / m_{1 / 2}=1$, there is a relatively large mass gap between $\widetilde{Z}_{2}$ and $\widetilde{Z}_{1}: m_{\widetilde{Z}_{2}}-m_{\widetilde{Z}_{1}}=106.7 \mathrm{GeV}$. As $M_{1}$ varies to large positive 


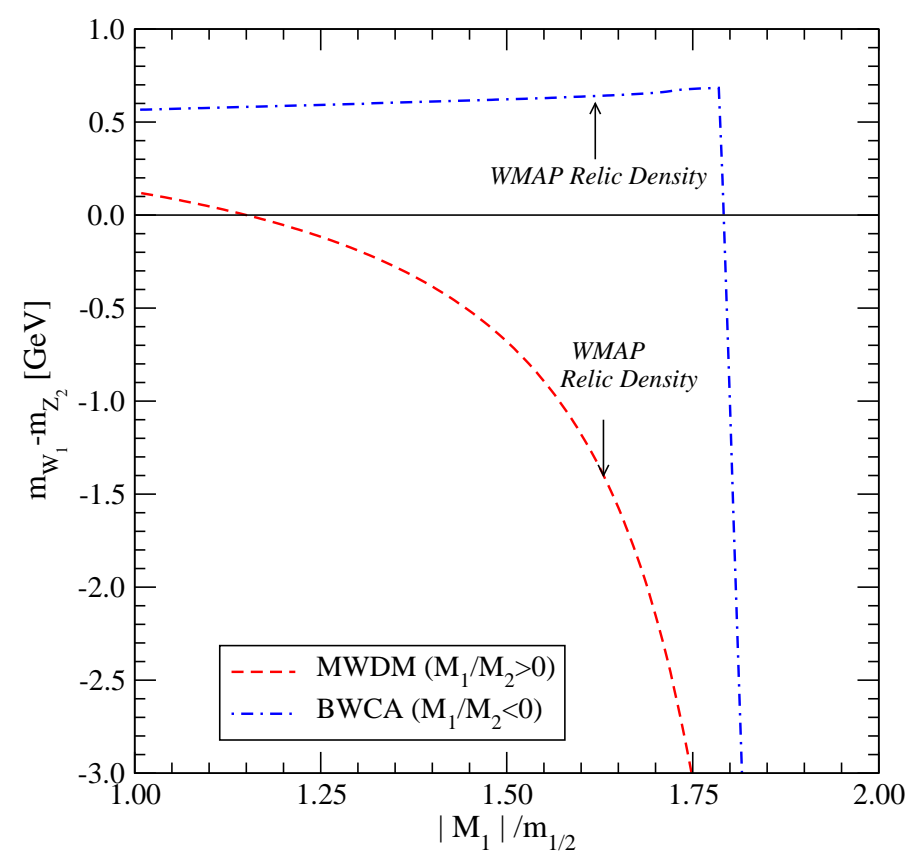

Figure 3: The mass splitting between the lightest chargino and the next-to-lightest neutralino, $m_{\widetilde{W}_{1}}-m_{\widetilde{Z}_{2}}$ in the BWCA scenario (dot-dashed blue line, with opposite $M_{1}$ and $M_{2}$ signs) and in the MWDM scenario (dashed red line, with same $M_{1}$ and $M_{2}$ signs), as a function of the absolute GUT scale value of $\left|M_{1}\right| / m_{1 / 2}$. The arrows indicate the value of $\left|M_{1}\right| / m_{1 / 2}$ where the neutralino thermal relic abundance equals the central WMAP CDM abundance value. The other SUSY parameters are as in figure $\mathrm{t}$.

values (the MWDM case), the mass gap shrinks to $m_{\widetilde{Z}_{2}}-m_{\widetilde{Z}_{1}}=31.9 \mathrm{GeV}$. As $M_{1}$ varies to large negative values, the mass gap also decreases, this time to just $22.7 \mathrm{GeV}$ in the BWCA scenario. We also note that as $\left|M_{1}\right|$ increases, the $\tilde{e}_{R}, \tilde{\mu}_{R}$ and $\tilde{\tau}_{1}$ masses also increase, since $M_{1}^{2}$ feeds into their mass evolution via RGEs. This also gives rise to the nearly symmetric behavior versus the sign of $M_{1}$ for the mass spectrum of first and second generation sfermions. As the coefficient appearing in front of $M_{1}$ in the RGEs is larger (and with the same sign) for the right handed sfermions than for the left handed ones, one expects, in general, a departure from the usual mSUGRA situation where the lightest sleptons are right-handed. As a matter of fact, whereas in mSUGRA $m_{\tilde{e}_{L}} \gg m_{\tilde{e}_{R}}$ for $m_{0} \lesssim m_{1 / 2}$, in the case of BWCA or MWDM, we find that $m_{\tilde{e}_{L}} \sim m_{\tilde{e}_{R}}$. As shown in the figure, the right-handed squark masses also increase with increasing $\left|M_{1}\right|$, although the relative effect is less dramatic than the case involving sleptons: the dominant driving term in the RGEs is, in this case, given by $M_{3}$ (absent in the case of sleptons), hence variations in the GUT value of $M_{1}$ produce milder effects. The trilinear SSB $A_{t}, A_{b}$ and $A_{\tau}$ parameters have a linear dependence on gaugino mass in their RGEs, which means the weak scale $A$-parameters will be asymmetric versus the sign of $M_{1}$. The $\mu$ parameter is also slightly asymmetric. This gives rise to the asymmetric behavior of the third generaton sfermion masses with respect to the sign of the gaugino mass.

In figure 5, we show a plot of sparticle masses for the same parameters as in figure 4, but versus $M_{2} / m_{1 / 2}$. In this case, as $\left|M_{2}\right|$ is decreased from its mSUGRA value of $300 \mathrm{GeV}$, 


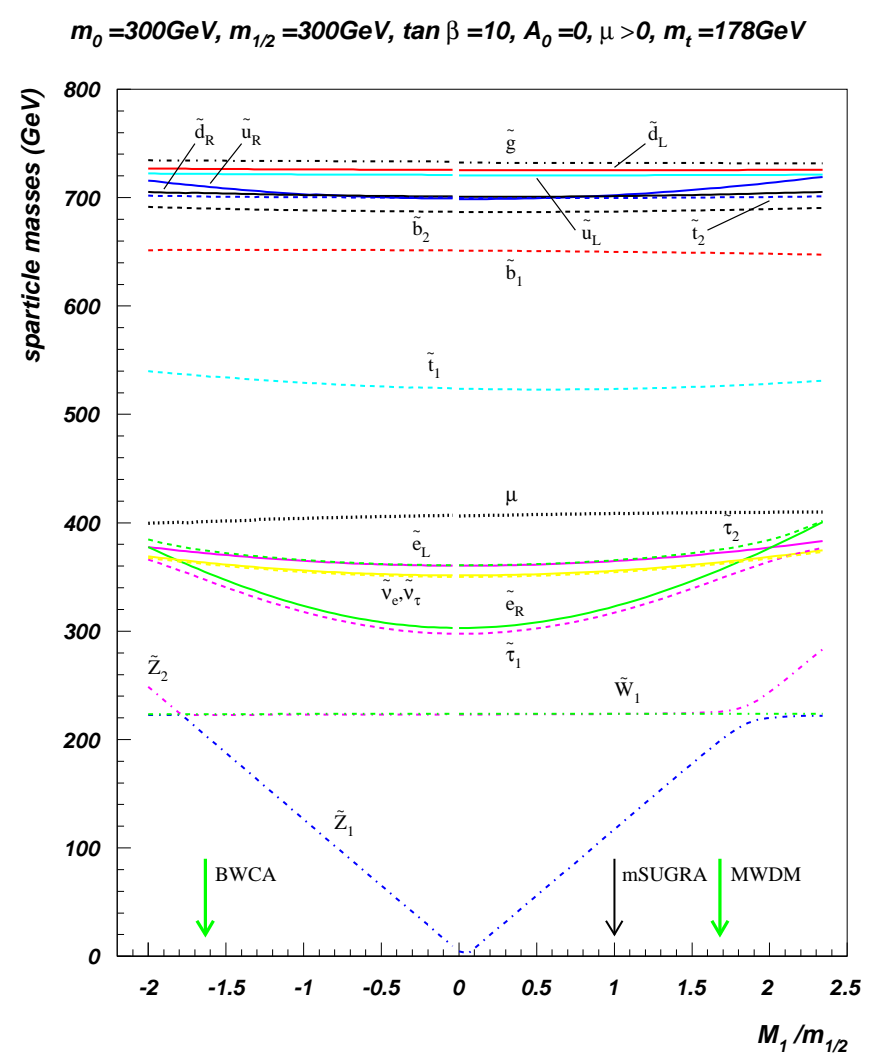

Figure 4: A plot of various sparticle masses vs. $M_{1} / m_{1 / 2}$ for $m_{0}=300 \mathrm{GeV}, m_{1 / 2}=300 \mathrm{GeV}$, $A_{0}=0, \tan \beta=10$ and $\mu>0$.

the $\widetilde{W}_{1}$ and $\widetilde{Z}_{2}$ masses decrease until $\Omega_{\widetilde{Z}_{1}} h^{2}$ reaches 0.11 in both the BWCA and MWDM scenarios. In this case, with decreasing $\left|M_{2}\right|$, the left- slepton and sneutrino masses also decrease, again leading to $m_{\tilde{e}_{L}} \sim m_{\tilde{e}_{R}}$. The left-handed squark masses similarly decrease. This increase is more pronounced that in figure 1 because the $\mathrm{SU}(2)$ gauge coupling is larger than the $\mathrm{U}(1)_{Y}$ gauge coupling. The $\mathrm{SU}(2)$ singlet right-handed sfermion masses are not affected, with the net result that the mSUGRA $m_{\tilde{e}_{L}} \gg m_{\tilde{e}_{R}}$ hierarchy is again altered.

It should be apparent now that most points in the $m_{0} v s . m_{1 / 2}$ plane can become WMAP allowed by adopting an appropriate negative value of either $M_{1}$ or $M_{2}$ such that one enters into the BWCA scenario. The exception occurs if the WMAP-allowed point is obtained because the $A$-funnel or stau co-annihilation region is reached instead. To illustrate this, we plot in figure 6 the ratio $r_{1} \equiv M_{1} / m_{1 / 2}$ in frame $a$ ) or $r_{2} \equiv M_{2} / m_{1 / 2}$ in frame $b$ ) needed to achieve a relic density in accord with the WMAP central value. We see in frame $a$ ) that $r_{1}$ generally increases as one moves from lower-left to upper-right. The structure in the upper-left of the plot occurs when $-M_{1}$ is dialed to such a value that $2 m_{\widetilde{Z}_{1}} \simeq m_{A}$, i.e. one is entering the $A$-funnel (even though $\tan \beta$ is relatively low) instead of the BWCA scenario. These regions will of course have a much larger $\widetilde{Z}_{2}-\widetilde{Z}_{1}$ mass gap than points in the BWCA scenario. Like the non-universal mass scenario [15], the BWCA scenario allows the $A$ funnel to be reached for any value of $\tan \beta$, but should be 


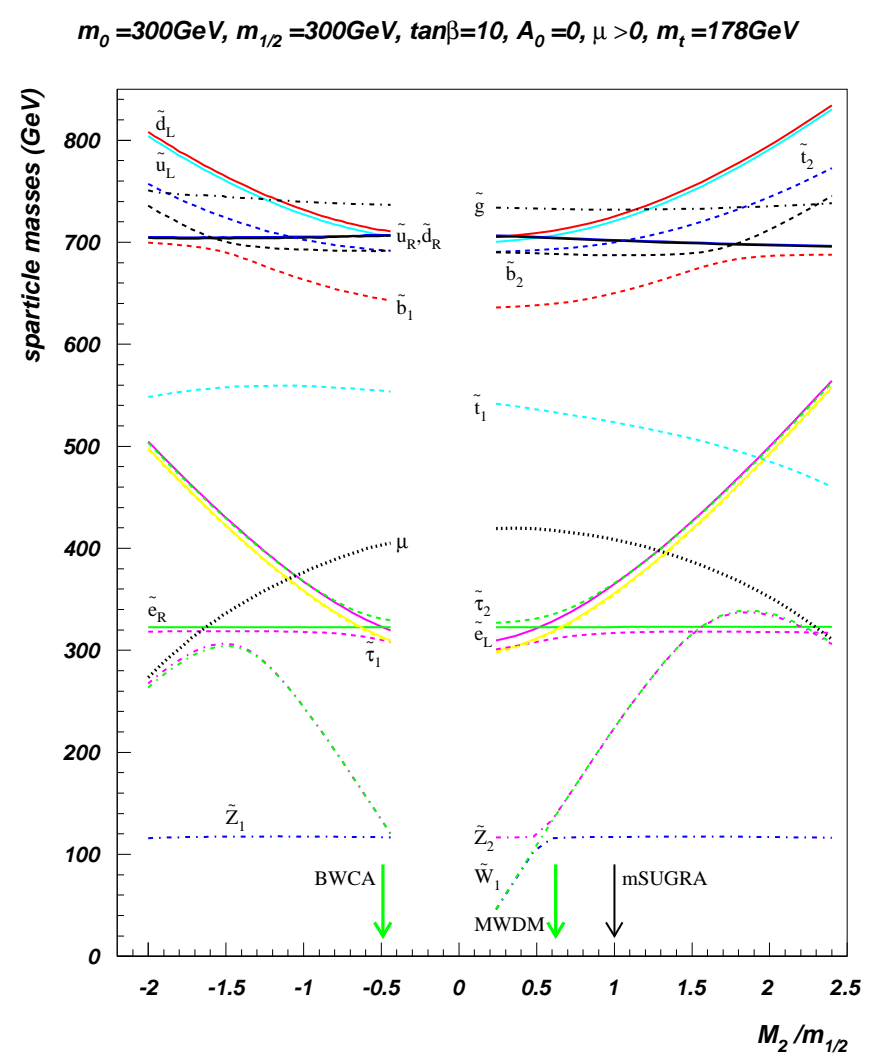

Figure 5: A plot of various sparticle masses vs. $M_{2} / m_{1 / 2}$ for $m_{0}=300 \mathrm{GeV}, m_{1 / 2}=300 \mathrm{GeV}$, $A_{0}=0, \tan \beta=10$ and $\mu>0$.

distinguishable from this because the $\widetilde{Z}_{1}-\widetilde{Z}_{2}$ mass gap, for instance, will be quite different in the two scenarios. In frame $b$ ), the ratio $r_{2}$ that gives rise to a WMAP-allowed point is shown to increase as one travels from lower to higher values of $m_{1 / 2}$. In this case, since the value of $M_{2}$ hardly changes the value of $m_{\widetilde{Z}_{1}}$, the $A$-funnel is never reached, and the BWCA region can be accessed over most of parameter space, save near the left-hand edge in the stau co-annihilation region.

\section{Dark matter in the BWCA scenario}

\subsection{Neutralino relic density in the BWCA scenario: a closer look}

In order to better understand the coannihilation mechanisms which drive the neutralino relic abundance within the WMAP preferred range in the BWCA vs. the MWDM scenario (with opposite and same signs for $M_{1}$ and $M_{2}$ ), we adopt the sample point defined by the mSUGRA input parameters $\left(m_{0}=300 \mathrm{GeV}, m_{1 / 2}=300 \mathrm{GeV}, \tan \beta=10, \operatorname{sgn}(\mu)>0, A_{0}=0\right.$, $\left.m_{\text {top }}=178 \mathrm{GeV}\right)$, and pick the $M_{1} / m_{1 / 2}$ values which give $\Omega_{\widetilde{Z}_{1}} h^{2}=0.11$, i.e., respectively, $M_{1} / m_{1 / 2}=-1.619$ and $M_{1} / m_{1 / 2}=1.630$. We plot in figure 7 the thermally averaged cross section including coannihilations (solid) and without coannihilations (dashed lines) times the relative velocity as a function of the temperature $T$. In the same sign MWDM case, 
BWCA: $\tan \beta=10, A_{0}=0, \mu>0, m_{t}=178 \mathrm{GeV}$

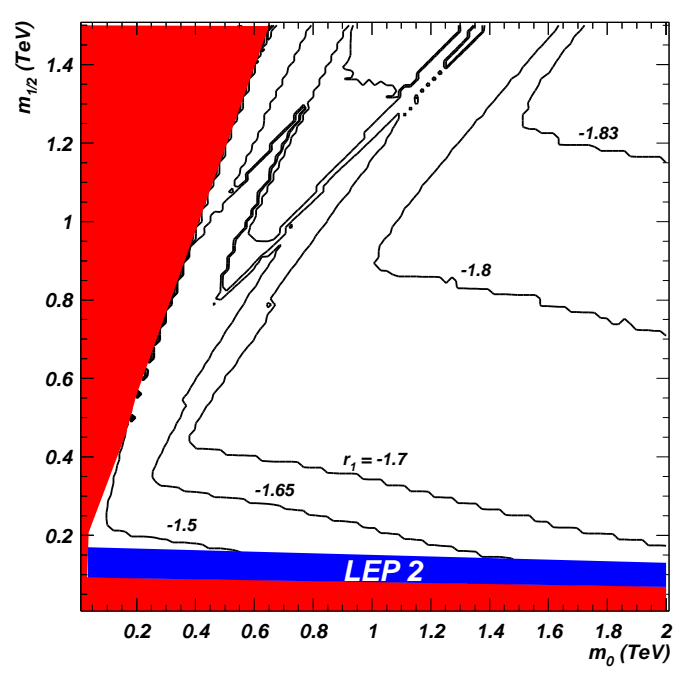

BWCA: $M_{2} \neq m_{1 / 2}, \tan \beta=10, A_{0}=0, \mu>0, m_{t}=178 \mathrm{GeV}$

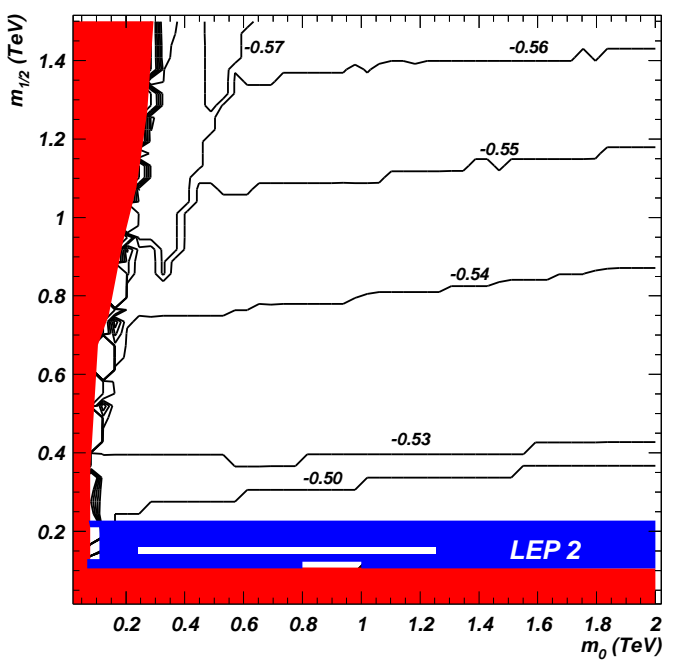

Figure 6: Contours of $a) r_{1}$ and b) $r_{2}$ in the $m_{0}$ vs. $m_{1 / 2}$ plane for $\tan \beta=10, A_{0}=0, \mu>0$. Each point has $\Omega_{\widetilde{Z}_{1}} h^{2}=0.11$. The origin of the strips in the LEP excluded region of the right hand frame is discussed in section 5 .

one notices that the very significant wino-component in the photino-like lightest neutralino gives a non-negligible $s$-wave contribution via annihilation to $W$ pairs, (i.e., a contribution which goes as $\langle\sigma v\rangle(T) \sim a$ ), while in the opposite sign BWCA case the lightest neutralino is a pure bino, and the squark mediated $t$-channel dominated pair annihilation cross section is strongly $s$-wave suppressed $\left(\langle\sigma v\rangle(T) \sim a^{\prime}+b^{\prime}\left(T / m_{\mathrm{LSP}}\right), a^{\prime} \ll b^{\prime}\right)$. As a result, the pair annihilation cross section at $T=0$ (relevant for indirect DM detection) is more than one order of magnitude suppressed in the BWCA case.

A second effect, also traced back to the lack of a significant wino component in the opposite sign BWCA case, is the role and onset of coannihilations. To isolate the role of coannihilations, we show by dashed lines $\langle\sigma v\rangle(T)$ computed without any coannihilation contribution. In the BWCA case, coannihilations play a much more important role, as can be understood by looking at the relative size of the cross sections with and without coannihilations around the lightest neutralino freeze-out temperature $T_{\text {f.o. }}$, indicated by an arrow in the figure. Furthermore, in the BWCA case, the onset of the coannihilation regime takes place at lower temperatures, a reflection of the reduced mass splitting between the coannihilation partners and the LSP. At the freeze-out temperature, $\langle\sigma v\rangle(T)$ is dominated by coannihilations in the opposite sign BWCA case, while the relative coannihilation contribution for the MWDM case (for which the $\widetilde{Z}_{1}$ s can annihilate to $W W$ ) is significantly smaller. Observe also the indicated relic abundance of the LSP computed without coannihilations, respectively 0.759 in the BWCA case and 0.138 in the same MWDM.

Coannihilations of particles $A$ and $B$ effectively enter the neutralino pair annihilation cross section when the center of mass momentum $p_{\text {c.m. }}$ of the neutralino-neutralino system satisfies the relation

$$
s=4 p_{\mathrm{c} . \mathrm{m} .}^{2}+4 m_{\widetilde{Z}_{1}}^{2} \geq\left(m_{A}+m_{B}\right)^{2}
$$




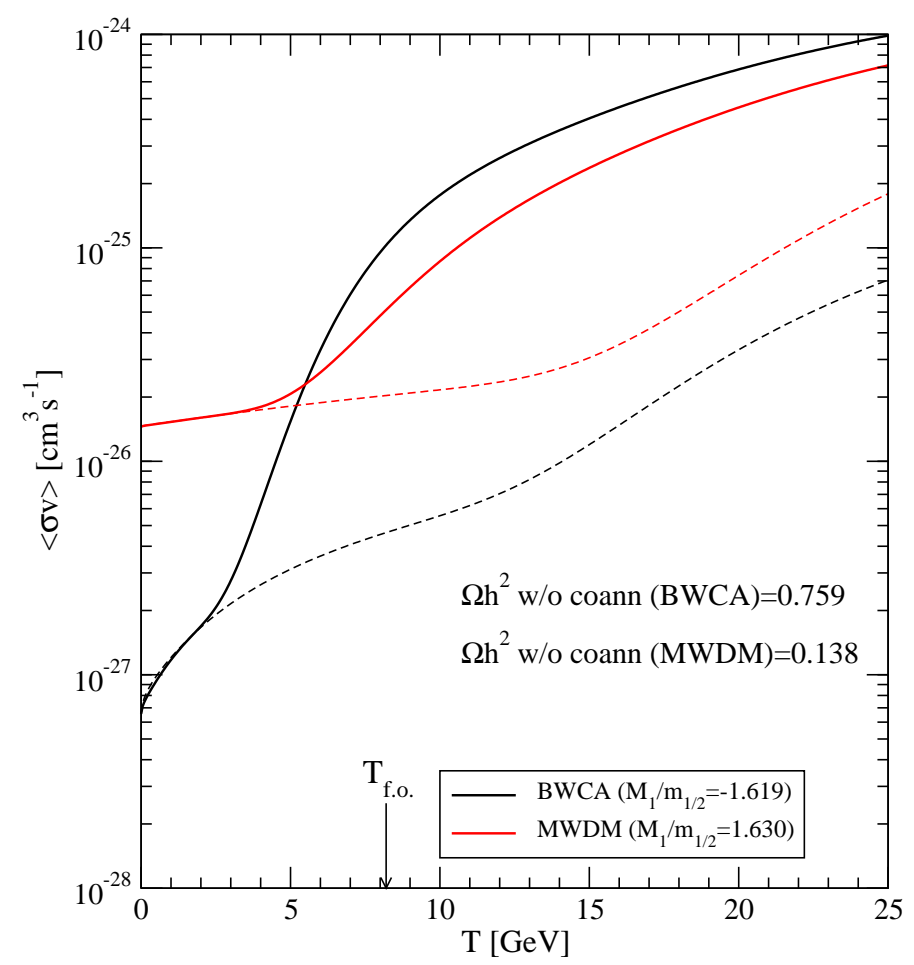

Figure 7: The thermally averaged effective pair annihilation cross section times velocity, $\langle\sigma v\rangle(T)$ as a function of the temperature, for the BWCA (black lines) and MWDM (red lines) scenarios. The dotted lines correspond to $\langle\sigma v\rangle(T)$ without the inclusion of coannihilation effects. The arrow indicates the approximate temperature where freeze-out occurs.

A suitable quantity to illustrate the onset of coannihilations is given by an effective annihilation rate $(\sigma v)_{\text {eff }}$, defined as in ref. 45

$$
(\sigma v)_{\mathrm{eff}} \equiv \frac{W_{\text {eff }}\left(p_{\text {c.m. }}\right)}{4 E_{\text {c.m. }}^{2}}, \quad E_{\text {c.m. }}=\sqrt{p_{\text {c.m. }}^{2}+m_{\widetilde{Z}_{1}}^{2}}
$$

(we refer the reader to ref. 445] for the definition of the effective annihilation rate $W_{\text {eff }}$, which is essentially the sum over all (co-)annihilation channels, properly weighted, of the various annihilation rates per unit volume and unit time) and such that

$$
\lim _{p_{\mathrm{c} . \mathrm{m} . \rightarrow 0}}(\sigma v)_{\mathrm{eff}}=\langle\sigma v\rangle(T=0) .
$$

The temperature dependence of $\langle\sigma v\rangle(T)$ is factored out in the weight function $\kappa\left(p_{\text {c.m. }}, T\right)$ 45, so that

$$
\langle\sigma v\rangle(T)=\int_{0}^{\infty} \mathrm{d} p_{\text {c.m. }} \frac{W_{\text {eff }}\left(p_{\text {c.m. }}\right)}{4 E_{\text {c.m. }}^{2}} \kappa\left(p_{\text {c.m. }}, T\right) .
$$

In figure 8, we plot the two effective annihilation rates $(\sigma v)_{\text {eff }}$ for the two BWCA and MWDM sample cases at the mSUGRA point given above, with the correct WMAP relic abundance. The coannihilation thresholds are shifted to larger $p_{\text {c.m. }}$ values in the same sign case (the mass splitting of coannihilating partners is increased). The $\widetilde{W}_{1}-\widetilde{Z}_{2}$ mass splitting is too small to resolve the separate contributions, and the two bumps correspond to the 


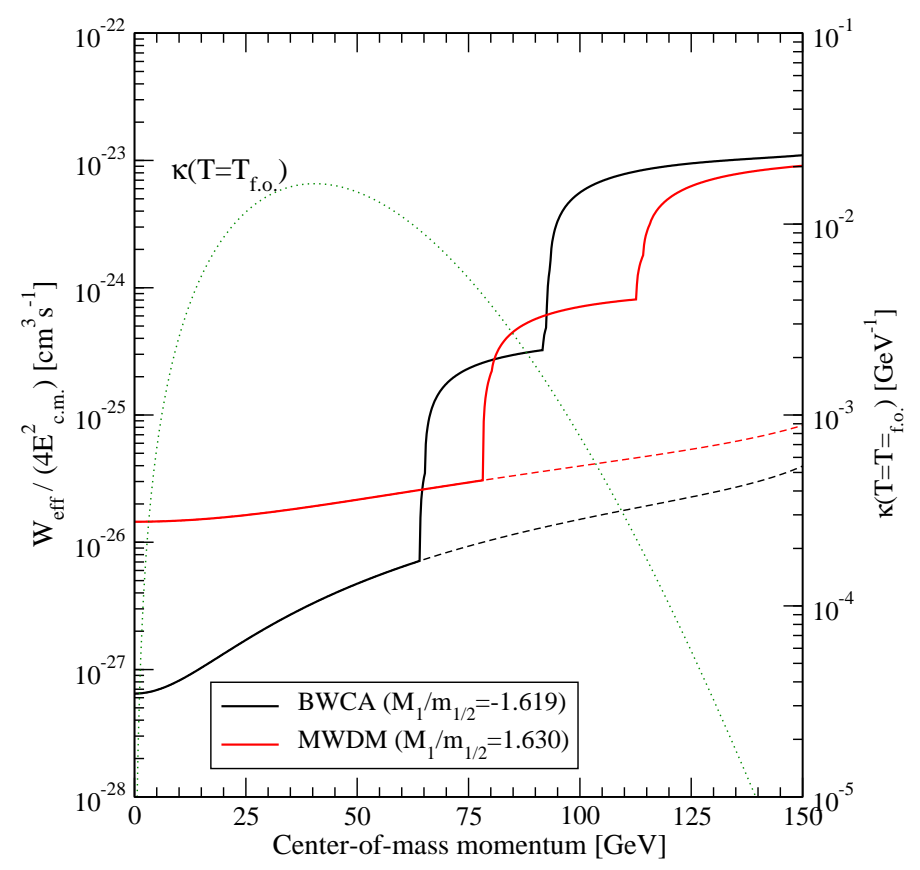

Figure 8: The effective $(\sigma v)_{\text {eff }}$ term, as defined in eq. (3.2), as a function of the center of mass momentum $p_{\text {eff }}$, for the BWCA (black lines) and MWDM (red lines) scenarios, with (solid lines) and without (dotted lines) coannihilations. The green dotted line indicates the "weight function" $\kappa\left(p_{\text {c.m. }}, T=T_{\text {f.o. }}\right)$.

onset of $\widetilde{Z}_{1}-\widetilde{W}_{1}, \widetilde{Z}_{2}$ coannihilations and the onset of (co-)annihilations amongst $\widetilde{W}_{1}$ and $\widetilde{Z}_{2}$. When the bino-wino mixing is suppressed, the second processes contribute much more than the first processes to $(\sigma v)_{\text {eff }}$. However, as shown by the weight function $\kappa\left(p_{\text {c.m. }}, T=T_{\text {f.o. }}\right)$, $(\sigma v)_{\text {eff }}$ is largely sampled in a $p_{\text {c.m. }}$. range where the $\widetilde{W}_{1}, \widetilde{Z}_{2}$ (co-)annihilations are not kinematically accessible. This picture, however, depends on the LSP mass: had we picked a larger value for $m_{\widetilde{Z}_{1}}$, the mass splitting between $\widetilde{Z}_{1}$ and $\widetilde{Z}_{2}, \widetilde{W}_{2}$ needed to obtain a sufficiently low relic abundance would have been smaller, so that the coannihilation bumps in $W_{\text {eff }} / 4 E^{2}$ would have occurred at smaller center-of-mass momenta, where the sampling function $\kappa$ has not yet fallen to very small values. In this case, the role of $\widetilde{W}_{1}-\widetilde{Z}_{2}$ coannihilations would have been enhanced even in the MWDM case.

In the limit in which sfermions are heavy, and thus the bino annihilation cross section is extremely suppressed, and in which $\mu \gg M_{1}$ (weak), $\mathrm{M}_{2}$ (weak) (in the remainder of this paragraph, we will implicitly use $M_{1,2}=M_{1,2}$ (weak)), i.e., in the pure gaugino limit, the relic abundance should only depend on $i$.) the LSP mass scale and on ii.) the LSPwino system splitting relative to the LSP mass. Since a pure wino-like system has a relic abundance which goes like 46]

$$
\Omega_{\text {pure winos }} h^{2} \simeq c \cdot\left(\frac{M_{2}}{1 \mathrm{TeV}}\right)^{\gamma}, \quad c \simeq 0.024, \gamma \simeq 1.9,
$$

the relic abundance of a coannihilating bino will be given by that of the pure wino system, rescaled by the exponential factor $\left.\exp \left[-\left(\left(M_{2}-M_{1}\right) / M_{1}\right) \cdot \widetilde{x}\right)\right]$, with $\widetilde{x} \simeq M_{1} / T_{\text {f.o. }}$, and 


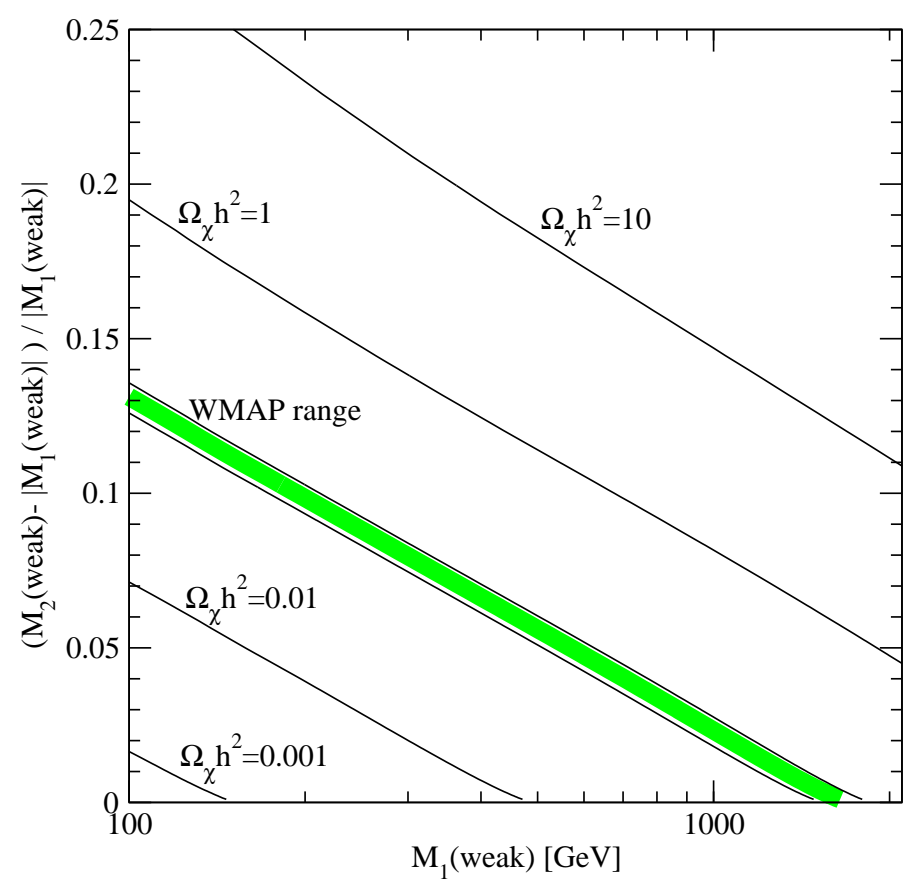

Figure 9: Iso-level curves at given values of the neutralino relic abundance in the $M_{1},\left(M_{2}-\right.$ $\left.\left|M_{1}\right|\right) /\left|M_{1}\right|$ plane. The green strip indicates the WMAP 2- $\sigma$ range. We assumed here $\mu, m_{\widetilde{S}}, m_{A}=$ $100 \cdot M_{2}, m_{\widetilde{S}}$ being all sfermion masses.

rescaled by the new parasite bino degrees of freedom ${ }^{6}$. The wino system carries $2+4$ degrees of freedom, while the bino 2 , hence we expect an enhancement of the relic abundance for a coannihilating bino of a factor $(4 / 3)^{2}$ [47. The relic abundance should then take the form

$$
\Omega_{\text {bino+wino }} h^{2} \approx a \cdot\left(\frac{M_{2}}{1 \mathrm{TeV}}\right)^{\gamma} \cdot \exp \left[\frac{M_{2}-M_{1}}{M_{1}} \cdot \widetilde{x}\right], \quad a \approx c(4 / 3)^{2} .
$$

Since $M_{2} \simeq M_{1}$, this allows in principle to define a strip in the $M_{1}, \frac{M_{2}-M_{1}}{M_{1}}$ plane of WMAP preferred relic abundance. We show the result in figure 9, taking $\mu, m_{\widetilde{S}}, m_{A}=100 \cdot M_{2}$, $m_{\widetilde{S}}$ being all sfermion masses. Indeed, the iso-level curves for the relic abundance show the functional form of eq. (3.6).

\subsection{Direct and indirect detection of neutralino CDM}

In this section, we turn to consequences of the BWCA scenario for direct and indirect detection of neutralino dark matter [48]. We adopt the DarkSUSY code [49], interfaced to Isajet, for the computation of the various rates, and resort to the Adiabatically Contracted N03 Halo model [50] for the dark matter distribution in the Milky Way. ${ }^{7}$ We evaluate the following neutralino DM detection rates:

\footnotetext{
${ }^{6}$ The degrees of freedom should be as well weighted according to the mass splitting, but this is a higher order effect.

${ }^{7}$ For a comparison of the implications of different halo model choices for indirect DM detection rates, see e.g. refs. $51-53,15$.
} 
- Direct neutralino detection via underground cryogenic detectors [54]. Here, we compute the spin independent neutralino-proton scattering cross section, and compare it to expected sensitivities [55] for Stage 2 detectors (CDMS2 [56], Edelweiss2 [57], CRESST2 [58], ZEPLIN2 [59]) and for Stage 3, ton-size detectors (XENON 60, GERDA [61, ZEPLIN4 62] and WARP 63]). We take here as benchmark experimental reaches of Stage 2 and Stage 3 detectors the projected sensitivities of, respectively, CDMS2 and XENON 1-ton at the corresponding neutralino mass.

- Indirect detection of neutralinos via neutralino annihilation to neutrinos in the core of the Sun [64. Here, we present rates for detection of $\nu_{\mu} \rightarrow \mu$ conversions at Antares [65] or IceCube [66]. The reference experimental sensitivity we use is that of IceCube, with a muon energy threshold of $25 \mathrm{GeV}$, corresponding to a flux of about 40 muons per $\mathrm{km}^{2}$ per year.

- Indirect detection of neutralinos via neutralino annihilations in the galactic center leading to gamma rays [67, as searched for by EGRET [68], and in the future by GLAST [69]. We evaluate the integrated continuum $\gamma$ ray flux above a $E_{\gamma}=1 \mathrm{GeV}$ threshold, and assume a GLAST sensitivity of $1.0 \times 10^{-10} \mathrm{~cm}^{-2} \mathrm{~s}^{-1}$.

- Indirect detection of neutralinos via neutralino annihilations in the galactic halo leading to cosmic antiparticles, including positrons [70] (HEAT [71], Pamela [72] and AMS-02 [73]), antiprotons [74] (BESS [75], Pamela, AMS-02) and anti-deuterons ( $\bar{D} \mathrm{~s})$ (BESS [76], AMS-02, GAPS [77]). For positrons and antiprotons we evaluate the averaged differential antiparticle flux in a projected energy bin centered at a kinetic energy of $20 \mathrm{GeV}$, where we expect an optimal statistics and signal-to-background ratio at space-borne antiparticle detectors [53, 78]. We take the experimental sensitivity that of the Pamela experiment after three years of data-taking as our benchmark. Finally, the average differential antideuteron flux has been computed in the $0.1<T_{\bar{D}}<0.4 \mathrm{GeV}$ range, where $T_{\bar{D}}$ stands for the antideuteron kinetic energy per nucleon, and compared to the estimated GAPS sensitivity [77] (see ref. [79] for an updated discussion of the role of antideuteron searches in DM indirect detection).

In figure 10, we show various direct and indirect DM detection rates for $m_{0}=m_{1 / 2}=$ $300 \mathrm{GeV}$, with $A_{0}=0, \tan \beta=10$ and $\mu>0$, while $M_{1}$ is allowed to vary. The $M_{1}$ value corresponding to the mSUGRA model is denoted by a dot-dashed vertical line, while the BWCA and MWDM scenarios with $\Omega_{\widetilde{Z}_{1}} h^{2}=0.11$ are denoted by dash-dash-dot and dashed vertical lines, respectively. The dotted lines correspond to the sensitivity level of each of these experiments; i.e., the signal is observable only when the model prediction is higher than the corresponding dotted line. While the minimum sensitivity for the direct detection rates in frames $b)-f$ ) refers to the minimum magnitude of the signal that is detectable (and hence independent of the LSP mass), the smallest detectable cross section shown by the dotted curves in frame $a$ ) depends on the value of $m_{\widetilde{Z}_{1}}$.

In frame $a$ ), we plot the spin-independent neutralino-proton scattering cross section. We see that as $M_{1}$ is decreased, and becomes increasingly negative, the neutralino-proton 

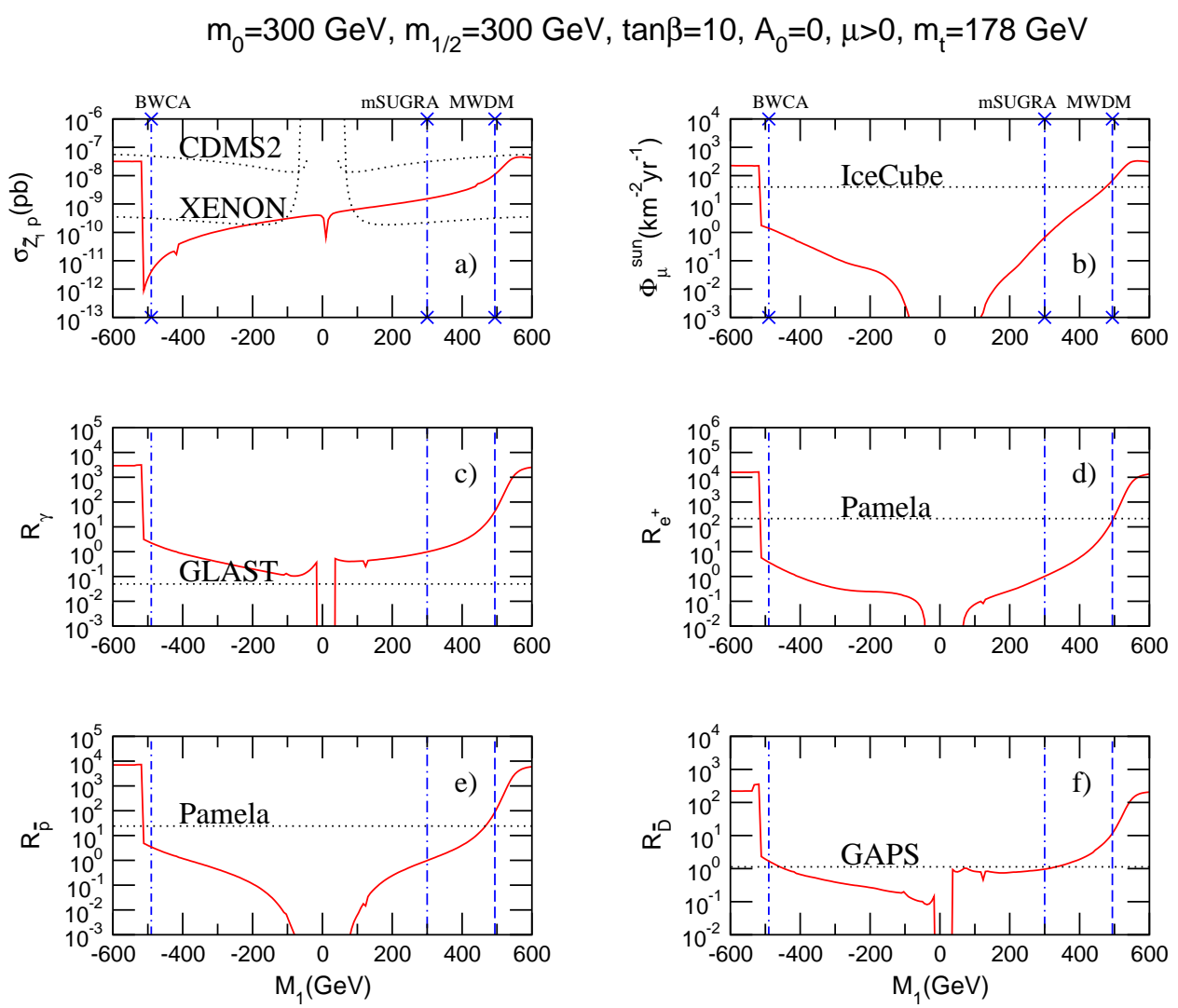

Figure 10: Rates for direct and indirect detection of neutralino dark matter vs. $M_{1}$ for $m_{0}=$ $m_{1 / 2}=300 \mathrm{GeV}$, with $\tan \beta=10, A_{0}=0, \mu>0$. Frames $\left.c\right)-f$ ) show the ratio of indirect detection rates compared to the mSUGRA model. In this plot, we adopt the adiabatically contracted N03 distribution for halo dark matter.

scattering cross section plummets to values in the $10^{-12} \mathrm{pb}$ range, far below the sensitivity of any planned detector. The drop-off is due to increasing negative interference amongst the contributing Feynman diagrams.

In frame $b$ ), we show the flux of muons from neutralino pair annihilations in the core of the Sun. The muon flux is below the reach of IceCube in the mSUGRA case, and it remains below IceCube observability in the BWCA case. The rate for neutralino annihilation in the sun or earth is given by

$$
\Gamma_{A}=\frac{1}{2} C \tanh ^{2}\left(\frac{t_{\odot}}{\tau}\right)
$$

where $C$ is the capture rate, $A$ is the total annihilation rate times relative velocity per volume, $t_{\odot}$ is the present age of the solar system and $\tau=1 / \sqrt{C A}$ is the equilibration time. For small $C$, as shown in frame $a$ ), the equilibration time becomes large, so that $\Gamma_{A} \sim \frac{1}{2} C^{2} A t^{2}$, and is hence sensitive to the neutralino annihilation cross section times relative velocity, unlike cases where the neutralino-nucleon scattering cross section is large. The muon flux jumps to observable levels at more negative values of $M_{1}$, but this is only because the $\widetilde{Z}_{1}$ suddenly becomes wino-like, so that the relic density becomes too low. 
In frames $c), d), e$ ) and $f$ ) we show the flux of photons, positrons, antiprotons and antideuterons, respectively. The results here are plotted as ratios of fluxes normalized to the mSUGRA point, in order to give results that are approximately halo-model independent. (We do show the above described expected experimental reach lines as obtained by using the Adiabatically Contracted N03 Halo model [50].) The rates for indirect detection via observation of halo annihilation remnants are typically low in the BWCA scenario, since the bino annihilation cross sections are $s$-wave suppressed. Observable results are indicated for $\gamma$ rays by GLAST, but this is due in part to the very favorable N03 halo distribution which is assumed.

In figure 11, we show the same direct and indirect DM detection rates as in figure 10, except this time versus $M_{2}$ instead of $M_{1}$. In frame $a$ ), the neutralino-nucleon scattering rates do not have negative interference, and can remain at observable, although not enhanced, levels. The rates for detection of BWCA DM at IceCube are relatively low, as are rates for anti-matter detection by Pamela in frames $d$ ) and $e$ ). The rates for $\gamma$ detection by GLAST in frame $c$ ) are similar to those from the mSUGRA case, while the rate for antideuteron detection by GAPS is just barely observable for BWCA DM in frame $f$ ).

Overall, prospects for direct or indirect detection of BWCA dark matter are generally at or below levels expected in the mSUGRA model. For this reason, we do not present direct and indirect detection rates in the $m_{0} v s . m_{1 / 2}$ plane. We also mention that the situation is sharply different in the MWDM scenarios where the corresponding rates are generally larger than in the mSUGRA model. Thus, a detection of a signal in the XENON or Pamela experiments could serve to discriminate between these scenarios, especially if we already have some information of the SUSY spectrum from collider experiments.

\section{Neutralino radiative decay in the BWCA scenario}

The loop-induced radiative decay width for $\widetilde{Z}_{2} \rightarrow \widetilde{Z}_{1} \gamma$ has been calculated in ref. 80, 81]. A thorough numerical analysis [82, 83] has shown that the radiative decay can be large and even dominant in certain regions of MSSM parameter space. A necessary (but not sufficient) condition for this is that all tree level two body decay modes of $\widetilde{Z}_{2}$ be kinematically forbidden. In this case, $\widetilde{Z}_{2}$ usually decays via $\widetilde{Z}_{2} \rightarrow \widetilde{Z}_{2} f \bar{f}$, where $f$ is a light SM fermion. However, if $\widetilde{Z}_{1}$ and $\widetilde{Z}_{2}$ are close in mass, the formally higher order two body radiative decay becomes competitive with the three body decays $\widetilde{Z}_{2} \rightarrow f \bar{f} \widetilde{Z}_{1}$. This is because $\Gamma\left(\widetilde{Z}_{2} \rightarrow \widetilde{Z}_{1} \gamma\right) \propto\left(1-m_{\widetilde{Z}_{1}} / m_{\widetilde{Z}_{2}}\right)^{3}$, while $\Gamma\left(\widetilde{Z}_{2} \rightarrow \widetilde{Z}_{1} f \bar{f}\right) \propto\left(1-m_{\widetilde{Z}_{1}} / m_{\widetilde{Z}_{2}}\right)^{5}$.

As we have seen, in both the MWDM and BWCA scenarios $m_{\widetilde{Z}_{2}}-m_{\widetilde{Z}_{1}}$ is small, so that we may expect that the branching fraction for radiative decays may be enhanced. Moreover, in both cases, the couplings of the neutralinos to the $Z$ boson, which occur only via the higgsino components of the neutralino are strongly suppressed so that virtual $Z$ boson exchange contribution to three body decay amplitudes is correspondingly suppressed. However, vector boson-gaugino loops essentially also decouple from the radiative decay in the BWCA case because the bino does not couple to these. These do not, however, decouple in the MWDM case since both the photino and the zino couple to $W^{ \pm} \widetilde{W}^{\mp}$ system. The 

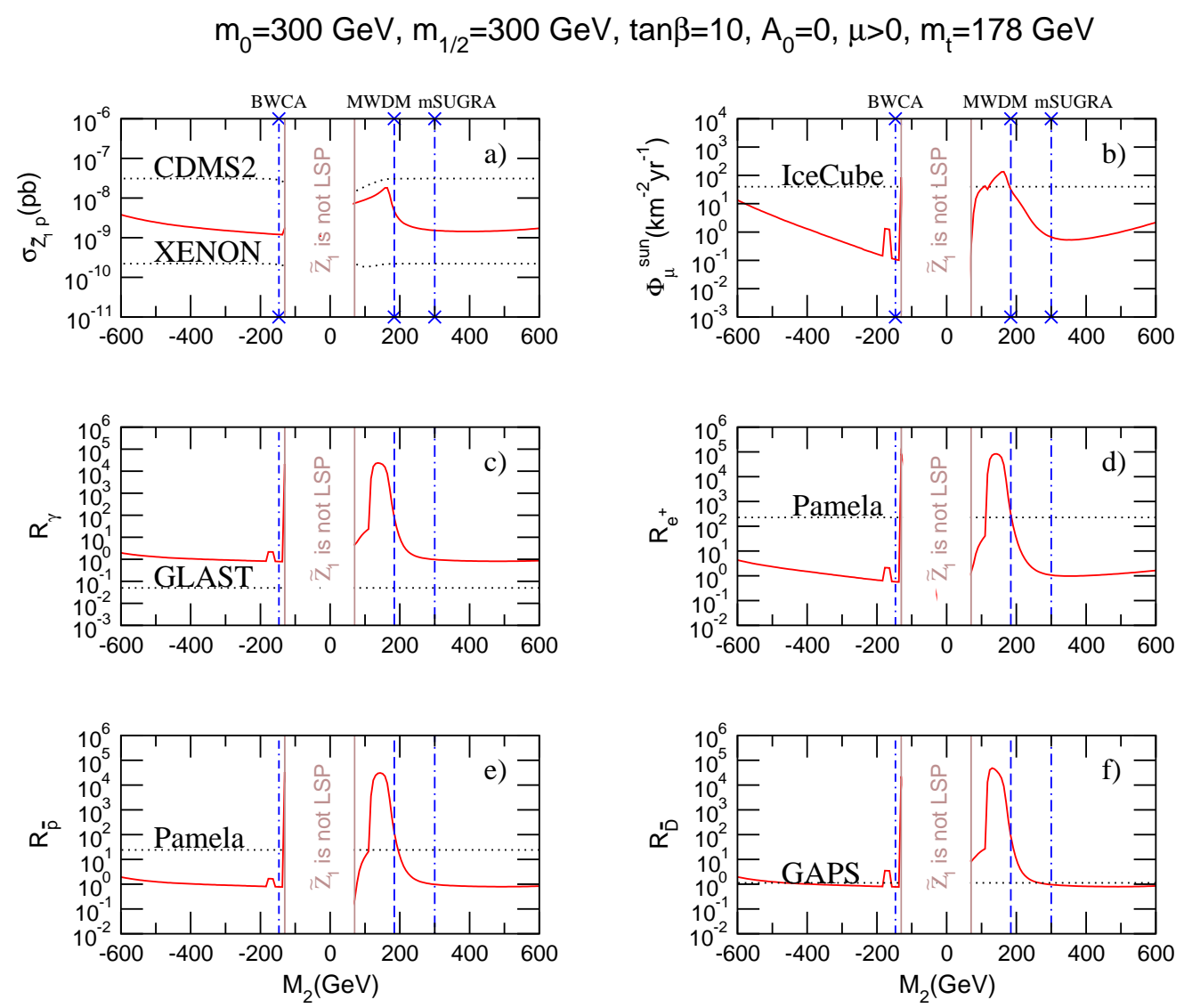

Figure 11: Rates for direct and indirect detection of neutralino dark matter vs. $M_{2}$ for $m_{0}=$ $m_{1 / 2}=300 \mathrm{GeV}$, with $\tan \beta=10, A_{0}=0, \mu>0$. Frames $\left.c\right)-f$ ) show the ratio of indirect detection rates compared to the mSUGRA model. In this plot, we adopt the adiabatically contracted N03 distribution for halo dark matter.

branching fraction for the radiative decay is thus a result of a complicated interplay between the kinematic and dynamic effects discussed above.

In figure 12, we show in upper frame $a$ ) the neutralino relic density, and in $b$ ) the $B F\left(\widetilde{Z}_{2} \rightarrow \widetilde{Z}_{1} \gamma\right)$ for the same parameters as in figure 1, versus GUT scale gaugino mass $M_{1}$. The radiative branching fraction at this point in the mSUGRA model is just $\sim 10^{-5}$. As $M_{1}$ climbs to $490 \mathrm{GeV}$, in the MWDM case, the branching fraction has climbed to $\sim 5 \%$. When $M_{1}$ varies to large negative values in the BWCA case, the branching fraction has climbed to $10 \%$. In this case, we may expect that a considerable fraction of SUSY events at colliders to contain hard isolated photons via the decay of $\widetilde{Z}_{2}$ that is produced either directly, or via cascade decays of heavier sparticles. In the two lower frames, we show the same figures except for large $m_{0}=1 \mathrm{TeV}$. In this case, the sfermion loops mediating the $\widetilde{Z}_{2} \rightarrow \widetilde{Z}_{1} \gamma$ decay become suppressed, and the branching fraction is much smaller, reaching $\sim 8 \%$ in the MWDM case, and just $1 \%$ in the BWCA case.

A similar situation is illustrated in figure 13, where now we plot versus variable $M_{2}$, while keeping $M_{1}=m_{1 / 2}=300 \mathrm{GeV}$. In the upper frames for $m_{0}=300 \mathrm{GeV}$, we see that while $B F\left(\widetilde{Z}_{2} \rightarrow \widetilde{Z}_{1} \gamma\right)$ reaches $5 \%$ in the MWDM case, it reaches $25 \%$ in the BWCA 

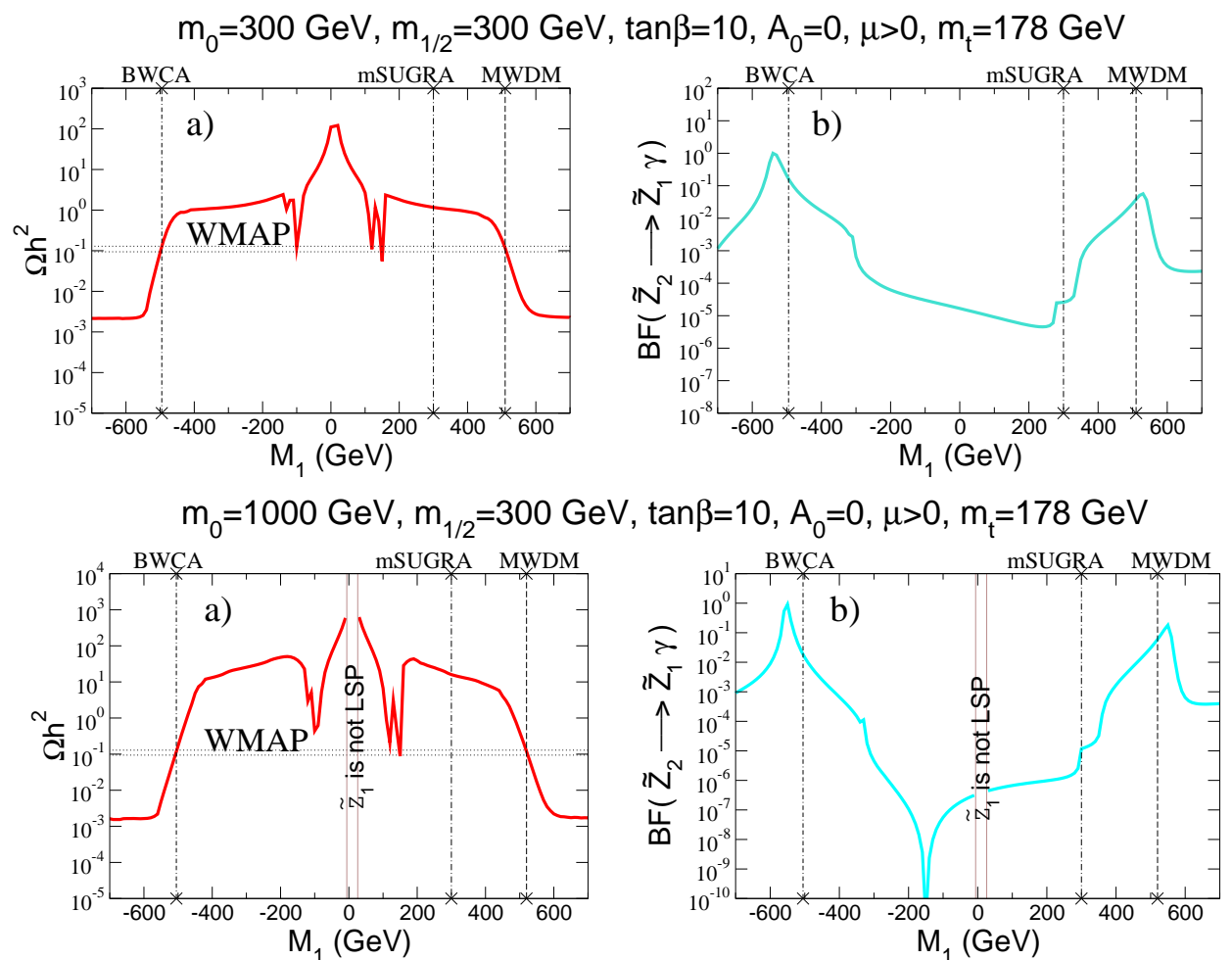

Figure 12: Upper: A plot of $a)$ relic density $\Omega_{C D M} h^{2}$ and $\left.b\right) B F\left(\widetilde{Z}_{2} \rightarrow \widetilde{Z}_{1} \gamma\right)$ as a function of $M_{1}$ for $m_{0}=300 \mathrm{GeV}, m_{1 / 2}=300 \mathrm{GeV}, A_{0}=0, \tan \beta=10, \mu>0$ and $m_{t}=178 \mathrm{GeV}$. Lower: Same plot for $m_{0}=1000 \mathrm{GeV}$.

scenario. In the lower frames, we see that for $m_{0}=1 \mathrm{TeV}$, the branching fraction reaches $8 \%$ for the case of MWDM and $0.8 \%$ for BWCA dark matter. We note that in both figures $B\left(\widetilde{Z}_{2} \rightarrow \widetilde{Z}_{1} \gamma\right)$ attains a higher value at its peak when $M_{1} / M_{2}$ is negative because it is in this case that the sparticle masses get really close (see the level crossings in figures 0 and 5 ).

In figure 14, we plot contours of $B F\left(\widetilde{Z}_{2} \rightarrow \widetilde{Z}_{1} \gamma\right)$ in the $m_{0}$ vs. $m_{1 / 2}$ plane for $A_{0}=0$, $\tan \beta=10, \mu>0$ and $m_{t}=178 \mathrm{GeV}$. At every point in the plane of frame $a$ ), we have adjusted $M_{1}$ to a negative value chosen so that $\Omega_{\widetilde{Z}_{1}} h^{2}=0.11$ in the BWCA scenario. We see that the $B F\left(\widetilde{Z}_{2} \rightarrow \widetilde{Z}_{1} \gamma\right)$ exceeds $50 \%$ around $m_{0} \sim 600-1000 \mathrm{GeV}$ for $m_{1 / 2} \sim$ $1-1.4 \mathrm{TeV}$. The branching fraction remains large at all $m_{1 / 2}$ values, but diminishes for $m_{0} \gtrsim 500-1000 \mathrm{GeV}$. In figure $14 b$ ), we plot the same contours except that at every point in the plane, we have instead adjusted $M_{2}$ to negative values so that $\Omega_{\widetilde{Z}_{1}} h^{2}=0.11$. In this case, we see again that the branching fraction can become larger than 0.3 for low $m_{0}$ values. In frames $c$ ) and $d$ ) once again we show contours of $B\left(\widetilde{Z}_{2} \rightarrow \widetilde{Z}_{1} \gamma\right)$ but for the case of MWDM, where $M_{1}$ and $M_{2}$ are dialed to positive values. While the radiative branching fractions never reach much beyond the $10 \%$ level for MWDM, they maintain a significant rate out to large values of $m_{0}$. This is because in the MWDM case the radiative loop decays are dominated by $W$-chargino exchange, whereas in the BWCA case the radiative loops are dominated by fermion-sfermion exchange. 

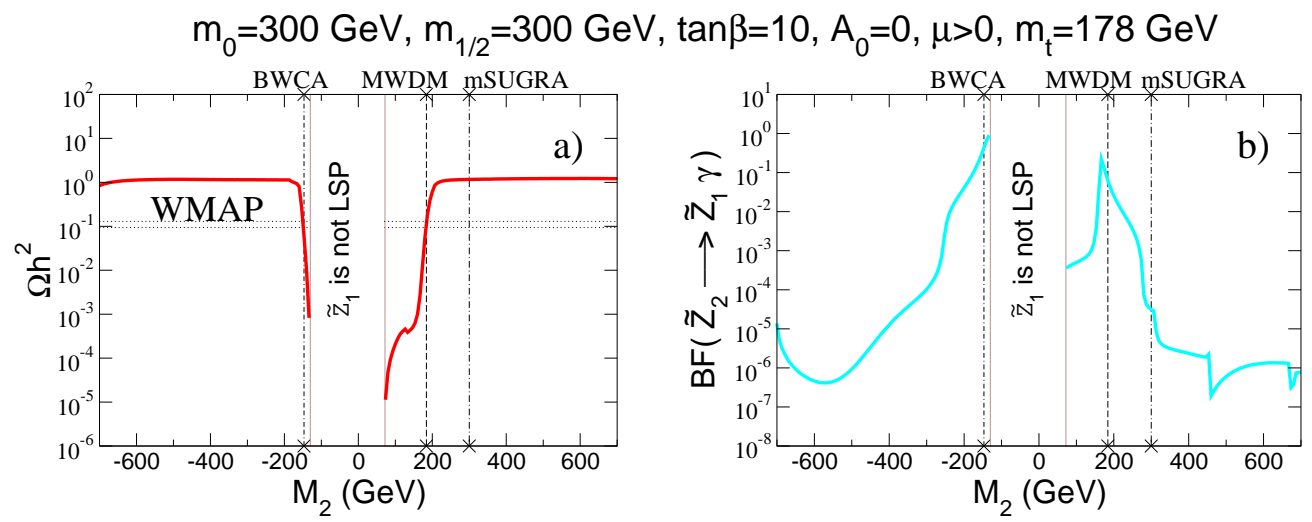

$m_{0}=1000 \mathrm{GeV}, m_{1 / 2}=300 \mathrm{GeV}, \tan \beta=10, A_{0}=0, \mu>0, m_{t}=178 \mathrm{GeV}$
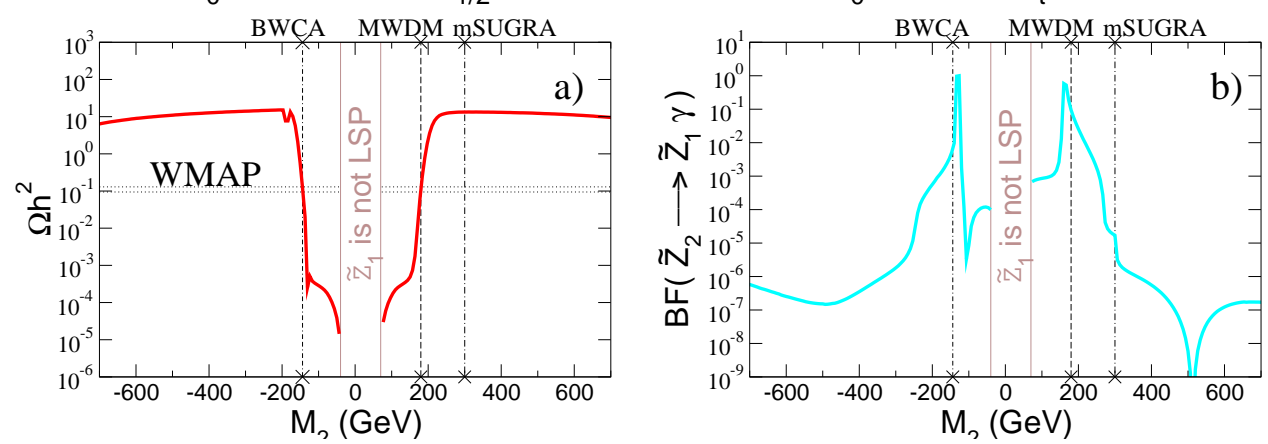

Figure 13: Upper: A plot of $a)$ relic density $\Omega_{C D M} h^{2}$ and $\left.b\right) B F\left(\widetilde{Z}_{2} \rightarrow \widetilde{Z}_{1} \gamma\right)$ as a function of $M_{2}$ for $m_{0}=300 \mathrm{GeV}, m_{1 / 2}=300 \mathrm{GeV}, A_{0}=0, \tan \beta=10, \mu>0$ and $m_{t}=178 \mathrm{GeV}$. Lower: Same plot for $m_{0}=1000 \mathrm{GeV}$.

\section{BWCA dark matter at colliders}

\section{$5.1 \widetilde{Z}_{2}-\widetilde{Z}_{1}$ mass gap in BWCA scenario}

An important question is whether collider experiments would be able to distinguish the case of BWCA dark matter from other forms of neutralino DM such as MHDM as occur in the mSUGRA model or MWDM. We have seen from the plots of sparticle mass spectra that the squark and gluino masses vary only slightly with changing $M_{1}$ or $M_{2}$. However, the chargino and neutralino masses change considerably, and in fact rather small mass gaps $m_{\widetilde{W}_{1}}-m_{\widetilde{Z}_{1}}$ and $m_{\widetilde{Z}_{2}}-m_{\widetilde{Z}_{1}}$ are in general expected in both BWCA and MWDM scenarios, as compared to the case of models that incorporate gaugino mass unification close to the GUT scale.

In figure 15, we show contours of the mass gap $m_{\widetilde{Z}_{2}}-m_{\widetilde{Z}_{1}}$ in the $m_{0}$ vs. $m_{1 / 2}$ plane for $A_{0}=0, \tan \beta=10$ and $\mu>0$ for $a$ ) the mSUGRA model, $b$ ) the case of BWCA DM where $-M_{1}$ is raised at every point until $\Omega_{\widetilde{Z}_{1}} h^{2} \rightarrow 0.11$ and $c$ ) the case of BWCA DM where $-M_{2}$ is lowered until $\Omega_{\widetilde{Z}_{1}} h^{2} \rightarrow 0.11$. In the case of the mSUGRA model, most of the parameter space has $m_{\widetilde{Z}_{2}}-m_{\widetilde{Z}_{1}}>90 \mathrm{GeV}$, which means that $\widetilde{Z}_{2} \rightarrow \widetilde{Z}_{1} Z^{0}$ decay is allowed. When this decay is allowed, its branching fraction is always large, unless it competes with other two-body decays such as $\widetilde{Z}_{2} \rightarrow \widetilde{Z}_{1} h$ or $\widetilde{Z}_{2} \rightarrow \bar{f} \tilde{f}$ or $f \tilde{\tilde{f}}$ (where $f$ is 

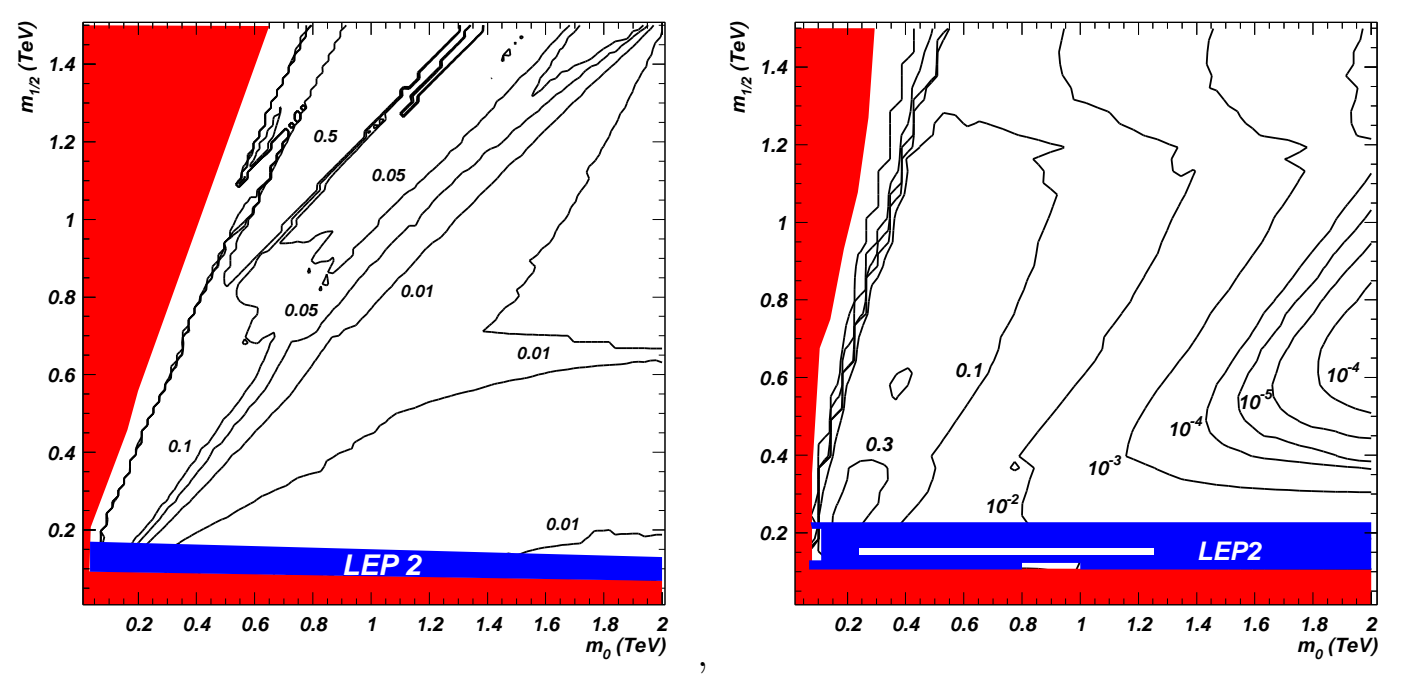

MWDM: $M_{1} \neq m_{1 / 2}, \tan \beta=10, A_{0}=0, \mu>0, m_{t}=178 \mathrm{GeV}$

MWDM: $M_{2} \neq m_{1 / 2}, \tan \beta=10, A_{0}=0, \mu>0, m_{t}=178 \mathrm{GeV}$
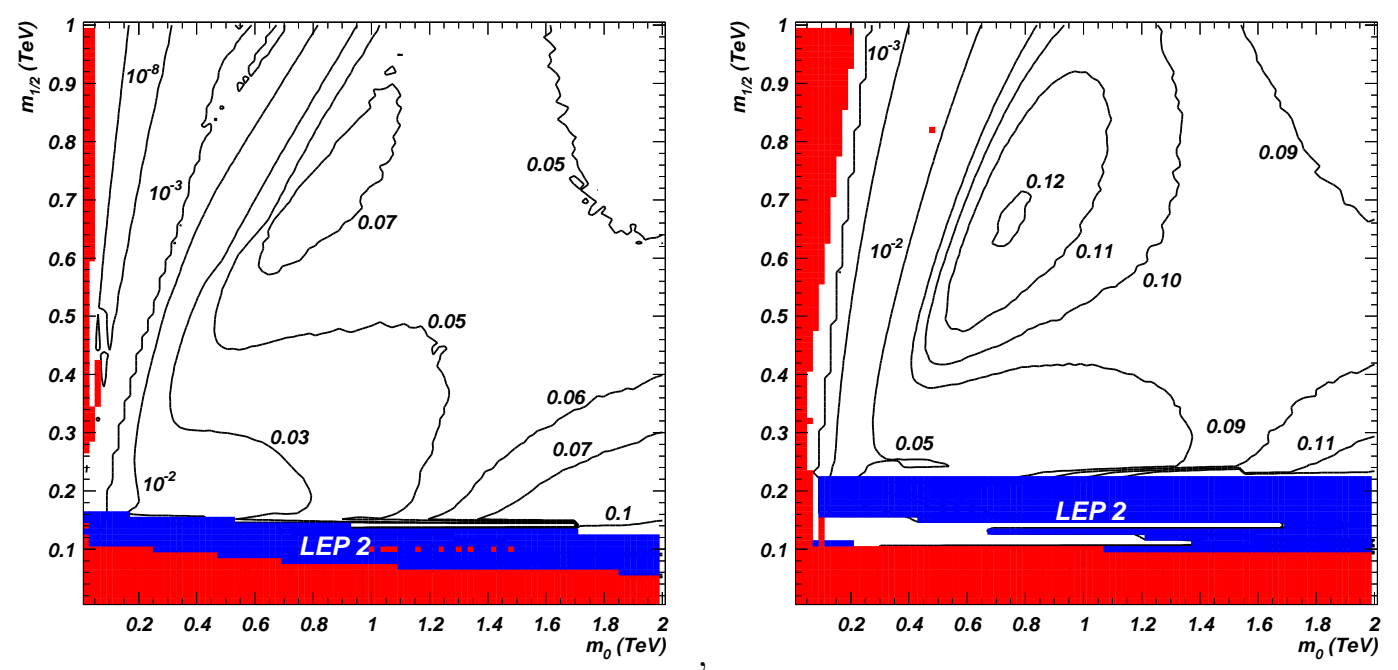

Figure 14: Contours of $B F\left(\widetilde{Z}_{2} \rightarrow \widetilde{Z}_{1} \gamma\right)$ in the $m_{0}$ vs. $m_{1 / 2}$ plane for $\tan \beta=10, A_{0}=0, \mu>0$, $m_{t}=178 \mathrm{GeV}$. In $a$ )., we have adjusted $M_{1}$ everywhere to negative values so that $\Omega_{\widetilde{Z}_{1}} h^{2}=0.11$ in the BWCA scenario. In $b$ )., we have adjusted $M_{2}$ everywhere to negative values so that $\Omega_{\widetilde{Z}_{1}} h^{2}=$ 0.11 in the BWCA scenario. In $c)$. $(d)$.), we have adjusted $M_{1}$ and $\left(M_{2}\right)$ everywhere to positive values so that $\Omega_{\widetilde{Z}_{1}} h^{2}=0.11$ in the MWDM scenario.

a SM fermion). In the case of BWCA DM in frames $b$ ) and $c$ ), we see that (aside from the left-most portion of frame $b$ ), which is not a region of BWCA), the mass gap is much smaller, so that two-body tree level decays of $\widetilde{Z}_{2}$ and $\widetilde{W}_{1}$ are closed and three-body decays are dominant. If $\widetilde{Z}_{2}$ 's are produced at large rates either directly or via gluino or squark cascade decays [84], it should be possible to identify opposite sign/ same flavor dilepton pairs from their decays, to reconstruct their invariant mass, and extract the upper edge of the invariant mass distribution 85, 86.

Finally, we note one curious feature of figure $15 c$ that was referred to in the caption of 

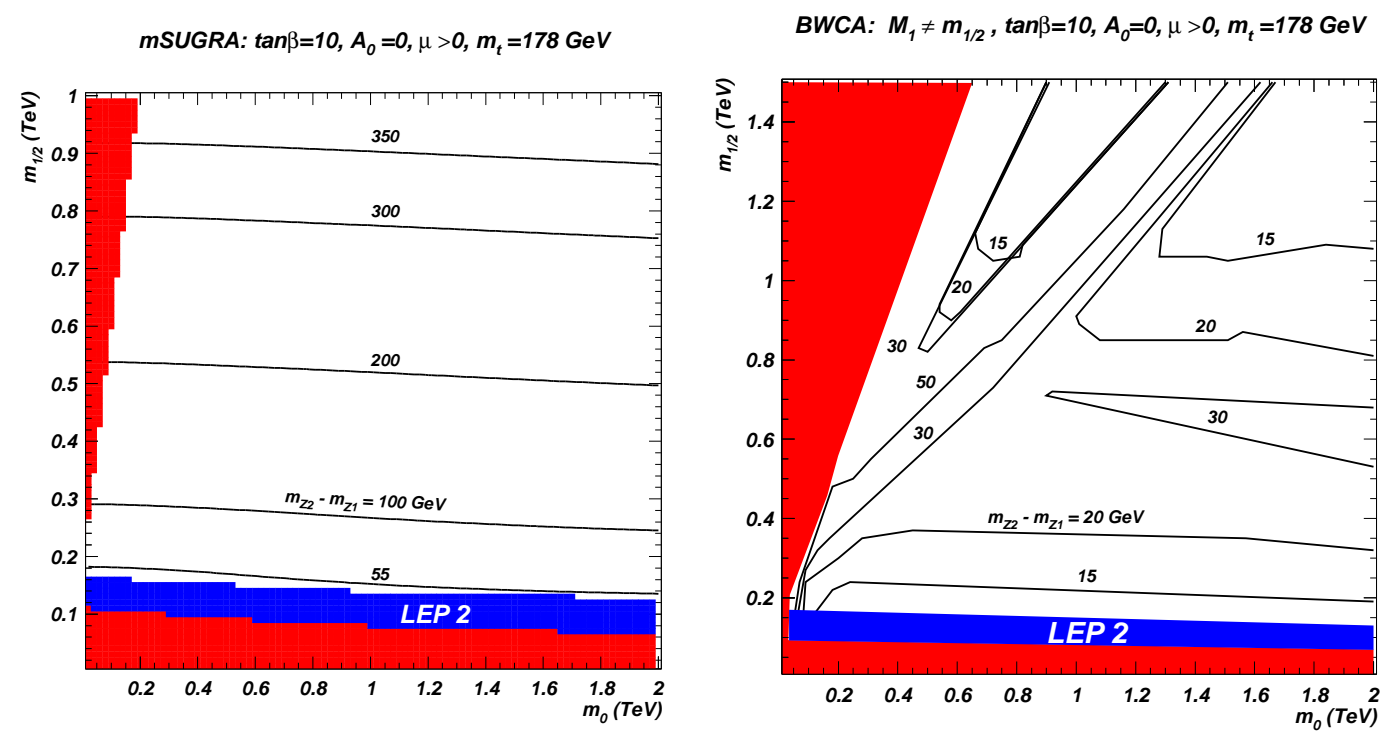

BWCA: $M_{2} \neq m_{1 / 2}, \tan \beta=10, A_{0}=0, \mu>0, m_{t}=178 \mathrm{GeV}$

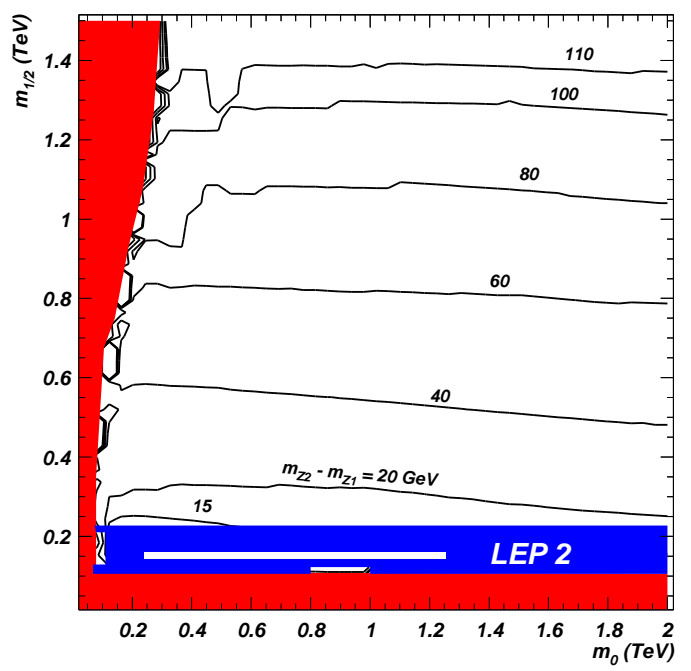

Figure 15: Contours of $m_{\widetilde{Z}_{2}}-m_{\widetilde{Z}_{1}}$ mass gap in the $m_{0}$ vs. $m_{1 / 2}$ plane for $\tan \beta=10, A_{0}=0$, $\mu>0$ and $a$ ) mSUGRA model, $b)-M_{1}>m_{1 / 2}$ BWCA and $\left.c\right)-M_{2}<m_{1 / 2}$ BWCA.

figure 6. Within the blue shaded LEP2 excluded region there appear three allowed strips. The lower horizontal strip at $m_{1 / 2} \sim 100 \mathrm{GeV}$ corresponds to the neutralino $Z$-annihilation funnel, where $M_{2}$ does not have to be dialed to low values, since $Z$ annihilation already reduces the relic density. However, in this case where $M_{2}$ is negative, terms in the two-loop gaugino mass RGE conspire to yield a weak scale $\left|M_{2}\right|$ value which is somewhat larger than its mSUGRA counterpart. This means that while $2 m_{\widetilde{Z}_{1}} \sim M_{Z}$, at the same time $m_{\widetilde{W}_{1}}$ is just above $103.5 \mathrm{GeV}$, and is thus LEP2 allowed. A similar situation occurs for the longer allowed strip at $m_{1 / 2} \sim 150 \mathrm{GeV}$, except in this case it is the $h$ resonance which reduces the relic density. Finally, at very low $m_{0} \sim 80 \mathrm{GeV}$, bulk annihilation via light sleptons reduces the relic density, while the negative $M_{2}$ pushed $m_{\widetilde{W}_{1}}$ above the LEP2 limit. The corresponding strips do not appear in frame $b$ ) because here, we have only scanned $M_{1}$ 
values above $m_{1 / 2}$, as required to get agreement with WMAP: as we can see from figure [ that the $Z$ and $h$ resonances appear for $\left|M_{1}\right|<m_{1 / 2}$.

\subsection{Fermilab Tevatron}

In the mSUGRA model, the best reach for SUSY at the Fermilab Tevatron occurs in the clean trilepton channel [87, 88]. We examined the clean trilepton signal rate for case study point BWCA3. For this point, the total SUSY particle production cross section was $\sim 440 \mathrm{fb}$ for $\sqrt{s}=2 \mathrm{TeV} p \bar{p}$ collisions. Using the soft trilepton cuts $\mathrm{SC} 2$ of ref. 88. (three isolated leptons with $p_{T}\left(\ell_{1}, \ell_{2}, \ell_{3}\right)>11,7,5 \mathrm{GeV},\left|\eta\left(\ell_{1,2 / 3}\right)\right|<1,2, E_{T}^{\text {miss }}>25 \mathrm{GeV}$, $m(\ell \bar{\ell})>20 \mathrm{GeV}, m_{T}\left(\ell, E_{T}^{\text {miss }}\right)<60 \mathrm{GeV}$ plus a $Z$ mass veto), we find a surviving signal cross section of only $0.035 \mathrm{fb}$, well below observability. The main problem here is the $m(\ell \bar{\ell})>20 \mathrm{GeV}$ cut. This cut is essential to remove background from virtual photons in $q \bar{q}^{\prime} \rightarrow \ell \nu_{\ell} \ell^{\prime} \bar{\ell}^{\prime}$ electroweak production. However, the cut also kills much of the SUSY signal in the BWCA case, since the $\ell \bar{\ell}$ invariant mass is constrained to be $<m_{\widetilde{Z}_{2}}-m_{\widetilde{Z}_{1}}$, which is already quite small.

Another possibility is to search for events at the Tevatron containing isolated high $E_{T}$ photons from radiative $\widetilde{Z}_{2}$ decays. We examined the isolated photon inclusive channel $\left(E_{T}(\gamma)>8 \mathrm{GeV}\right.$ with $\sum_{\text {cone }}^{R=0.4} E_{T}<0.1 E_{T}(\gamma)$ and $\left.|\eta(\gamma)|<1.1\right)$, the photon plus lepton channel $\left(p_{T}(\ell)>15 \mathrm{GeV}\right.$ with $\sum_{c o n e}^{R=0.4} E_{T}<5 \mathrm{GeV}$ and $\left.E_{T}^{\text {miss }}>20 \mathrm{GeV}\right)$, and photon plus $E_{T}^{\text {miss }}>20 \mathrm{GeV}$ channels for the BWCA3 case study (see table 2). In the latter two channels, we also vetoed jets. The signal rates were $43,2.7$ and $13.5 \mathrm{fb}$ respectively, while backgrounds from $W \gamma(Z \gamma)$ production were 6911 (1803), 2500 (6.2) and 394 (253) fb, respectively. In light of these results, it appears very difficult to use the isolated photon channels in the BWCA scenario to identify SUSY at the Tevatron.

\subsection{CERN LHC}

If the $R$-parity conserving MSSM is a good description of nature at the weak scale, then multi-jet plus multi-lepton plus $E_{T}^{\text {miss }}$ events should occur at large rates at the CERN LHC, provided that $m_{\tilde{g}} \lesssim 2-3 \mathrm{TeV}$ [89]. The LHC reach for SUSY in the mSUGRA model has been calculated in ref. 90, 91]. The ultimate mSUGRA reach results, coming from the $E_{T}^{\text {miss }}+$ jets channel, should also apply qualitatively to the BWCA case, since the values of $m_{\tilde{g}}$ and $m_{\tilde{q}}$ change little in going from mSUGRA to BWCA, and the $E_{T}^{\text {miss }}+$ jets reach mainly depend on these masses.

The reach in other channels such as multileptons plus jets and isolated photons plus jets may change substantially in the BWCA scenario. The reach of the LHC in the $\left(m_{0}, m_{1 / 2}\right)$ plane of the mSUGRA model was recently re-assessed in ref. 91]. The search strategy was based on the detection of gluino and squark cascade decay products, namely multiple high transverse momentum jets and/or leptons and/or photons plus large missing transverse energy. Here, we use Isajet $7.72^{\prime}$ [39] for the simulation of signal and background events at the LHC. The event and detector simulation was performed along the lines established in ref. [91], where details on cuts and detector resolution along with our definitions of jets and isolated leptons and photons may be found. 
$B W C A: M_{2} \neq m_{1 / 2}, \tan \beta=10, A_{0}=0, \mu>0, m_{t}=178 \mathrm{GeV}$

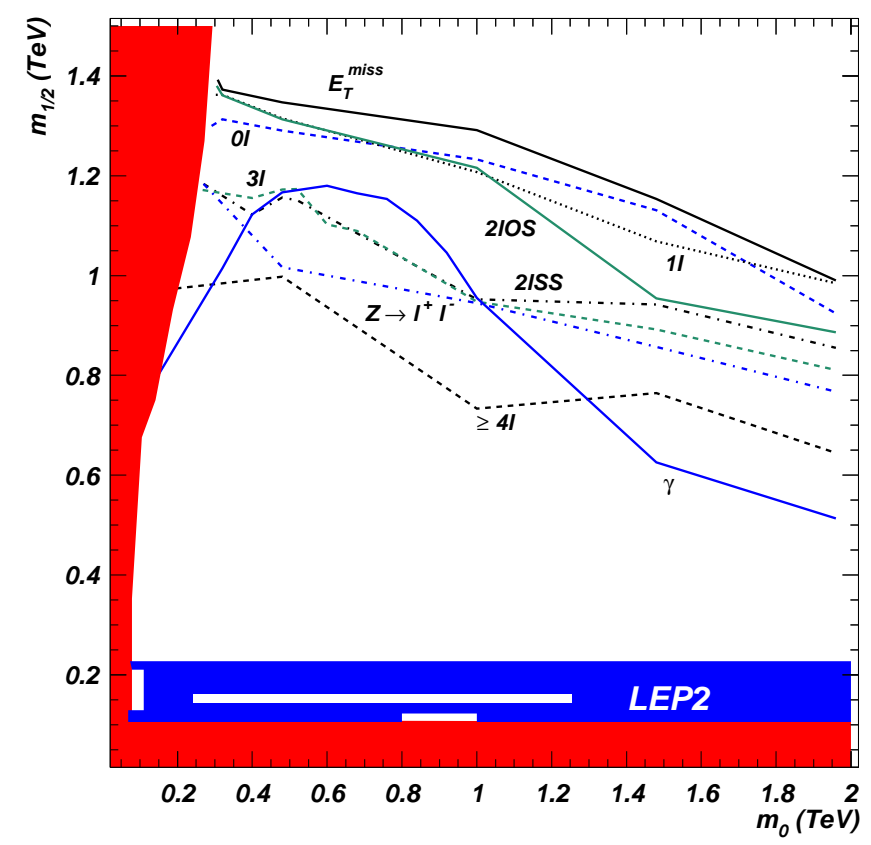

Figure 16: Reach contours of the CERN LHC for $100 \mathrm{fb}^{-1}$ of integrated luminosity for various signal topologies in the $m_{0}$ vs. $m_{1 / 2}$ plane for $\tan \beta=10, A_{0}=0, \mu>0$ and for $-M_{2}<m_{1 / 2}$ such that $\Omega_{\widetilde{Z}_{1}} h^{2}=0.11$ at every point in the plane.

We plot the reach of the LHC in figure 16 for the BWCA case using the procedure described in [91]. All events had to pass the pre-cuts, which impose the requirement that $E_{T}^{\text {miss }}>200 \mathrm{GeV}$ and there are at least 2 jets with $p_{T}^{\text {jet }}>40 \mathrm{GeV}$. We then optimized the cuts using the strategy in ref. [91] - generally speaking, harder jet $E_{T}$ and $E_{T}^{\text {miss }}$ cuts apply for heavier sparticles, and softer cuts apply for lighter sparticles. The events are divided into several classes, characterized by the number of leptons or the presence of an isolated photon in the final state. The $5 \sigma$ discovery reach for $100 \mathrm{fb}^{-1}$ of integrated luminosity is shown for the various channels. The ultimate reach in the $E_{T}^{\text {miss }}+$ jets channel ranges from $m_{1 / 2} \sim 1.4 \mathrm{TeV}$ at low $m_{0}$ to $m_{1 / 2} \sim 1 \mathrm{TeV}$ at $m_{0}=2 \mathrm{TeV}$. These results are similar to those obtained in the mSUGRA model. The reach contour in the isolated photon plus jets plus $E_{T}^{\text {miss }}$ channel, however, has greatly increased in the BWCA scenario compared to the mSUGRA case. In mSUGRA, the reach contour varies from $m_{1 / 2}: 850 \rightarrow 500 \mathrm{GeV}$ as $m_{0}: .1 \rightarrow 2 \mathrm{TeV}$. In the BWCA case, where $\widetilde{Z}_{2} \rightarrow \widetilde{Z}_{1} \gamma$ at a large rate, the photon reach contour reaches a maximum of $m_{1 / 2}=1.2 \mathrm{TeV}$ for $m_{0} \sim 500 \mathrm{GeV}$. Thus, at CERN LHC, the BWCA scenario will be signalled by multijet plus isolated multilepton plus $E_{T}^{\text {miss }}$ events, but with a large content of hard isolated photons as well, at least for the case where $m_{0} \lesssim 1 \mathrm{TeV}{ }^{8}$ For larger values of $m_{0}>1 \mathrm{TeV}$, the $\widetilde{Z}_{2} \rightarrow \widetilde{Z}_{1} \gamma$ branching fraction

\footnotetext{
${ }^{8}$ Gauge mediated SUSY breaking (GMSB) models in which the next-to-lightest SUSY particle (NLSP) decays to a photon and a gravitino also have photons in SUSY events. It is unlikely that these models will be confused with the BWCA scenario because not only is the sparticle mass spectrum quite different
} 
drops, and the reach projections become similar to the case of mSUGRA. We have also checked that the reach using the $\gamma+n$ lepton channel is smaller than the reach via the corresponding $n$ lepton channel, primarily because the signal becomes too small to pass our 10 event $/ 100 \mathrm{fb}^{-1}$ requirement.

For SUSY searches at the CERN LHC, Hinchliffe et al. have pointed out 866 that an approximate value of $m_{\tilde{q}}$ or $m_{\tilde{g}}$ can be gained by extracting the maximum in the $M_{\text {eff }}$ distribution, where $M_{\text {eff }}=E_{T}^{\text {miss }}+E_{T}($ jet 1$)+E_{T}($ jet 2$)+E_{T}($ jet 3$)+E_{T}($ jet 4$)$. Their analysis will carry over to the BWCA scenario, as well as in models with gaugino mass unification, so that the approximate mass scale of strongly interacting sparticles will be known soon after a supersymmetry signal has been established.

In mSUGRA, a dilepton mass edge should be visible in SUSY signal events only if $m_{1 / 2} \lesssim 250 \mathrm{GeV}$ or if $\widetilde{Z}_{2} \rightarrow \tilde{\ell} \bar{\ell}, \overline{\tilde{\ell} \ell}$ decays are allowed. In the case of BWCA DM, as with MWDM, the dilepton mass edge should be visible over almost all parameter space. We illustrate the situation for four case studies listed in table $2{ }^{9}$ The first case, labeled mSUGRA, has $m_{0}=m_{1 / 2}=300 \mathrm{GeV}$, with $A_{0}=0, \tan \beta=10$ and $\mu>0$. In this case, $\tilde{g} \tilde{g}$, $\tilde{g} \tilde{q}$ and $\tilde{q} \tilde{q}$ production occurs with a combined cross section of about $12 \mathrm{pb}$, while the total SUSY cross section is around $13.4 \mathrm{pb}$ (the additional $1.4 \mathrm{pb}$ comes mainly from -ino pair production and -ino-squark or -ino-gluino associated production). The case of BWCA1, with $M_{1}=-480 \mathrm{GeV}$, has similar rates of sparticle pair production. The case of BWCA2, with lighter chargino and neutralino masses, has a total production cross section of $19.2 \mathrm{pb}$, wherein strongly interacting sparticles are pair produced at similar rates as in mSUGRA or BWCA1, but -ino pairs are produced at a much larger rate $\sim 6.1 \mathrm{pb}$. We also show the case of BWCA3, which is similar to that of BWCA2 except that $\mu<0$, which gives a better fit to $(g-2)_{\mu}$ measurements.

We have generated 50K LHC SUSY events for each of these cases using Isajet $7.72^{\prime}$, and passed them through a toy detector simulation as described above. Since gluino and squark masses of the three case studies are similar to those of LHC point 5 of the study of Hinchliffe et al.,frank, we adopt the same overall signal selection cuts which efficiently select the SUSY signal while essentially eliminating SM backgrounds: $E_{T}^{\text {miss }}>\max \left(100 \mathrm{GeV}, 0.2 M_{\text {eff }}\right)$, at least four jets with $E_{T}>50 \mathrm{GeV}$, where the hardest jet has $E_{T}>100 \mathrm{GeV}$, transverse sphericity $S_{T}>0.2$ and $M_{\text {eff }}>800 \mathrm{GeV}$.

In these events, we require at least two isolated leptons, and then plot the invariant mass of all same flavor/opposite sign dileptons. The results are shown in figure 17. In the case of the mSUGRA model, frame $a$ ), there is a sharp peak at $m\left(\ell^{+} \ell^{-}\right) \sim M_{Z}$,

but the photons in the GMSB scenario would typically have much larger energy because the gravitino is essentially massless. Moreover, unless the NLSP has many decay modes, the multiplicity of photons in the GMSB case would be much larger since every SUSY event would contain two photons.

${ }^{9}$ In this study, a toy detector simulation is employed with calorimeter cell size $\Delta \eta \times \Delta \phi=0.05 \times 0.05$ and $-5<\eta<5$. The hadronic energy resolution is taken to be $80 \% / \sqrt{E}$ for $|\eta|<2.6$ and $100 \% / \sqrt{E}$ for $|\eta|>2$.6. The electromagnetic energy resolution is assumed to be $3 \% / \sqrt{E}$. We use a UA1-like jet finding algorithm with jet cone size $R=0.5$ and $p_{T}^{\text {jet }}>25 \mathrm{GeV}$. We also require that $\left|\eta_{\ell}\right|<2.5$ and $\left|\eta_{j}\right|<3$. Leptons (es or $\mu \mathrm{s}$ ) have to also satisfy $p_{T}^{\text {lepton }} \geq 10 \mathrm{GeV}$. Leptons are considered isolated if the visible activity within the cone $\Delta R<0.3$ is $\Sigma E_{T}^{\text {cells }}<2 \mathrm{GeV}$. The strict isolation criterion helps reduce multi-lepton background from heavy quark (especially $t \bar{t}$ ) production. 


\begin{tabular}{|lcccc|}
\hline parameter & mSUGRA & BWCA1 & BWCA2 & BWCA3 \\
\hline$M_{1}$ & 300 & -480 & 300 & 300 \\
$M_{2}$ & 300 & 300 & -156 & -170 \\
$\mu$ & 409.2 & 401.3 & 402.3 & -401.6 \\
$m_{\tilde{g}}$ & 732.1 & 733.4 & 736.3 & 736.8 \\
$m_{\tilde{u}_{L}}$ & 713.9 & 715.3 & 701.9 & 703.5 \\
$m_{\tilde{t}_{1}}$ & 523.4 & 535.0 & 554.8 & 566.4 \\
$m_{\tilde{b}_{1}}$ & 650.0 & 651.8 & 645.1 & 646.1 \\
$m_{\tilde{e}_{L}}$ & 364.7 & 371.6 & 324.5 & 327.7 \\
$m_{\tilde{e}_{R}}$ & 322.8 & 352.7 & 322.6 & 322.6 \\
$m_{\widetilde{W}_{2}}$ & 432.9 & 426.0 & 419.6 & 421.3 \\
$m_{\widetilde{W}_{1}}$ & 223.9 & 223.4 & 138.5 & 141.7 \\
$m_{\widetilde{Z}_{4}}$ & 433.7 & 425.0 & 415.2 & 419.7 \\
$m_{\widetilde{Z}_{3}}$ & 414.8 & 409.4 & 414.0 & 410.0 \\
$m_{\widetilde{Z}_{2}}$ & 223.7 & 222.7 & 138.6 & 141.4 \\
$m_{\widetilde{Z}_{1}}$ & 117.0 & 200.0 & 116.8 & 118.8 \\
$m_{A}$ & 538.7 & 537.1 & 508.4 & 508.5 \\
$m_{H^{+}}$ & 548.0 & 546.4 & 517.9 & 518.0 \\
$m_{h}$ & 115.7 & 115.3 & 114.0 & 112.7 \\
$\Omega_{\widetilde{Z}_{1}} h^{2}$ & 1.1 & 0.11 & 0.10 & 0.12 \\
$B F(b \rightarrow s \gamma)$ & $3.2 \times 10^{-4}$ & $3.3 \times 10^{-4}$ & $3.7 \times 10^{-4}$ & $4.6 \times 10^{-4}$ \\
$\Delta a_{\mu}$ & $12.1 \times 10^{-10}$ & $9.9 \times 10^{-10}$ & $-13.8 \times 10^{-10}$ & $13.1 \times 10^{-10}$ \\
$\sigma_{s c}\left(\widetilde{Z}_{1} p\right)$ & $1.5 \times 10^{-9} \mathrm{pb}$ & $4.7 \times 10^{-12} \mathrm{pb}$ & $1.2 \times 10^{-9} \mathrm{pb}$ & $8.0 \times 10^{-11} \mathrm{pb}$ \\
$B F\left(\widetilde{Z}_{2} \rightarrow \widetilde{Z}_{1} \gamma\right)$ & $2.7 \times 10^{-5}$ & 0.096 & 0.25 & 0.25 \\
\hline
\end{tabular}

Table 2: Masses and parameters in GeV units for mSUGRA and three BWCA scenarios. In each case, $m_{0}=m_{1 / 2}=300 \mathrm{GeV}, A_{0}=0, \tan \beta=10$ and $m_{t}=178 \mathrm{GeV}$. The third BWCA case has $\mu<0$, while the first two have $\mu>0$.

which comes from $\widetilde{Z}_{2} \rightarrow \widetilde{Z}_{1} Z^{0}$ decays where $\widetilde{Z}_{2}$ is produced in the gluino and squark cascade decays. In the case of BWCA1 in frame $b$ ), we again see a $Z^{0}$ peak, although here the $Z^{0}$ s arise from $\widetilde{Z}_{3}, \widetilde{Z}_{4}$ and $\widetilde{W}_{2}$ decays. We also see the continuum distribution in $m\left(\ell^{+} \ell^{-}\right)<m_{\widetilde{Z}_{2}}-m_{\widetilde{Z}_{1}}=22.7 \mathrm{GeV}$. The cross section plotted here is $\sim 0.035 \mathrm{pb}$, which would correspond to $3.5 \mathrm{~K}$ events in $100 \mathrm{fb}^{-1}$ of integrated luminosity (the sample shown in the figure contains just 135 events). In frame $c$ )- with a cross section of $\sim 0.07$ pb (but just 187 actual entries)- we see again the $Z^{0}$ peak, but also we see again the $m\left(\ell^{+} \ell^{-}\right)<m_{\widetilde{Z}_{2}}-m_{\widetilde{Z}_{1}}=21.8 \mathrm{GeV}$ continuum. In both these BWCA cases, the $m_{\widetilde{Z}_{2}}-m_{\widetilde{Z}_{1}}$ mass edge should be easily measurable. It should also be obvious that it is inconsistent with models based on gaugino mass unification, in that the projected ratios $M_{1}: M_{2}: M_{3}$ will not be in the order $1: \sim 2: \sim 7$ as in mSUGRA. Although the $\widetilde{Z}_{2}-\widetilde{Z}_{1}$ mass edge will be directly measurable, the absolute neutralino and chargino masses will be difficult to extract at the LHC. In frame $d$ ), we show the spectrum from BWCA3, which is similar to the case of BWCA2. 

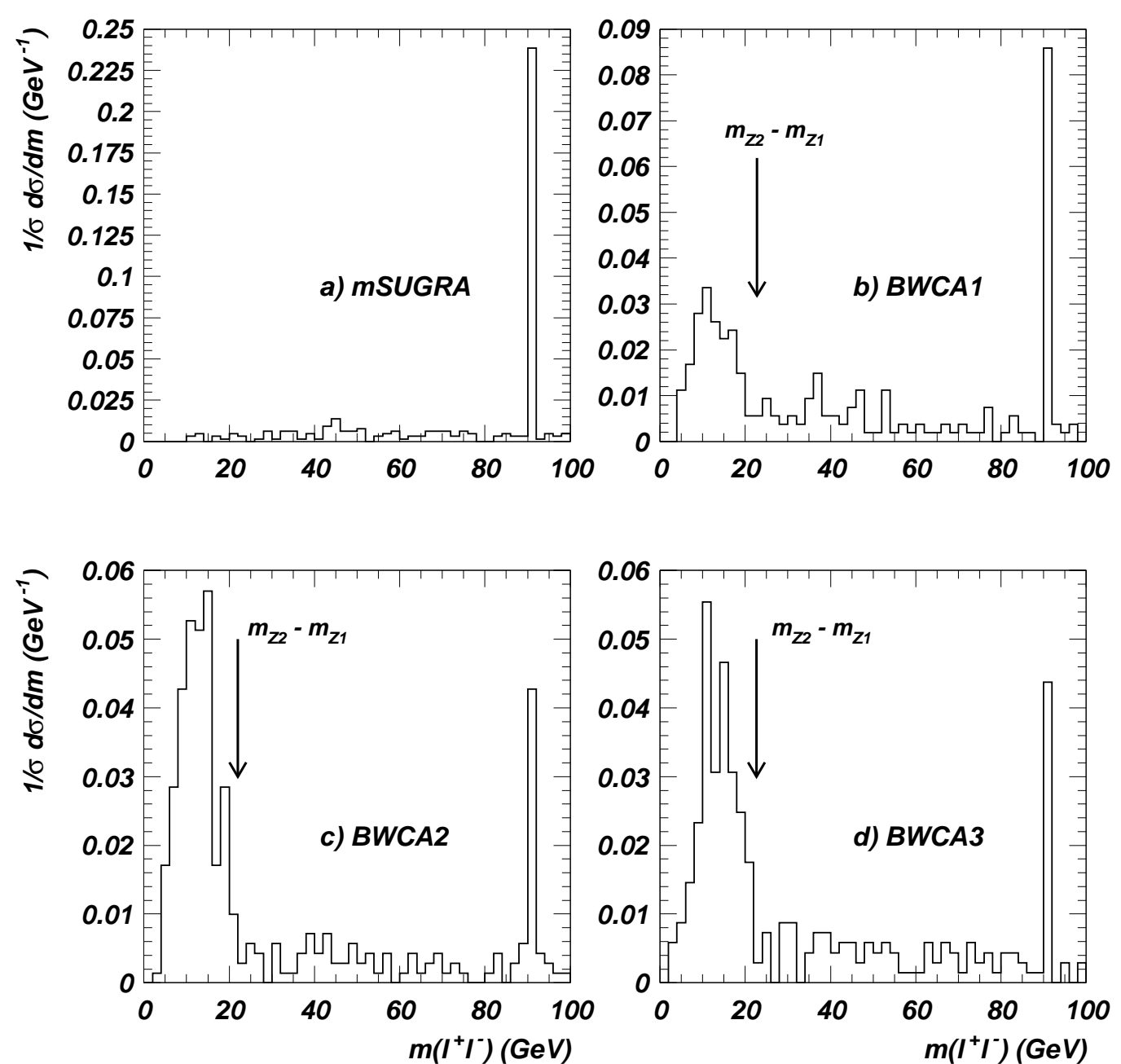

Figure 17: Distribution of same flavor/opposite sign dileptons from SUSY events at the CERN LHC from $a$ ) mSUGRA, $b$ ) BWCA1, $c$ ) BWCA2 and $d$ ) BWCA3 cases as in table 2 .

\subsection{Linear $e^{+} e^{-}$collider}

The reach of the CERN LHC for supersymmetric matter is determined mainly by $m_{\tilde{q}}$ and $m_{\tilde{g}}$, which depend on $m_{0}$ and $m_{1 / 2}$. In contrast, the reach of the ILC for SUSY is largely determined by whether or not the reactions $e^{+} e^{-} \rightarrow \widetilde{W}_{1}^{+} \widetilde{W}_{1}^{-}$or $e^{+} e^{-} \rightarrow \tilde{\ell}^{+} \tilde{\ell}^{-}$ are kinematically accessible 92]. For instance, chargino pair production is expected to be visible if $\sqrt{s}>2 m_{\widetilde{W}_{1}}$. The value of $m_{\widetilde{W}_{1}}$ depends mainly on $M_{2}$ and $\mu$. Thus, in the BWCA case where $M_{2}=m_{1 / 2}$ but $M_{1}$ is variable, the reach of the ILC in the $m_{0}$ vs. $m_{1 / 2}$ plane will be similar to the case of the mSUGRA model. However, in the BWCA case where $M_{1}=m_{1 / 2}$, with variable $M_{2}$, the reach of the ILC will be enhanced compared to the mSUGRA case, since $\left|M_{2}\right|$ is typically much smaller for a given set of $m_{0}$ and $m_{1 / 2}$ values. The situation is illustrated in figure 18 where we show the ultimate reach of the LHC and the ILC in the $m_{0}$ vs. $m_{1 / 2}$ plane for $\tan \beta=10, A_{0}=0, \mu>0$ and $m_{t}=178 \mathrm{GeV}$. We have dialed $-M_{2}$ at every point to give $\Omega_{\widetilde{Z}_{1}} h^{2}=0.11$, in accord with the WMAP observation. We have assumed $100 \mathrm{fb}^{-1}$ of integrated luminosity for both 


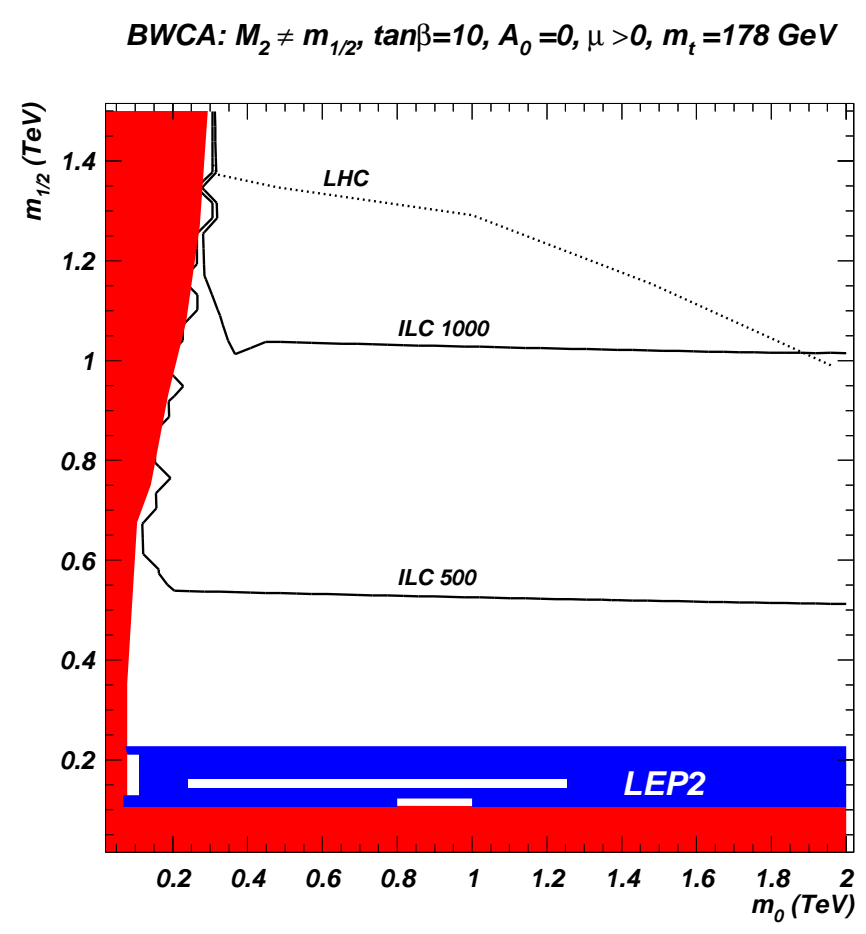

Figure 18: Reach of the ILC and the LHC for SUSY in the BWCA DM scenario where $\left|M_{2}\right|$ is lowered until $\Omega_{\widetilde{Z}_{1}} h^{2}=0.11$ at every point in the $m_{0} v s . m_{1 / 2}$ plane. We assume $100 \mathrm{fb}^{-1}$ of integrated luminosity for each collider, and show the ILC reach for $\sqrt{s}=500 \mathrm{GeV}$ and $1000 \mathrm{GeV}$.

LHC and ILC. The reach of ILC with $\sqrt{s}=500 \mathrm{GeV}$ (denoted by ILC 500) extends to $m_{1 / 2} \sim 500 \mathrm{GeV}$, while the corresponding reach in the mSUGRA model with gaugino mass unification extends to $m_{1 / 2} \sim 320 \mathrm{GeV}$ [92]. The reach of ILC with $\sqrt{s}=1000 \mathrm{GeV}$ extends to $m_{1 / 2} \sim 1 \mathrm{TeV}$, compared with the mSUGRA value of $m_{1 / 2} \sim 600 \mathrm{GeV}$. In fact, we see that for $m_{0} \gtrsim 1.9 \mathrm{TeV}$, the ILC1000 reach begins to exceed that of the LHC. In this region, $m_{\widetilde{W}_{1}} \sim 500 \mathrm{GeV}$, while $m_{\tilde{g}} \sim 2300 \mathrm{GeV}$ and $m_{\tilde{q}} \sim 2675 \mathrm{GeV}$.

At a $\sqrt{s}=500 \mathrm{GeV}$ ILC, the new physics reactions for the four case studies shown in table 2 would include $Z h, \widetilde{W}_{1}^{+} \widetilde{W}_{1}^{-}, \widetilde{Z}_{1} \widetilde{Z}_{2}$ and $\widetilde{Z}_{2} \widetilde{Z}_{2}$ production. It was shown in ref. 92. that even in the case of a small $\widetilde{W}_{1}-\widetilde{Z}_{1}$ mass gap chargino pair production events could still be identified above SM backgrounds using cuts specially designed to pick out low visible energy acollinear signal events over backgrounds from $e^{+} e^{-}$and $\gamma \gamma$ processes. The chargino and neutralino masses can be inferred from the resultant dijet distribution in $\widetilde{W}_{1}^{+} \widetilde{W}_{1}^{-} \rightarrow\left(\bar{\ell} \nu_{\ell} \widetilde{Z}_{1}\right)+\left(q \bar{q} \widetilde{Z}_{1}\right)$ events [93, 94, 92]. These measurements should allow the absolute mass scale of the sparticles to be pinned down, and will complement the $\widetilde{Z}_{2}-\widetilde{Z}_{1}$ mass gap measurement from the CERN LHC. The combination of $m_{\widetilde{Z}_{2}}, m_{\widetilde{W}_{1}}, m_{\widetilde{Z}_{1}}$ and $m_{\widetilde{Z}_{2}}-m_{\widetilde{Z}_{1}}$ measurements will point to whether or not gaugino mass unification is realized in nature.

The dependence of the cross sections for $\widetilde{W}_{1}^{+} \widetilde{W}_{1}^{-}, \widetilde{Z}_{1} \widetilde{Z}_{2}$ and $\widetilde{Z}_{2} \widetilde{Z}_{2}$ production on the longitudinal polarization of the electron beam provides an additional tool at the ILC. This dependence is illustrated in figure 19 for the mSUGRA model in table 2 in frame $a$ ), for case 

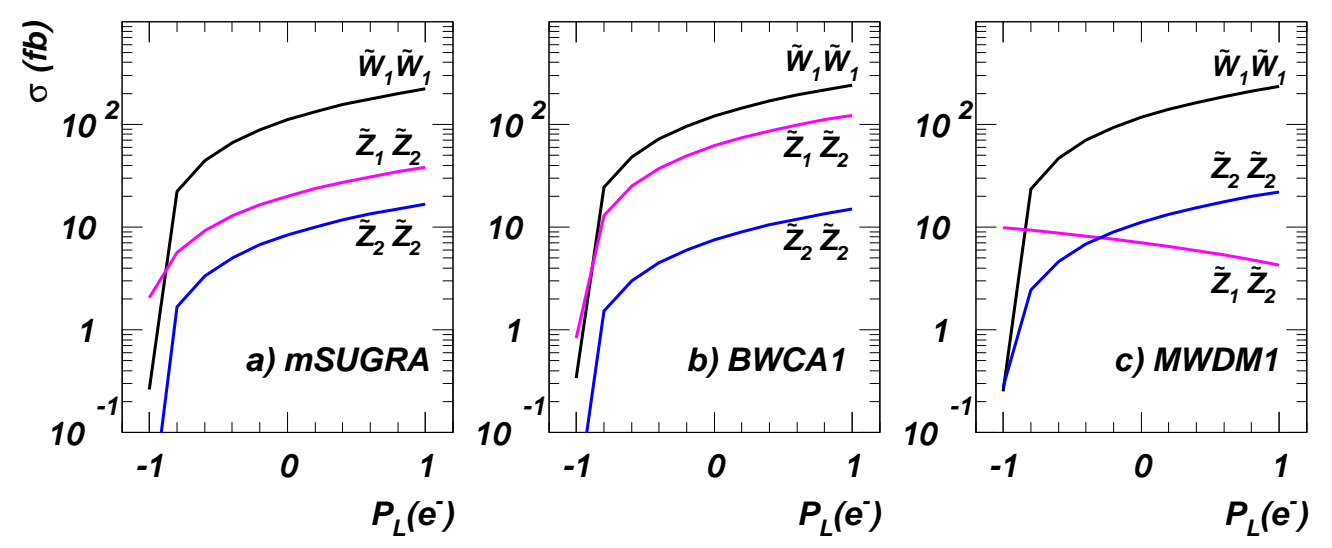

Figure 19: Cross sections for $e^{+} e^{-} \rightarrow \widetilde{W}_{1}^{+} \widetilde{W}_{1}^{-}, \widetilde{Z}_{1} \widetilde{Z}_{2}$ and $\widetilde{Z}_{2} \widetilde{Z}_{2}$ processes versus electron beam polarization $P_{L}\left(e^{-}\right)$for an electron-positron linear collider operating at $\sqrt{s}=500 \mathrm{GeV}$ for $a$ ) mSUGRA, $b$ ) BWCA1 and $c$ ) MWDM1 from ref. [24]. The positron beam is taken to be unpolarized.

BWCA1 in frame $b$ ) and for case MWDM1, which is point 1 of ref. [24]. In all three cases, the chargino and the second lightest neutralino have dominant wino components so that the magnitudes and the polarization depence of $\sigma\left(\widetilde{W}_{1} \widetilde{W}_{1}\right)$ and $\sigma\left(\widetilde{Z}_{2} \widetilde{Z}_{2}\right)$ are qualitatively similar to one another and to the corresponding dependence in the mSUGRA model [94]. A minor difference is that, for $P_{L}=-1, \sigma\left(\widetilde{Z}_{2} \widetilde{Z}_{2}\right)$ does not fall to quite as small values for the MWDM1 case because its hypercharge gaugino component always remains significant, as we have already discussed. The polarization-dependence and/or the magnitude of the $e^{+} e^{-} \rightarrow \widetilde{Z}_{1} \widetilde{Z}_{2}$ process are, however, quite different in the three cases. In the limit that $\widetilde{Z}_{1}$ is a bino and $\widetilde{Z}_{2}$ is the wino (a good approximation in the mSUGRA case and an even better approximation in the BWCA case), $t$-channel selectron exchange dominates the amplitude. ${ }^{10}$ Recall, however, that for $P_{L}\left(e^{-}\right)=1\left(P_{L}\left(e^{-}\right)=-1\right)$ only $\tilde{e}_{L}\left(\tilde{e}_{R}\right)$ exchange is possible. Since the chargino is always wino-like, its couplings to $\tilde{e}_{R}$ are strongly suppressed, accounting for the behaviour of the cross sections in the first two frames. For the MWDM1 case in frame $c$ ), we would naively expect that $\widetilde{Z}_{1}$ would be photino-like and $\widetilde{Z}_{2}$ would be zino-like. It turns out, however, that (for the specific MWDM1 parameters) the difference $M_{1}$ (weak) $-M_{2}$ (weak) is sufficiently large, and results in a flip of the relative sign between the gaugino components of both $\widetilde{Z}_{1}$ and $\widetilde{Z}_{2}$, while roughly preserving the magnitude. This strongly suppresses the $e \tilde{e}_{L} \widetilde{Z}_{1}$ coupling, and hence, the $\tilde{e}_{L}$ exchange amplitude, resulting in the relatively flat and even decreasing polarization-dependence of the cross section. ${ }^{11}$

A very striking feature of the figure is the very large cross section for $\widetilde{Z}_{1} \widetilde{Z}_{2}$ production for a left polarized electron beam for the BWCA1 case in frame $b$ ), as compared with the mSUGRA case. In both cases, since $\widetilde{Z}_{1}$ and $\widetilde{Z}_{2}$ couple to (a not-so-heavy) $\tilde{e}_{L}$ mainly via their large hypercharge and $\mathrm{SU}(2)$ gaugino components, respectively, $\tilde{e}_{L}$ exchange completely dominates $\widetilde{Z}_{1} \widetilde{Z}_{2}$ production at $P_{L}=1$. Moreover, the magnitudes of

\footnotetext{
${ }^{10}$ Recall that $Z$ couples to neutralinos only via their suppressed higgsino components.

${ }^{11}$ When both $\tilde{e}_{L}$ and $\tilde{e}_{R}$ exchanges are dynamically suppressed, the effect of the usually small $Z$ exchange contribution (which leads to a more or less flat polarization dependence) may also be significant.
} 
the couplings, as well as selectron masses, are very comparable in the two scenarios. The reason for the difference in the cross sections lies in the relative sign between the $\widetilde{Z}_{1}$ and $\widetilde{Z}_{2}$ eigenvalues (not physical masses) of the neutralino mass matrix. Since $M_{1} / M_{2}<0$, we expect this sign to flip in the BWCA case as compared with the mSUGRA or the MWDM cases, where $M_{1} / M_{2}$ is positive. This is relevant because when we square the $t$-channel amplitude and sum over the neutralino spins, there is one term that is proportional to the product $m_{\widetilde{Z}_{1}} m_{\widetilde{Z}_{2}}$ (see e.g. eq. (8f) of ref. [95], where the last term that includes the factor $(-1)^{\theta_{i}+\theta_{j}}$ is the one we are referring to). This term always flips sign between any scenario with positive $M_{1} / M_{2}$ vs. negative $M_{1} / M_{2}$. As a result, what was a significant cancellation in the mSUGRA case in frame $a$ ) of figure 19 turns into a sum in frame $b$ ), accounting for the factor $\sim 2.5$ increase in $\sigma\left(\widetilde{Z}_{1} \widetilde{Z}_{2}\right)$ at $P_{L}=1$.

We examine this potential enhancement of the $e^{+} e^{-} \rightarrow \widetilde{Z}_{1} \widetilde{Z}_{2}$ production cross section at a $\sqrt{s}=0.5 \mathrm{TeV}$ linear collider in figure 20. In frame $a)$, we show contours of $\sigma\left(e^{+} e^{-} \rightarrow\right.$ $\left.\widetilde{Z}_{1} \widetilde{Z}_{2}\right)$ in $\mathrm{fb}$ in the $m_{0}$ vs. $m_{1 / 2}$ plane for $A_{0}=0, \tan \beta=10$ and $\mu>0$, in the case of the mSUGRA model. The light (yellow) shaded region is where either chargino pair production or selectron pair production is kinematically accessible at a $\sqrt{s}=0.5 \mathrm{TeV}$ ILC. We see that the $\widetilde{Z}_{1} \widetilde{Z}_{2}$ cross section only exceeds the $100 \mathrm{fb}$ level at the very lowest values of $m_{0}$ and $m_{1 / 2}$, in the lower left corner. The cross section at the $\sim 10 \mathrm{fb}$ level gives some additional reach of an ILC for SUSY beyond the range where chargino pair production or slepton pair production are possible [94, 92]. In frame $b$ ), we show the same situation for the BWCA scenario where $M_{1}$ has been set to negative values everywhere in the $m_{0}$ vs. $m_{1 / 2}$ plane such that the WMAP value $\Omega_{\widetilde{Z}_{1}} h^{2}=0.11$ is fulfilled. In this case, the $\widetilde{Z}_{1} \widetilde{Z}_{2}$ production cross section is in general increased everywhere, and exceeds $100 \mathrm{fb}$ in a much larger region. Of course, the region of reaction kinematic accessibility has decreased somewhat due to the increase in $m_{\widetilde{Z}_{1}}$ for a given $m_{0}$ and $m_{1 / 2}$ value, so that contours of accessibility reach only up to $m_{1 / 2} \sim 350 \mathrm{GeV}$ (as opposed to $420 \mathrm{GeV}$ in the mSUGRA case). Also, the cross section falls off at large values of $m_{0}$ since the amplitude is suppressed with increasing selectron mass. In frame $c$ ), we show the same cross section in the $m_{0} v s . m_{1 / 2}$ plane, except here $M_{2}$ is taken negative, and is decreased at each point in absolute value until the WMAP value is obtained. In this case, the kinematically accessible region increases since for a given $m_{0}$ and $m_{1 / 2}$ value $m_{\widetilde{Z}_{2}}$ is lowered compared to the mSUGRA case, while $m_{\widetilde{Z}_{1}}$ stays fixed. Again, a rather large region appears with $\sigma>10-100 \mathrm{fb}$, so that $\widetilde{Z}_{1} \widetilde{Z}_{2}$ production should be robust over much of parameter space in the BWCA scenario as long as $\widetilde{Z}_{1} \widetilde{Z}_{2}$ production is kinematically accessible and $m_{0}$ is not too large. An unexpectedly large value of $\sigma\left(\widetilde{Z}_{1} \widetilde{Z}_{2}\right) / \sigma\left(\widetilde{W}_{1} \widetilde{W}_{1}\right)$ may, therefore, be an indication that $M_{1} / M_{2}<0$ and that the selectrons are not too heavy.

The rather large $e^{+} e^{-} \rightarrow \widetilde{Z}_{1} \widetilde{Z}_{2}$ cross section at the ILC potentially leads to another distinguishing feature of SUSY events at the ILC in the BWCA model: at least for $m_{0} \lesssim$ $1 \mathrm{TeV}$, we should expect a large rate for events with one or more isolated photons due to the enhanced branching fraction for $\widetilde{Z}_{2} \rightarrow \widetilde{Z}_{1} \gamma$ decays. Thus, from $\widetilde{Z}_{1} \widetilde{Z}_{2}$ production, we may expect $\gamma+\not E$ events, while from $\widetilde{Z}_{2} \widetilde{Z}_{2}$ production, we may expect $\gamma \gamma+\not E, \gamma+j e t s+\not E$ events, $\gamma+\ell \bar{\ell}+E$ events and $\gamma+E$. Since the $\gamma$ arises from a two body decay, the endpoints of the $E(\gamma)$ distribution will be functions of $\sqrt{s}, m_{\widetilde{Z}_{2}}$ and $m_{\widetilde{Z}_{1}}$. A measurement 

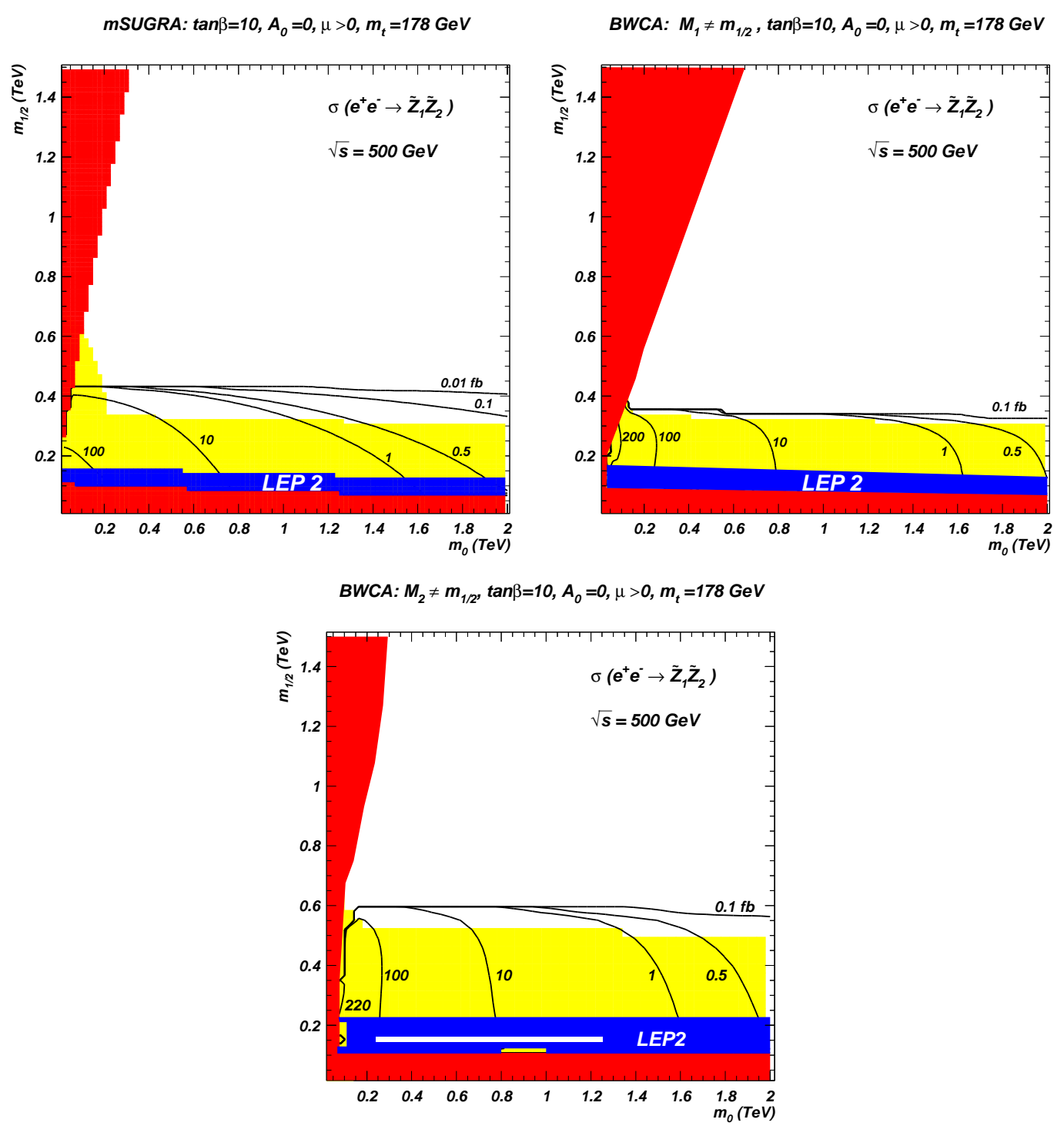

Figure 20: Contours of $\sigma\left(e^{+} e^{-} \rightarrow \widetilde{Z}_{1} \widetilde{Z}_{2}\right)$ in fb for a $\sqrt{s}=0.5 \mathrm{TeV}$ ILC with unpolarized beams in the $m_{0}$ vs. $m_{1 / 2}$ plane for $\tan \beta=10, A_{0}=0, \mu>0$ and $a$ ) mSUGRA model, $\left.b\right)-M_{1}>m_{1 / 2}$ BWCA and $c)-M_{2}<m_{1 / 2}$ BWCA. The yellow shaded regions show where $\widetilde{W}_{1}^{+} \widetilde{W}_{1}^{-}$and/or $\tilde{\ell}^{+} \tilde{\ell}^{-}$ should also be kinematically accessible to a $\sqrt{s}=0.5 \mathrm{TeV}$ ILC.

of the endpoints of this distribution will thus allow an independent measurement of the two lighter neutralino masses. The $E(\gamma)$ distribution is shown in figure 21 for BWCA2 at $\mathrm{a} \sqrt{s}=0.5 \mathrm{TeV}$ ILC.

\section{Conclusions}

In this paper, we have considered the phenomenological implications of neutralino dark matter in the bino-wino co-annihilation (BWCA) scenario, and compared these to the case of mixed wino dark matter which has a qualitatively similar spectrum. The BWCA 
scenario arises in models with non-universal gaugino masses where, at the weak scale, $\left|M_{1}\right| \sim\left|M_{2}\right|$, but where the two gauginos masses have opposite signs. The same sign case gives rise to mixed wino dark matter, while in the BWCA case, there is little mixing so that the $\widetilde{Z}_{1}$ remains nearly a pure bino, while $\widetilde{Z}_{2}$ remains nearly a pure wino. The scenario can be brought into accord with the WMAP $\Omega_{\widetilde{Z}_{1}} h^{2} \sim 0.11$ constraint by arranging for $m_{\widetilde{W}_{1}} \sim m_{\widetilde{Z}_{2}} \sim m_{\widetilde{Z}_{1}}$, so that bino-wino co-annihilation is the dominant neutralino annihilation mechanism in the early universe.

Since co-annihilation processes are the dominant mechanism for the annihilation of relic neutralinos from the Big Bang, it is expected that indirect dark matter detection rates (which depend on the $\widetilde{Z}_{1} \widetilde{Z}_{1}$ pair-annihilation cross section) will typically be quite low in the BWCA scenario. Direct detection of neutralinos may be possible for BWCA DM at stage 3 detectors in some portions of parameter space. In contrast, for the MWDM case, neutralino annihilation cross sections are enhanced relative to mSUGRA so that it is more likely that an indirect signal for DM will be seen. If we already have some infomation about the sparticle spectrum from the LHC, it may be that results from direct and indirect $\mathrm{DM}$ detection experiments may serve

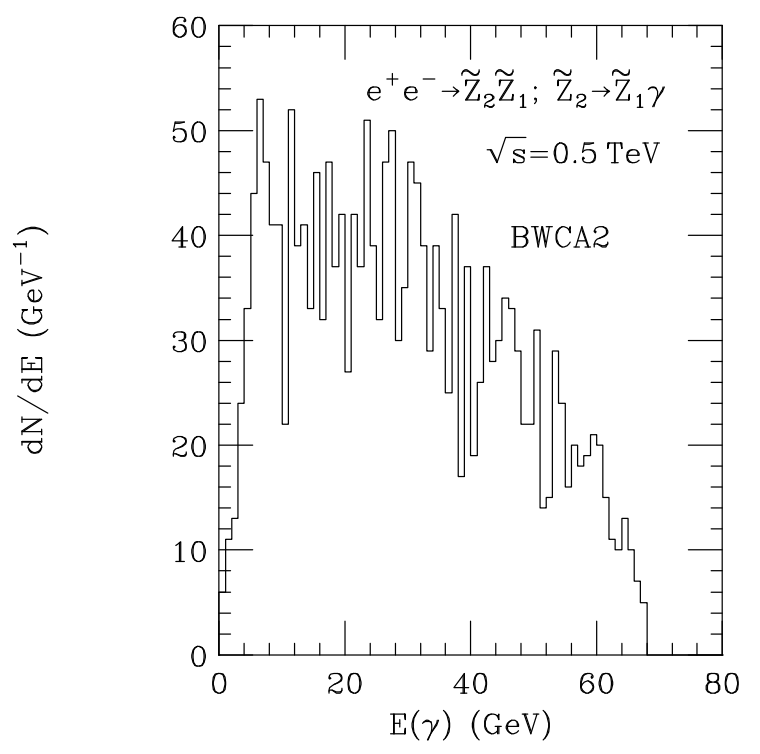

Figure 21: Distribution in $E(\gamma)$ from $e^{+} e^{-} \rightarrow$ $\widetilde{Z}_{2} \widetilde{Z}_{1}$ with $\widetilde{Z}_{2} \rightarrow \gamma \widetilde{Z}_{1}$ for point BWCA2 in $e^{+} e^{-}$ collisions at $\sqrt{s}=0.5 \mathrm{TeV}$.

to discriminate between these scenarios.

The small mass gap expected between $m_{\widetilde{Z}_{2}}$ and $m_{\widetilde{Z}_{1}}$ is a hallmark of both the BWCA and the MWDM scenarios, and leads to a variety of interesting consequences for collider experiments. In teh BWCA case, if $m_{0} \lesssim 1 \mathrm{TeV}$, then the radiative decay $\widetilde{Z}_{2} \rightarrow \widetilde{Z}_{1} \gamma$ is greatly enhanced, leading to the production of isolated photons at the Tevatron, LHC and ILC. While extraction of the isolated photon signal above SM background looks difficult at the Tevatron, they should yield observable signals at the CERN LHC. The LHC should also be able to extract the $m_{\widetilde{Z}_{2}}-m_{\widetilde{Z}_{1}}$ mass difference from gluino and squark cascade decay events which contain isolated opposite sign/same flavor dilepton pairs. By comparing the neutralino mass difference to measurements of the gluino mass, we should readily be able to infer that the weak scale gaugino masses are incompatible with expectation from models with a universal gaugino mass at the high scale. At the ILC, again small $m_{\widetilde{W}_{1}}-m_{\widetilde{Z}_{1}}$ and $m_{\widetilde{Z}_{2}}-m_{\widetilde{Z}_{1}}$ mass gaps should be measurable, as in the MWDM and MHDM scenarios. It should also be possible to extract the weak scale values of the gaugino mass parameters. However, in the case of BWCA, $\widetilde{Z}_{1} \widetilde{Z}_{2}$ production should occur at enhanced rates relative to mSUGRA and MWDM, with a production cross section which increases with $P_{L}\left(e^{-}\right)$. In constrast to MHDM, the $\widetilde{Z}_{3}$ and $\widetilde{Z}_{4}$ states will typically occur at much higher mass scales associated with large values for the $|\mu|$ parameter. 


\section{Acknowledgments}

We thank K. Melnikov for the idea of using effective theories in quantum mechanics, as discussed in the appendix. This research was supported in part by the U.S. Department of Energy grant numbers DE-FG02-97ER41022, DE-FG03-94ER40833. T. K. was supported in part by the U.S. Department of Energy under contract No. DE-AC02-98CH10886.

\section{A. Effective theories in quantum mechanics}

Consider a situation where the total hamiltonian can be split into two pieces,

$$
H=H_{0}+V,
$$

so that the spectrum of $H_{0}$ is hierarchical, i.e., it consists of "low energy" states $\left|l_{i}\right\rangle$, possibly interacting with one another via interactions included in $H_{0}$, and "high energy" states, $\left|h_{a}\right\rangle$ (again possibly interacting with themselves via interactions included in $H_{0}$ ). Without loss of generality, all interactions between the states $\left\{\left|l_{i}\right\rangle\right\}$ and the states $\left\{\left|h_{a}\right\rangle\right\}$ are encapsulated in $V$. In other words, $\left\langle h_{a}\left|H_{0}\right| l_{i}\right\rangle=0$, and $\left\langle l_{i}|V| l_{j}\right\rangle=\left\langle h_{a}|V| h_{b}\right\rangle=0$.

We seek a description of low energy states in terms of an effective hamiltonian $H_{\text {eff }}$, that acts only on the low energy states $\left\{\left|l_{i}\right\rangle\right\}$, assuming that we are at energies that are too low to excite the high energy states. Note that even if $H_{0}$ is completely diagonal in the low energy sector, we would expect that scattering of low energy states (with energy $E \ll M_{a}$ ) would occur via their interactions with the high energy sector, with an amplitude suppressed by $E / M_{a}$. Here, we show how this comes about and obtain an expression for $H_{\text {eff. }}$.

We define $H_{\text {eff }}$ by matching the low energy Green's functions of the full theory with those of the effective theory,

$$
\left\langle l_{i}\left|\frac{1}{H_{0}+V-z}\right| l_{j}\right\rangle \equiv\left\langle l_{i}\left|\frac{1}{H_{\mathrm{eff}}-z}\right| l_{j}\right\rangle, \quad \text { if } \quad|z| \ll M_{a} .
$$

Here, $z$ is the complex argument of the Green's function. Expanding the left hand side of (A.1), we obtain

$$
\begin{aligned}
\left\langle l_{i}\left|\frac{1}{H_{\mathrm{eff}}-z}\right| l_{j}\right\rangle= & \left\langle l_{i}\left|\frac{1}{H_{0}-z}\right| l_{j}\right\rangle+\left\langle l_{i}\left|\frac{1}{H_{0}-z}(-V) \frac{1}{H_{0}-z}\right| l_{j}\right\rangle+ \\
& +\left\langle l_{i}\left|\frac{1}{H_{0}-z}(-V) \frac{1}{H_{0}-z}(-V) \frac{1}{H_{0}-z}\right| l_{j}\right\rangle+\cdots .
\end{aligned}
$$

Clearly, if $V=0$ (no interactions between low and high energy states), $H_{\text {eff }}$ is just $H_{0}$ restricted to the low energy subspace, i.e. $H_{\text {eff }}=H_{0} \mathcal{P}_{\text {low }}$, where $\mathcal{P}_{\text {low }}$ is the projector on to the low energy subspace. It is also clear that terms with an odd number of $(-V)$ factors on the right hand side of (A.2) are all zero because $V$ only connects states in the low energy sector with those in the high energy sector, whereas $H_{0}$ only connects low energy (high energy) states with one another. The third term on the right hand side of (A.2) can be written as

$$
\left(\frac{1}{H_{0}-z}\right)_{i i^{\prime}}(-V)_{i^{\prime} a}\left(\frac{1}{H_{0}-z}\right)_{a b}(-V)_{b j^{\prime}}\left(\frac{1}{H_{0}-z}\right)_{j^{\prime} j},
$$


with the repeated indices $i^{\prime}, a, b$ and $j^{\prime}$ all summed up. We now defined an induced effective potential $V^{\text {induced }}$ by,

$$
-\left(V^{\text {induced }}\right)_{i^{\prime} j^{\prime}}=(-V)_{i^{\prime} a}\left(\frac{1}{H_{0}-z}\right)_{a b}(-V)_{b j^{\prime}}
$$

in terms of which this term can be written as,

$$
\left(\frac{1}{H_{0}-z}\right)_{i i^{\prime}}\left(-V^{\text {induced }}\right)_{i^{\prime} j^{\prime}}\left(\frac{1}{H_{0}-z}\right)_{j^{\prime} j} .
$$

In exactly the same manner, the term with four factors of $(-V)$ will end up as one with two factors of $\left(-V^{\text {induced }}\right)$, etc. so that we have a geometric series that can be summed to give

$$
\left\langle l_{i}\left|\frac{1}{H_{\mathrm{eff}}-z}\right| l_{j}\right\rangle=\left\langle l_{i}\left|\frac{1}{H_{0}+V^{\text {induced }}-z}\right| l_{j}\right\rangle,
$$

showing that the interactions between the low and high sectors effectively induce an additional "potential" in the low sector.

Up to now, our considerations (though formal) have been "exact" in the sense that we have not made any low energy approximation. This shows up in the fact that the "potential" $V^{\text {induced }}$ defined in (A.3) depends on the "energy" $z$. If $|z|$ is small compared with the high energy scale associated with the spectrum of $H_{0}$, we can ignore it in the evaluation of the matrix elements of $\left(H_{0}-z\right)^{-1}$ between high energy states, and approximate $V^{\text {induced }}$ by,

$$
\begin{aligned}
\left(V^{\text {induced }}\right)_{i j} & =-\sum_{a, b}\left[V_{i a}\left(\frac{1}{H_{0}}\right)_{a b} V_{b j}+V_{i a} \mathcal{O}\left(\frac{|z|}{M_{a}^{2}}\right) V_{b j}+\cdots\right] \\
& \simeq-\sum_{a, b} V_{i a}\left(\frac{1}{H_{0}}\right)_{a b} V_{b j}
\end{aligned}
$$

where, in the last step, we have made the "low energy approximation" and obtained what is a conventional potential (independent of $z$ ). Assuming that the matrix elements $V_{i a}$ have magnitudes corresponding to the low energy scale, we see that the $\left(\frac{1}{H_{0}}\right)_{a b}$ term supresses the low energy matrix elements of $V^{\text {induced }}$ by $|z| / M_{a}$ as expected.

This expression is particularly useful in two cases.

1. If $H_{0} \mathcal{P}_{\text {low }}$ is diagonal, then all interactions arise only from $V^{\text {induced }}$ and this analysis is essential to obtain any scattering in the low energy sector.

2. If the low energy sector has an approximate symmetry that is violated only by its interactions with the high mass sector, or even just by interactions solely within the high mass sector, we can use $V^{\text {induced }}$ to study these symmetry violations. 
In the analysis up to now, we have retained just the leading correction in powers of $M_{a}$. It is straightforward to retain the $\mathcal{O}\left(1 / M_{a}^{2}\right)$ term in the expansion of the Green's function $\left(H_{\text {eff }}-z\right)^{-1}$. The energy eigenvalues $E$ in the low energy theory are given by those values of $z$ where the corresponding Green's function develops a singularity,i.e., where

$$
H_{\mathrm{eff}}|\psi\rangle=E|\psi\rangle \text {. }
$$

Assuming, for simplicity, that $H_{0}$ is diagonal in the high mass sector and retaining terms to $\mathcal{O}\left(1 / M_{a}^{2}\right)$, we find that these eigenvalues are given by the generalized matrix eigenvalue equation,

$$
\sum_{j}\left[\left(H_{0}\right)_{i j}-\sum_{a} V_{i a} \frac{1}{M_{a}} V_{a j}\right] c_{j}=E \sum_{j}\left[\delta_{i j}+\sum_{a} V_{i a} \frac{1}{M_{a}^{2}} V_{a j}\right] c_{j} .
$$

The positive operator on the right hand side serves as a metric in the low energy subspace.

This formalism can be directly used to obtain the eigenvalues (2.9) of the neutralino mas matrix in the large $|\mu|$ limit. In this case, the low energy sector comprises of the neutral wino and the bino, and the generalized eigenvalue equation takes the form,

$$
\left(\begin{array}{cc}
M_{2}+a & -a \tan \theta_{W} \\
-a \tan \theta_{W} & M_{1}+a \tan ^{2} \theta_{W}
\end{array}\right)\left(\begin{array}{l}
c_{1} \\
c_{2}
\end{array}\right)=E\left(\begin{array}{cc}
1+\frac{M_{W}^{2}}{\mu^{2}} & -\frac{M_{W}^{2}}{\mu^{2}} \tan \theta_{W} \\
-\frac{M_{W}^{2}}{\mu^{2}} \tan \theta_{W} & 1+\frac{M_{W}^{2}}{\mu^{2}} \tan ^{2} \theta_{W}
\end{array}\right)\left(\begin{array}{l}
c_{1} \\
c_{2}
\end{array}\right),
$$

with $a \equiv \frac{M_{W}^{2}}{\mu} \sin 2 \beta$ much smaller than $M_{1}$ and $M_{2}$ in the large $|\mu|$ limit.

\section{References}

[1] For recent reviews, see e.g. C. Jungman, M. Kamionkowski and K. Griest, Supersymmetric dark matter, Phys. Rept. 267 (1996) 195;

A. Lahanas, N. Mavromatos and D. Nanopoulos, WMAPing the universe: supersymmetry, dark matter, dark energy, proton decay and collider physics, Int. J. Mod. Phys. D 12 (2003) 1529 hep-ph/0308251;

M. Drees, Supersymmetric dark matter 2004, hep-ph/0410113;

K.A. Olive, Tasi lectures on astroparticle physics, astro-ph/0503065.

[2] P. Gondolo, J. Edsjo, P. Ullio, L. Bergstrom, M. Schelke and E. A. Baltz, DarkSUSY: computing supersymmetric dark matter properties numerically, JCAP 0407 (2004) 008 astro-ph/0406204:

G. Belanger, F. Boudjema, A. Pukhov and A. Semenov, Micromegas: a program for calculating the relic density in the MSSM, Comput. Phys. Commun. 149 (2002) 103 hep-ph/0112278.

[3] H. Baer, C. Balazs and A. Belyaev, Neutralino relic density in minimal supergravity with co-annihilations, JHEP 03 (2002) 042 hep-ph/0202076.

[4] WMAP collaboration, D.N. Spergel et al., First year Wilkinson microwave anisotropy probe (wmap) observations: determination of cosmological parameters, Astrophys. J. Suppl. 148 (2003) 175 astro-ph/0302209;

C.L. Bennett et al., First year Wilkinson microwave anisotropy probe (WMAP) observations: preliminary maps and basic results, Astrophys. J. Suppl. 148 (2003) 1 astro-ph/0302207. 
[5] J.R. Ellis, K.A. Olive, Y. Santoso and V.C. Spanos, Supersymmetric dark matter in light of WMAP, Phys. Lett. B 565 (2003) 176 hep-ph/0303043;

$\mathrm{H}$. Baer and C. Balazs, $\chi^{2}$ analysis of the minimal supergravity model including WMAP, $(g-2)_{\mu}, b \rightarrow s \gamma$ constraints, JCAP 05 (2003) 006;

U. Chattopadhyay, A. Corsetti and P. Nath, WMAP constraints, SUSY dark matter and implications for the direct detection of SUSY, Phys. Rev. D 68 (2003) 035005 hep-ph/0303201;

A.B. Lahanas and D.V. Nanopoulos, WMAPing out supersymmetric dark matter and phenomenology, Phys. Lett. B 568 (2003) 55 hep-ph/0303130;

for a review, see A. Lahanas, N. Mavromatos and D. Nanopoulos, WMAPing the universe: supersymmetry, dark matter, dark energy, proton decay and collider physics, Int. J. Mod. Phys. D 12 (2003) 1529.

[6] A.H. Chamseddine, R. Arnowitt and P. Nath, Locally supersymmetric grand unification, Phys. Rev. Lett. 49 (1982) 970:

R. Barbieri, S. Ferrara and C.A. Savoy, Gauge models with spontaneously broken local supersymmetry, Phys. Lett. B 119 (1982) 343;

N. Ohta, Grand unified theories based on local supersymmetry, Prog. Theor. Phys. 70 (1983) 542 ;

L.J. Hall, J.D. Lykken and S. Weinberg, Supergravity as the messenger of supersymmetry breaking, Phys. Rev. D 27 (1983) 2359;

for reviews, see H.P. Nilles, Supersymmetry, supergravity and particle physics, Phys. Rept. 110 (1984) 1 and P. Nath, Twenty years of SUGRA, hep-ph/0307123.

[7] H. Goldberg, Constraint on the photino mass from cosmology, Phys. Rev. Lett. 50 (1983) 1419 ;

J. Ellis, J. Hagelin, D. Nanopoulos and M. Srednicki, Search for supersymmetry at the $\bar{p} p$ collider, Phys. Lett. B 127 (1983) 233;

J. Ellis, J. Hagelin, D. Nanopoulos, K. Olive and M. Srednicki, Supersymmetric relics from the big bang, Nucl. Phys. B 238 (1984) 453.

[8] H. Baer and M. Brhlik, Cosmological relic density from minimal supergravity with implications for collider physics, Phys. Rev. D 53 (1996) 597 hep-ph/9508321;

V.D. Barger and C. Kao, Relic density of neutralino dark matter in supergravity models, Phys. Rev. D 57 (1998) 3131 hep-ph/9704403.

[9] J.R. Ellis, T. Falk and K.A. Olive, Neutralino stau coannihilation and the cosmological upper limit on the mass of the lightest supersymmetric particle, Phys. Lett. B 444 (1998) 367 hep-ph/9810360;

J. Ellis, T. Falk, K. Olive and M. Srednicki, Calculations of neutralino-stau coannihilation channels and the cosmologically relevant region of MSSM parameter space, Astropart. Phys. 13 (2000) 181 hep-ph/9905481;

M.E. Gómez, G. Lazarides and C. Pallis, Supersymmetric cold dark matter with Yukawa unification, Phys. Rev. D 61 (2000) 123512 hep-ph/9907261;

M.E. Gomez, G. Lazarides and C. Pallis, Yukawa unification, $b \rightarrow s \gamma$ and bino stau coannihilation, Phys. Lett. B 487 (2000) 313 hep-ph/0004028;

A.B. Lahanas, D.V. Nanopoulos and V.C. Spanos, Neutralino relic density in a universe with non-vanishing cosmological constant, Phys. Rev. D 62 (2000) 023515 hep-ph/9909497;

R. Arnowitt, B. Dutta and Y. Santoso, Coannihilation effects in supergravity and D-brane models, Nucl. Phys. B 606 (2001) 59 hep-ph/0102181;

see also ref. [3]. 
[10] K.L. Chan, U. Chattopadhyay and P. Nath, Naturalness, weak scale supersymmetry and the prospect for the observation of supersymmetry at the Tevatron and at the LHC, Phys. Rev. D $\mathbf{5 8}$ (1998) 096004;

J.L. Feng, K.T. Matchev and T. Moroi, Multi-TeV scalars are natural in minimal supergravity, Phys. Rev. Lett. 84 (2000) 2322 hep-ph/9908309;

see also H. Baer, C.-h. Chen, F. Paige and X. Tata, Signals for minimal supergravity at the cern large hadron collider: multi - jet plus missing energy channel, Phys. Rev. D 52 (1995) 2746 hep-ph/9503271;

H. Baer, C.-h. Chen, M. Drees, F. Paige and X. Tata, Probing minimal supergravity at the cern LHC for large $\tan \beta$, Phys. Rev. D 59 (1999) 055014 hep-ph/9809223;

for a model-independent approach, see H. Baer, T. Krupovnickas, S. Profumo and P. Ullio, Model independent approach to focus point supersymmetry: from dark matter to collider searches, JHEP 10 (2005) 020 hep-ph/0507282.

[11] M. Drees and M.M. Nojiri, The neutralino relic density in minimal $N=1$ supergravity, Phys. Rev. D 47 (1993) 376 hep-ph/9207234;

H. Baer and M. Brhlik, Neutralino dark matter in minimal supergravity: direct detection vs. collider searches, Phys. Rev. D 57 (1998) 567 hep-ph/9706509;

$\mathrm{H}$. Baer et al., Yukawa unified supersymmetric $\mathrm{SO}(10)$ model: cosmology, rare decays and collider searches, Phys. Rev. D 63 (2001) 015007 hep-ph/0005027;

J.R. Ellis, T. Falk, G. Ganis, K.A. Olive and M. Srednicki, The CMSSM parameter space at large $\tan \beta$, Phys. Lett. B 510 (2001) 236 hep-ph/0102098;

L. Roszkowski, R. Ruiz de Austri and T. Nihei, New cosmological and experimental constraints on the CMSSM, JHEP 0108 (2001) 024 hep-ph/0106334;

A. Djouadi, M. Drees and J.L. Kneur, Constraints on the minimal supergravity model and prospects for SUSY particle production at future linear $e^{+} e^{-}$colliders, JHEP 08 (2001) 055 hep-ph/0107316;

A.B. Lahanas and V.C. Spanos, Implications of the pseudo-scalar Higgs boson in determining the neutralino dark matter, Eur. Phys. J. C 23 (2002) 185 hep-ph/0106345.

[12] P. Nath and R. Arnowitt, Predictions in $\mathrm{SU}(5)$ supergravity grand unification with proton stability and relic density constraints, Phys. Rev. Lett. 70 (1993) 3696 hep-ph/9302318;

H. Baer and M. Brhlik, ref. [8];

A. Djouadi, M. Drees and J.-L. Kneur, Neutralino dark matter in mSUGRA: reopening the light Higgs pole window, Phys. Lett. B 624 (2005) 60 hep-ph/0504090.

[13] C. Böhm, A. Djouadi and M. Drees, Light scalar top quarks and supersymmetric dark matter, Phys. Rev. D 62 (2000) 035012;

J.R. Ellis, K.A. Olive and Y. Santoso, Calculations of neutralino-stop coannihilation in the CMSSM, Astropart. Phys. 18 (2003) 395;

J. Edsjö et al., Accurate relic densities with neutralino, chargino and sfermion coannihilations in $M S U G R A$, JCAP 04 (2003) 001 hep-ph/0301106.

[14] H. Baer, A. Belyaev, T. Krupovnickas and A. Mustafayev, SUSY normal scalar mass hierarchy reconciles $(g-2)_{\mu}, b \rightarrow s \gamma$ and relic density, JHEP 06 (2004) 044 hep-ph/0403214.

[15] H. Baer, A. Mustafayev, S. Profumo, A. Belyaev and X. Tata, Direct, indirect and collider detection of neutralino dark matter in SUSY models with non-universal Higgs masses, JHEP 07 (2005) 065 hep-ph/0504001. 
[16] D. Auto, H. Baer, A. Belyaev and T. Krupovnickas, Reconciling neutralino relic density with yukawa unified supersymmetric models, JHEP 10 (2004) 066 hep-ph/0407165.

[17] S. Profumo, Neutralino dark matter, b- $\tau$ Yukawa unification and non- universal sfermion masses, Phys. Rev. D 68 (2003) 015006 hep-ph/0304071.

[18] J.R. Ellis, K.A. Olive and Y. Santoso, The MSSM parameter space with non-universal Higgs masses, Phys. Lett. B 539 (2002) 107 hep-ph/0204192;

J.R. Ellis, T. Falk, K.A. Olive and Y. Santoso, Exploration of the MSSM with non-universal Higgs masses, Nucl. Phys. B 652 (2003) 259 hep-ph/0210205.

[19] M. Drees, Supersymmetric dark matter 2004, hep-ph/0410113.

[20] A. Brignole, L.E. Ibáñez and C. Muñoz, Towards a theory of soft terms for the supersymmetric standard model, Nucl. Phys. B 422 (1994) 125 [hep-ph/9308271];

A. Brignole, L.E. Ibáñez, C. Muñoz and C. Scheich, Some issues in soft SUSY breaking terms from dilaton/moduli sectors, Z. Physik C 74 (1997) 157 hep-ph/9508258.

[21] C.H. Chen, M. Drees and J.F. Gunion, A non-standard string/SUSY scenario and its phenomenological implications, Phys. Rev. D 55 (1997) 330 hep-ph/9607421.

[22] R. Dermisek and A. Mafi, $\mathrm{SO}(10)$ grand unification in five dimensions: proton decay and the u problem, Phys. Rev. D 65 (2002) 055002 hep-ph/0108139;

$\mathrm{H}$. Baer et al., Viable models with non-universal gaugino mediated supersymmetry breaking, JHEP 05 (2002) 061 hep-ph/0204108;

C. Balazs and R. Dermisek, Yukawa coupling unification and non-universal gaugino mediation of supersymmetry breaking, JHEP 06 (2003) 024 hep-ph/0303161.

[23] H. Baer, M.A. Diaz, P. Quintana and X. Tata, Impact of physical principles at very high energy scales on the superparticle mass spectrum, JHEP 04 (2000) 016 hep-ph/0002245.

[24] H. Baer, A. Mustafayev, E.-K. Park and S. Profumo, Mixed wino dark matter: consequences for direct, indirect and collider detection, JHEP 07 (2005) 046 hep-ph/0505227.

[25] L. Randall and R. Sundrum, Out of this world supersymmetry breaking, Nucl. Phys. B 557 (1999) 79 hep-th/9810155;

G. Giudice, M. Luty, H. Murayama and R. Rattazzi, Gaugino mass without singlets, JHEP $9812(1998) 27$ hep-ph/9810442.

[26] J. Amundson et al., Report of the supersymmetry theory subgroup, ECONF C960625 (1996) SUP106 hep-ph/9609374];

G. Anderson, H. Baer, C.-h. Chen and X. Tata, The reach of Fermilab Tevatron upgrades for $\mathrm{SU}(5)$ supergravity models with non-universal gaugino masses, Phys. Rev. D 61 (2000) 095005 hep-ph/9903370.

[27] N. Chamoun, C.-S. Huang, C. Liu and X.-H. Wu, Non-universal gaugino masses in supersymmetric SO(10), Nucl. Phys. B 624 (2002) 81 hep-ph/0110332.

[28] U. Chattopadhyay, A. Corsetti and P. Nath, Supersymmetric dark matter and Yukawa unification, Phys. Rev. D 66 (2002) 035003 hep-ph/0201001.

[29] K. Griest and L. Roszkowski, Effect of relaxing grand unification assumptions on neutralinos in the minimal supersymmetric model, Phys. Rev. D 46 (1992) 3309.

[30] T. Moroi and L. Randall, Wino cold dark matter from anomaly-mediated SUSY breaking, Nucl. Phys. B 570 (2000) 455 hep-ph/9906527. 
[31] A. Corsetti and P. Nath, Gaugino mass nonuniversality and dark matter in sugra, strings and D-brane models, Phys. Rev. D 64 (2001) 125010 hep-ph/0003186.

[32] A. Birkedal-Hansen and B.D. Nelson, The role of wino content in neutralino dark matter, Phys. Rev. D 64 (2001) 015008 hep-ph/0102075]; Relic neutralino densities and detection rates with nonuniversal gaugino masses, Phys. Rev. D 67 (2003) 095006 hep-ph/0211071.

[33] V. Bertin, E. Nezri and J. Orloff, Neutralino dark matter beyond CMSSM universality, JHEP $02(2003) 046$ hep-ph/0210034.

[34] A. Bottino, F. Donato, N. Fornengo and S. Scopel, Indirect signals from light neutralinos in supersymmetric models without gaugino mass unification, Phys. Rev. D 70 (2004) 015005 hep-ph/0401186.

[35] G. Belanger, F. Boudjema, A. Cottrant, A. Pukhov and A. Semenov, WMAP constraints on sugra models with non-universal gaugino masses and prospects for direct detection, Nucl. Phys. B 706 (2005) 411 hep-ph/0407218.

[36] D.G. Cerdeno and C. Muñoz, Neutralino dark matter in supergravity theories with nonuniversal scalar and gaugino masses, JHEP 10 (2004) 015 hep-ph/0405057;

Y. Mambrini and E. Nezri, Dark matter and colliders searches in the MSSM, hep-ph/0507263;

see also S. Baek, D.G. Cerdeno, Y.G. Kim, P. Ko and C. Muñoz, Direct detection of neutralino dark matter in supergravity, JHEP 06 (2005) 017 hep-ph/0505019.

[37] A. Masiero, S. Profumo and P. Ullio, Neutralino dark matter detection in split supersymmetry scenarios, Nucl. Phys. B 712 (2005) 86 hep-ph/0412058].

[38] H. Baer and X. Tata, Weak scale supersymmetry: from superfields to scattering events, Cambridge University Press, Cambridge 2006.

[39] F.E. Paige, S.D. Protopescu, H. Baer and X. Tata, Isajet 7.69: a monte carlo event generator for $p p, \bar{p} p$ and $e^{+} e^{-}$reactions, hep-ph/0312045.

[40] CompHEP collaboration, E. Boos et al., CompHEP 4.4 - Automatic computations from lagrangians to events, Nucl. Instrum. Meth. A534 (2004) 250.

[41] P. Gondolo and G. Gelmini, Cosmic abundances of stable particles: improved analysis, Nucl. Phys. B 360 (1991) 145;

J. Edsjo and P. Gondolo, Neutralino relic density including coannihilations, Phys. Rev. D 56 (1997) 1879 hep-ph/9704361.

[42] H. Baer, V.D. Barger, D. Karatas and X. Tata, Detecting gluinos at hadron supercolliders, Phys. Rev. D 36 (1987) 96.

[43] K. Griest and D. Seckel, Three exceptions in the calculation of relic abundances, Phys. Rev. D 43 (1991) 3191.

[44] J.L. Feng, T. Moroi, L. Randall, M. Strassler and S.-f. Su, Discovering supersymmetry at the Tevatron in wino LSP scenarios, Phys. Rev. Lett. 83 (1999) 1731 hep-ph/9904250.

[45] J. Edsjo, M. Schelke, P. Ullio and P. Gondolo, Accurate relic densities with neutralino, chargino and sfermion coannihilations in mSUGRA, JCAP 04 (2003) 001 hep-ph/0301106.

[46] S. Profumo, $\mathrm{TeV} \gamma$-rays and the largest masses and annihilation cross sections of neutralino dark matter, astro-ph/0508628. 
[47] S. Profumo and C.E. Yaguna, Gluino coannihilations and heavy bino dark matter, Phys. Rev. D 69 (2004) 115009 hep-ph/0402208.

[48] For a review, see e.g. G. Eigen, R. Gaitskell, G.D. Kribs and K.T. Matchev, Indirect investigations of supersymmetry, eConf C010630 (2001) P342 hep-ph/0112312;

see also D. Hooper and L.-T. Wang, Direct and indirect detection of neutralino dark matter in selected supersymmetry breaking scenarios, Phys. Rev. D 69 (2004) 035001 hep-ph/0309036;

W. de Boer, M. Herold, C. Sander and V. Zhukov, Indirect evidence for the supersymmetric nature of dark matter from the combined data on galactic positrons, antiprotons and gamma rays, hep-ph/0309029.

[49] P. Gondolo et al., Darksusy: a numerical package for supersymmetric dark matter calculations, astro-ph/0211238.

[50] J.F. Navarro et al., The inner structure of lambdacdm halos III: universality and asymptotic slopes, Mon. Not. Roy. Astron. Soc. 349 (2004) 1039 astro-ph/0311231;

the adiabatic contraction of the halo follows G.R. Blumenthal, S.M. Faber, R. Flores and J.R. Primack, Contraction of dark matter galactic halos due to baryonic infall, Astrophys. J. 301 (1986) 27;

for the halo parameter choices see also ref. [53].

[51] H. Baer and J.O'Farrill, Probing neutralino resonance annihilation via indirect detection of dark matter, JCAP 04 (2004) 005.

[52] H. Baer and J. O'Farrill, Probing neutralino resonance annihilation via indirect detection of dark matter, JCAP 04 (2004) 005 hep-ph/0312350.

[53] S. Profumo and P. Ullio, The role of antimatter searches in the hunt for supersymmetric dark matter, JCAP 07 (2004) 006 hep-ph/0406018.

[54] For a recent analysis, see H. Baer, C. Balazs, A. Belyaev and J.O'Farrill, Direct detection of dark matter in supersymmetric models, JCAP 0309 (2003) 007;

a subset of earlier work includes M. Goodman and E. Witten, Detectability of certain dark matter candidates, Phys. Rev. D 31 (1985) 3059;

M.W. Goodman and E. Witten, Detectability of certain dark-matter candidates, Phys. Rev. D 31 (1985) 3059;

K. Griest, Calculations of rates for direct detection of neutralino dark matter, Phys. Rev. Lett. 61 (1988) 666; Phys. Rev. D 38 (1988) 2357;

M. Drees and M.M. Nojiri, New contributions to coherent neutralino-nucleus scattering, Phys. Rev. D 47 (1993) 4226 hep-ph/9210272; ; Phys. Rev. D 48 (1993) 3483;

V.A. Bednyakov, H.V. Klapdor-Kleingrothaus and S. Kovalenko, On SUSY dark matter detection with spinless nuclei, Phys. Rev. D 50 (1994) 7128 hep-ph/9401262;

P. Nath and R. Arnowitt, Event rates in dark matter detectors for neutralinos including constraints from theb $\rightarrow$ s $\gamma$ decay, Phys. Rev. Lett. 74 (1995) 4592 hep-ph/9409301;

L. Bergstrom and P. Gondolo, Astropart. Phys. 5 (1996) 263;

H. Baer and M. Brhlik, Neutralino dark matter in minimal supergravity: direct detection vs. collider searches, Phys. Rev. D 57 (1998) 567 hep-ph/9706509;

J.R. Ellis, A. Ferstl and K.A. Olive, Re-evaluation of the elastic scattering of supersymmetric dark matter, Phys. Lett. B 481 (2000) 304 hep-ph/0001005; Phys. Rev. D 63 (2001) 065016 :

E. Accomando, R. Arnowitt, B. Dutta and Y. Santoso, Neutralino proton cross sections in supergravity models, Nucl. Phys. B 585 (2000) 124 hep-ph/0001019; 
A. Bottino, F. Donato, N. Fornengo and S. Scopel, Probing the supersymmetric parameter space by WIMP direct detection, Phys. Rev. D 63 (2001) 125003 hep-ph/0010203;

M.E. Gomez and J.D. Vergados, Cold dark matter detection in SUSY models at large $\tan \beta$, Phys. Lett. B 512 (2001) 252 hep-ph/0012020;

A.B. Lahanas, D.V. Nanopoulos and V.C. Spanos, Dark matter direct searches and the anomalous magnetic moment of muon, Phys. Lett. B 518 (2001) 94 hep-ph/0107151;

A. Corsetti and P. Nath, Phys. Rev. D 64 (2001) 115009;

S. Bergmann and G. Perez, Constraining models of new physics in light of recent experimental results on a (psi k(s)), Phys. Rev. D 64 (2001) 115009 hep-ph/0103299;;

E.A. Baltz and P. Gondolo, Implications of muon anomalous magnetic moment for supersymmetric dark matter, Phys. Rev. Lett. 86 (2001) 5004 hep-ph/0102147;;

M. Drees, Y.G. Kim, T. Kobayashi and M.M. Nojiri, Direct detection of neutralino dark matter and the anomalous dipole moment of the muon, Phys. Rev. D 63 (2001) 115009 hep-ph/0011359;

see also J.L. Feng, K.T. Matchev and F. Wilczek, Neutralino dark matter in focus point supersymmetry, Phys. Lett. B 482 (2000) 388 hep-ph/0004043;

J.R. Ellis, A. Ferstl, K.A. Olive and Y. Santoso, Direct detection of dark matter in the MSSM with non- universal Higgs masses, Phys. Rev. D 67 (2003) 123502 hep-ph/0302032;

J.R. Ellis, K.A. Olive, Y. Santoso and V.C. Spanos, High-energy constraints on the direct detection of MSSM neutralinos, Phys. Rev. D 69 (2004) 015005 hep-ph/0308075;

see C. Muñoz, Dark matter detection in the light of recent experimental results, Int. J. Mod. Phys. A 19 (2004) 3093 hep-ph/0309346 for a recent review.

[55] See H. Baer, C. Balazs, A. Belyaev and J. O'Farrill, ref. 54].

[56] CDMS collaboration, D.S. Akerib et al., First results from the cryogenic dark matter search in the soudan underground lab, Phys. Rev. Lett. 93 (2004) 211301 astro-ph/0405033.

[57] A. Benoit et al., Improved exclusion limits from the edelweiss WIMP search, Phys. Lett. B 545 (2002) 43 astro-ph/0206271.

[58] CRESST collaboration, M. Bravin et al., The CRESST dark matter search, Astrophys. J. 12 (1999) 107 .

[59] ZePLIN-1 collaboration, N. Spooner et al., The UKDM single phase xenon experiment at boulby, in Proc. of the APS/DPF/DPB Summer Study on the Future of Particle Physics (Snowmass 2001), ed. N. Graf, eConf C010630, E(2001) 601.

[60] Xenon collaboration, Y. Suzuki, Low energy solar neutrino detection by using liquid xenon, hep-ph/0008296.

[61] I. Abt et al., A new ge-76 double $\beta$ decay experiment at LNGS, hep-ex/0404039.

[62] D.B. Cline et al., Status of Zeplin II and Zeplin IV study, Nucl. Phys. 124 (Proc. Suppl.) (2003) 229.

[63] See talk by C. Rubbia at 6th UCLA Symposium on Sources and detection of dark matter and dark energy in the universe, Marina del Ray, CA, February 2004.

[64] J. Silk, K.A. Olive and M. Srednicki, The photino, the sun and high-energy neutrinos, Phys. Rev. Lett. 55 (1985) 257;

K. Freese, Can scalar neutrinos or massive dirac neutrinos be the missing mass?, Phys. Lett. B 167 (1986) 295; 
L.M. Krauss, M. Srednicki and F. Wilczek, Solar system constraints and signatures for dark matter candidates, Phys. Rev. D 33 (1986) 2079;

V. Berezinsky, A. Bottino, J. R. Ellis, N. Fornengo, G. Mignola and S. Scopel, Searching for relic neutralinos using neutrino telescopes, Astropart. Phys. 5 (1996) 333;

L. Bergstrom, J. Edsjo and P. Gondolo, Indirect neutralino detection rates in neutrino telescopes, Phys. Rev. D 55 (1997) 1765 hep-ph/9607237;

A. Bottino, F. Donato, N. Fornengo and S. Scopel, Combining the data of annual modulation effect in WIMP direct detection with measurements of WIMP indirect searches, Astropart. Phys. 10 (1999) 203 hep-ph/9809239;

A. Corsetti and P. Nath, Out-going muon flux from neutralino annihilation in the sun and the earth in supergravity unification, Int. J. Mod. Phys. A 15 (2000) 905 hep-ph/9904497; V.D. Barger, F. Halzen, D. Hooper and C. Kao, Indirect search for neutralino dark matter with high energy neutrinos, Phys. Rev. D 65 (2002) 075022 hep-ph/0105182;

V. Bertin, E. Nezri and J. Orloff, Neutrino indirect detection of neutralino dark matter in the CMSSM, Eur. Phys. J. C 26 (2002) 111 [hep-ph/0204135; Neutralino dark matter beyond CMSSM universality, JHEP 0302 (2003) 046.

[65] ANTARES collaboration, E. Carmona, Status report of the antares neutrino telescope, Nucl. Phys. 95 (Proc. Suppl.) (2001) 161.

[66] The IceCuBe collaboration, J. Ahrens et al., Icecube: the next generation neutrino telescope at the south pole, Nucl. Phys. 118 (Proc. Suppl.) (2003) 388 astro-ph/0209556;

F. Halzen, High-energy neutrino astronomy: from Amanda to Icecube, astro-ph/0311004;

F. Halzen and D. Hooper, Icecube-plus: an ultra-high energy neutrino telescope, JCAP 01 (2004) 002 astro-ph/0310152.

[67] F.W. Stecker, $\gamma$-ray constraints on dark matter reconsidered, Phys. Lett. B 201 (1988) 529; F.W. Stecker and A.J. Tylka, Spectra, fluxes and observability of $\gamma$-rays from dark matter annihilation in the galaxy, Astrophys. J. 343 (1989) 169;

S. Rudaz and F.W. Stecker, On the observability of the $\gamma$-ray line flux from dark matter annihilation, Astrophys. J. 368 (1991) 406;

M. Urban et al., Searching for TeV dark matter by atmospheric Cherenkov techniques, Phys. Lett. B 293 (1992) 149 hep-ph/9208255;

V.S. Berezinsky, A.V. Gurevich and K.P. Zybin, Distribution of dark matter in the galaxy and the lower limits for the masses of supersymmetric particles, Phys. Lett. B 294 (1992) 221; V. Berezinsky, A. Bottino and G. Mignola, High-energy $\gamma$ radiation from the galactic center due to neutralino annihilation, Phys. Lett. B 325 (1994) 136 hep-ph/9402215;

L. Bergstrom, P. Ullio and J. H. Buckley, Observability of $\gamma$-rays from dark matter neutralino annihilations in the milky way halo, Astropart. Phys. 9 (1998) 137.

L. Bergstrom, J. Edsjo and P. Ullio, Possible indications of a clumpy dark matter halo, Phys. Rev. D 58 (1998) 083507 astro-ph/9804050;

J. Buckley et al., $\Gamma$-ray summary report, astro-ph/0201160;

P. Ullio, L. Bergstrom, J. Edsjo and C.G. Lacey, Cosmological dark matter annihilations into

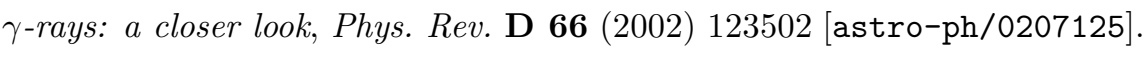

[68] EGRET collaboration, H. A. Mayer-Hasselwander et al., High-energy $\gamma$-ray emission from the galactic center, MPE (1998) 440.

[69] GLAST collaboration, A. Morselli, A. Lionetto, A. Cesarini, F. Fucito and P. Ullio, Search for dark matter with glast, Nucl. Phys. 113 (Proc. Suppl.) (2002) 213 astro-ph/0211327. 
[70] S. Rudaz and F.W. Stecker, Cosmic ray anti-protons, positrons and $\gamma$-rays from halo dark matter annihilation, Astrophys. J. 325 (1988) 16:

A.J. Tylka, Cosmic ray positrons from annihilation of weakly interacting massive particles in the galaxy, Phys. Rev. Lett. 63 (1989) 840;

M.S. Turner and F. Wilczek, Positron line radiation from halo WIMP annihilations as a dark matter signature, Phys. Rev. D 42 (1990) 1001;

M. Kamionkowski and M.S. Turner, A distinctive positron feature from heavy WIMP annihilations in the galactic halo, Phys. Rev. D 43 (1991) 1774;

I.V. Moskalenko and A.W. Strong, Positrons from particle dark-matter annihilation in the galactic halo: propagation Green's functions, Phys. Rev. D 60 (1999) 063003 astro-ph/9905283;

E.A. Baltz and J. Edsjo, Positron propagation and fluxes from neutralino annihilation in the halo, Phys. Rev. D 59 (1999) 023511; astro-ph/9808243;

G.L. Kane, L.-T. Wang and J.D. Wells, Supersymmetry and the positron excess in cosmic rays, Phys. Rev. D 65 (2002) 057701 hep-ph/0108138;

E.A. Baltz, J. Edsjo, K. Freese and P. Gondolo, The cosmic ray positron excess and neutralino dark matter, Phys. Rev. D 65 (2002) 063511 astro-ph/0109318;

G.L. Kane, L.-T. Wang and T.T. Wang, Supersymmetry and the cosmic ray positron excess, Phys. Lett. B 536 (2002) 263 hep-ph/0202156;

D. Hooper, J.E. Taylor and J. Silk, Can supersymmetry naturally explain the positron excess?, Phys. Rev. D 69 (2004) 103509 hep-ph/0312076.

[71] M.A. DuVernois et al., Cosmic ray electrons and positrons from 1-GeV to 100-GeV: measurements with heat and their interpretation, Astrophys. J. 559 (2001) 296.

[72] PAMELA collaboration, M. Pearce, The status of the Pamela experiment, Nucl. Phys. 113 (Proc. Suppl.) (2002) 314.

[73] AMS collaboration, J. Casaus, The ams experiment: a magnetic spectrometer in space, Nucl. Phys. 114 (Proc. Suppl.) (2003) 259.

[74] F.W. Stecker, S. Rudaz and T.F. Walsh, Galactic anti-protons from photinos, Phys. Rev. Lett. 55 (1985) 2622;

F. Stecker and A. J. Tylka, The cosmic ray anti-proton spectrum from dark matter annihilation and its astrophysical implications: a new look, Astrophys. J. 336 (1989) L51; P. Chardonnet, G. Mignola, P. Salati and R. Taillet, Galactic diffusion and the antiproton signal of supersymmetric dark matter, Phys. Lett. B 384 (1996) 161 astro-ph/9606174;

A. Bottino, F. Donato, N. Fornengo and P. Salati, Which fraction of the measured cosmic ray antiprotons might be due to neutralino annihilation in the galactic halo?, Phys. Rev. D 58 (1998) 123503 astro-ph/9804137;

L. Bergstrom, J. Edsjo and P. Ullio, Cosmic anti-protons as a probe for supersymmetric dark matter?, Astrophys. J. 526 (1999) 215 astro-ph/9902012.

[75] BESS collaboration, S. Orito et al., Precision measurement of cosmic-ray antiproton spectrum, Phys. Rev. Lett. 84 (2000) 1078 astro-ph/9906426.

[76] H. Fuke et al., Search for cosmic-ray antideuterons, Phys. Rev. Lett. 95 (2005) 081101 astro-ph/0504361.

[77] K. Mori et al., A novel antimatter detector based on $x$-ray deexcitation of exotic atoms, Astrophys. J. 566 (2002) 604 astro-ph/0109463. 
[78] S. Profumo and C.E. Yaguna, A statistical analysis of supersymmetric dark matter in the MSSM after WMAP, Phys. Rev. D 70 (2004) 095004 hep-ph/0407036.

[79] H. Baer and S. Profumo, Low energy antideuterons: shedding light on dark matter, astro-ph/0510722.

[80] H. Komatsu and H. Kubo, The radiative photino decay into a higgsino, Phys. Lett. B 157 (1985) 90; Decay of the light neutralino, Nucl. Phys. B 263 (1986) 265;

H.E. Haber, G. Kane and M. Quiros, Is low-energy supergravity consistent with cosmology and particle physics experiments?, Phys. Lett. B 160 (1985) 297 and A nonminimal supergravity model consistent with all experimental constraints, Nucl. Phys. B 273 (1986) 333;

M. Quiros, G.L. Kane and H.E. Haber, A nonminimal supergravity model consistent with all experimental constraints, Nucl. Phys. B 273 (1986) 333;

G. Gamberini, Heavy gluino and squark decays at pp̄ collider, Z. Physik C 30 (1986) 605;

R. Barbieri, G. Gamberini, G.F. Giudice and G. Ridolfi, Signals of supersymmetry at the $Z^{0}$ resonance, Nucl. Phys. B 296 (1988) 75.

[81] H.E. Haber and D. Wyler, Radiative neutralino decay, Nucl. Phys. B 323 (1989) 267.

[82] S. Ambrosanio and B. Mele, Neutralino decays in the minimal supersymmetric standard model, Phys. Rev. D 53 (1996) 2541 hep-ph/9508237.

[83] H. Baer and T. Krupovnickas, Radiative neutralino decay in supersymmetric models, JHEP 09 (2002) 038 hep-ph/0208277.

[84] H. Baer, J.R. Ellis, G.B. Gelmini, D.V. Nanopoulos and X. Tata, Squark decays into gauginos at the p $\bar{p}$ collider, Phys. Lett. B 161 (1985) 175;

G. Gamberini, Heavy gluino and squark decays at pp collider, Z. Physik C 30 (1986) 605;

H. Baer, V.D. Barger, D. Karatas and X. Tata, Detecting gluinos at hadron supercolliders, Phys. Rev. D 36 (1987) 96;

H. Baer, X. Tata and J. Woodside, Multi - lepton signals from supersymmetry at hadron super colliders, Phys. Rev. D 45 (1992) 142.

[85] H. Baer, K. Hagiwara and X. Tata, Gauginos as a signal for supersymmetry at $p \bar{p}$ colliders, Phys. Rev. D 35 (1987) 1598; Gluino and squark production in association with gauginos at hadron supercolliders, Phys. Rev. D 42 (1990) 2259;

H. Baer, C. Kao and X. Tata, Aspects of chargino - neutralino production at the Tevatron collider, Phys. Rev. D 48 (1993) 5175 hep-ph/9307347;

H. Baer, C.-h. Chen, F. Paige and X. Tata, Trileptons from chargino - neutralino production at the cern large hadron collider, Phys. Rev. D 50 (1994) 4508 hep-ph/9404212.

[86] I. Hinchliffe, F.E. Paige, M.D. Shapiro, J. Soderqvist and W. Yao, Precision SUSY measurements at LHC, Phys. Rev. D 55 (1997) 5520 hep-ph/9610544;

H. Bachacou, I. Hinchliffe and F.E. Paige, Measurements of masses in sugra models at LHC, Phys. Rev. D 62 (2000) 015009 [hep-ph/9907518];

ATLAS collaboration, Atlas detector and physics performance: technical design report, LHCC 99-14/15.

[87] D.A. Dicus, S. Nandi and X. Tata, W decay in supergravity guts, Phys. Lett. B 129 (1983) 451;

A.H. Chamseddine, P. Nath and R. Arnowitt, Experimental signals for supersymmetric decays of the $W$ and $Z$ bosons, Phys. Lett. B 129 (1983) 445;

$\mathrm{H}$. Baer and X. Tata, Multi - lepton signals from w+- and z0 decays to gauginos at $\bar{p} p$ colliders, Phys. Lett. B 155 (1985) 278; 


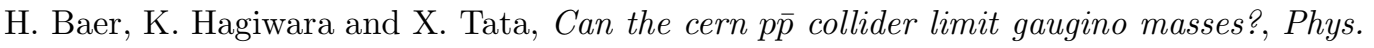
Rev. Lett. 57 (1986) 294;

R. Arnowitt and P. Nath, Supersymmetric signals at the Tevatron, Mod. Phys. Lett. A 2 (1987) 331;

R. Barbieri, F. Caravaglios, M. Frigeni and M.L. Mangano, Production and leptonic decays of charginos and neutralinos in hadronic collisions, Nucl. Phys. B 367 (1991) 28;

$\mathrm{H}$. Baer and X. Tata, Probing charginos and neutralinos beyond the reach of lep at the Tevatron collider, Phys. Rev. D 47 (1993) 2739;

J.L. Lopez, D.V. Nanopoulos, X. Wang and A. Zichichi, Supersymmetry tests at Fermilab: a proposal, Phys. Rev. D 48 (1993) 2062 hep-ph/9211286;

H. Baer, C. Kao and X. Tata, Aspects of chargino - neutralino production at the Tevatron collider, Phys. Rev. D 48 (1993) 5175 hep-ph/9307347;

S. Mrenna, G.L. Kane, G.D. Kribs and J.D. Wells, Possible signals of constrained minimal supersymmetry at a high luminosity Fermilab Tevatron collider, Phys. Rev. D 53 (1996) 1168 hep-ph/9505245;

H. Baer, C.-h. Chen, F. Paige and X. Tata, Supersymmetry reach of Tevatron upgrades: a comparative study, Phys. Rev. D 54 (1996) 5866 hep-ph/9604406;

V.D. Barger, C. Kao and T.-j. Li, Trilepton signal of minimal supergravity at the Tevatron including tau-lepton contributions, Phys. Lett. B 433 (1998) 328 hep-ph/9804451;

K.T. Matchev and D.M. Pierce, Supersymmetry reach of the Tevatron via trilepton, like- sign dilepton and dilepton plus $\tau$ jet signatures, Phys. Rev. D 60 (1999) 075004 hep-ph/9904282;

H. Baer, C.-h. Chen, M. Drees, F. Paige and X. Tata, Collider phenomenology for supersymmetry with large $\tan \beta$, Phys. Rev. Lett. 79 (1997) 986 hep-ph/9704457;

J.D. Lykken and K.T. Matchev, Supersymmetry signatures with $\tau$ jets at the Tevatron, Phys. Rev. D 61 (2000) 015001 hep-ph/9903238.

[88] H. Baer, M. Drees, F. Paige, P. Quintana and X. Tata, Trilepton signal for supersymmetry at the Fermilab Tevatron revisited, Phys. Rev. D 61 (2000) 095007 hep-ph/9906233;

V.D. Barger and C. Kao, Trilepton signature of minimal supergravity at the upgraded Tevatron, Phys. Rev. D 60 (1999) 115015 hep-ph/9811489;

K.T. Matchev and D.M. Pierce, New backgrounds in trilepton, dilepton and dilepton plus tau jet SUSY signals at the Tevatron, Phys. Lett. B 467 (1999) 225 hep-ph/9907505;

H. Baer, T. Krupovnickas and X. Tata, Reach of the fermilab Tevatron for minimal supergravity in the region of large scalar masses, JHEP 07 (2003) 020 hep-ph/0305325.

[89] H. Baer, J.R. Ellis, G.B. Gelmini, D.V. Nanopoulos and X. Tata, Squark decays into gauginos at the $p \bar{p}$ collider, Phys. Lett. B 161 (1985) 175;

G. Gamberini, Heavy gluino and squark decays at pp collider, Z. Physik C 30 (1986) 605;

H. Baer, X. Tata and J. Woodside, Multi - lepton signals from supersymmetry at hadron super colliders, Phys. Rev. D 45 (1992) 142.

[90] H. Baer, C. Balazs, A. Belyaev, T. Krupovnickas and X. Tata, Updated reach of the cern LHC and constraints from relic density, $b \rightarrow s \gamma$ and $A(\mu)$ in the mSUGRA model, JHEP 06 (2003) 054 hep-ph/0304303;

For earlier work, see H. Baer, C. H. Chen, F. Paige and X. Tata, Signals for minimal supergravity at the CERN large hadron collider: multi - Jet plus missing energy channel, Phys. Rev. D 52 (1995) 2746 hep-ph/9503271]; Signals for minimal supergravity at the CERN large hadron collider. 2: multi - lepton channels, Phys. Rev. D 53 (1996) 6241 hep-ph/9512383; 
H. Baer, C.-h. Chen, M. Drees, F. Paige and X. Tata, Probing minimal supergravity at the cern LHC for large $\tan \beta$, Phys. Rev. D 59 (1999) 055014 hep-ph/9809223;

S. Abdullin and F. Charles, Search for SUSY in (Leptons +) Jets $+E_{T}^{\text {miss }}$ final states, Nucl. Phys. B 547 (1999) 60 hep-ph/9811402;

CMS collaboration, S. Abdullin et al., Discovery potential for supersymmetry in CMS, J. Phys. G 28 (2002) 469 hep-ph/9806366;

B.C. Allanach, J.P.J. Hetherington, M.A. Parker and B.R. Webber, Naturalness reach of the large hadron collider in minimal supergravity, JHEP 08 (2000) 017 hep-ph/0005186.

[91] H. Baer, C. Balazs, A. Belyaev, T. Krupovnickas and X. Tata, Updated reach of the cern LHC and constraints from relic density, $b \rightarrow s \gamma$ and $A(\mu)$ in the mSUGRA model, JHEP 06 (2003) 054 hep-ph/0304303.

[92] H. Baer, A. Belyaev, T. Krupovnickas and X. Tata, Linear collider capabilities for supersymmetry in dark matter allowed regions of the mSUGRA model, JHEP 02 (2004) 007 hep-ph/0311351;

H. Baer, T. Krupovnickas and X. Tata, Two photon background and the reach of a linear collider for supersymmetry in WMAP favored coannihilation regions, JHEP 06 (2004) 061 hep-ph/0405058.

[93] T. Tsukamoto, K. Fujii, H. Murayama, M. Yamaguchi and Y. Okada, Precision study of supersymmetry at future linear $e+e-$ colliders, Phys. Rev. D 51 (1995) 3153.

[94] H. Baer, R. Munroe and X. Tata, Supersymmetry studies at future linear $e^{+} e^{-}$colliders, Phys. Rev. D 54 (1996) 6735 hep-ph/9606325.

[95] H. Baer, A. Bartl, D. Karatas, W. Majerotto and X. Tata, Searching for supersymmetry at $e^{+} e^{-}$supercolliders, Int. J. Mod. Phys. A 4 (1989) 4111. 